A Technique for Measurement of Vector and Tensor Polarization in Solid Spin One Polarized Targets

William Frederick Kielhorn

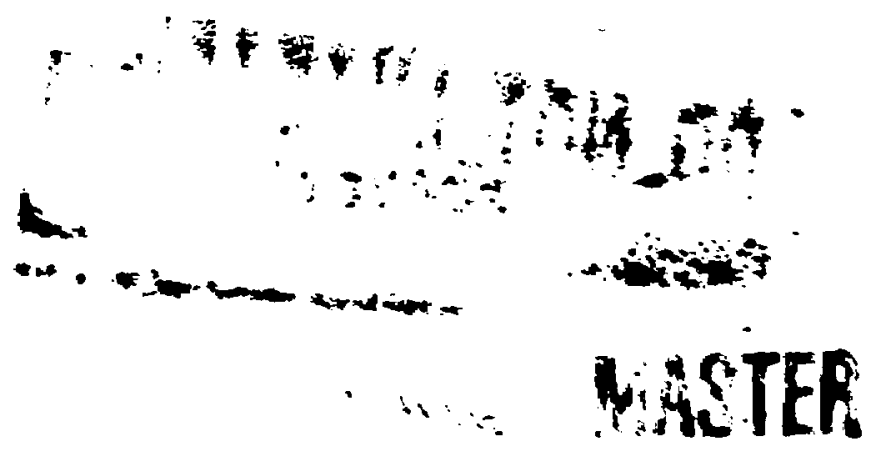

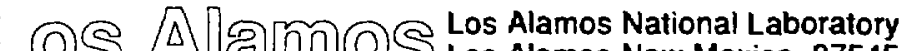

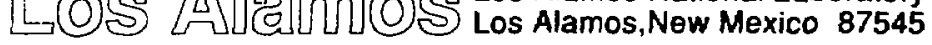




\section{Acknowledgments}

Foremost I want to thank Seppo Penttilä for numerous discussions and advice, relating to all matters ranging from physics to the proper spelling of his Finnish name. He tirelessly proofed the numerous drafts required to produce this document, and also provided a comfortable office in which to work. I would also like to thank Jerry Hoffmann for arranging financial support and exercising unsurpassed skill at bending the rules (and other people's arms). George Igo and Peter Riley suggested the problem, and I thank them. Peter arranged for one year's financial support. Finally, I thank Dick Werbeck, John Jarmer, Alma Sondreal and the entire MP-7 staff for support and encouragement, and Dick Boudrie for his help in arranging supplemental financial support.

William Frederick Kielhorn

The University of Texas at Austin

May, 1991 


\title{
A TECHNIQUE FOR MEASUREMENT OF VECTOR AND TENSOR POLARIZATION \\ IN SOLID SPIN ONE POLARIZED \\ TARGETS
}

\author{
by
}

William Frederick Kielhorn

\begin{abstract}
Vector and tensor polarizations are explicitly defined and used to characterize the polarization states of spin one polarized targets, and a technique for extracting these polarizations from nuclear magnetic resonance (NMR) data is developed. This technique is independent of assumptions about spin temperature, but assumes the target's crystal structure induces a quadrupole interaction with the spin one particles.

Analysis of the NMR signals involves a computer curve fitting algorithm inplemented with a fast Fourier transform method which speeds and simplifies curve fitting algorithms used previously. For accurate curve fitting, the NMR electronic circuit must be modeled by the fitting algorithm. Details of a circuit, its model, and data collected from this circuit are given for a solid deuterated ammonia target.
\end{abstract}




\section{Contents}

1 Introduction 1

2 Spin States for Spin One Targets 4

3 Absorption Line-shape for Spin One 19

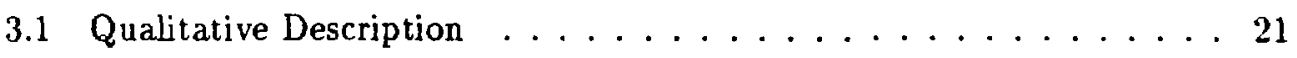

3.2 Mathematical Details . . . . . . . . . . . . . . . 24

4 Polarization Measurement for Spin One 45

4.1 Thermal Equilibrium Method . . . . . . . . . . . . . 49

4.2 Spin Temperature Method . . . . . . . . . . . . 50

4.3 Numerical Methods . . . . . . . . . . . . . . . 52

4.4 Circuit Model . . . . . . . . . . . . . . 55

4.5 Approximations . . . . . . . . . . . . . 62

4.6 Homodyne Detector . . . . . . . . . . . . . . 64

4.7 Computer Algorithm . . . . . . . . . . . . 68

5 Data $\quad 71$

$\begin{array}{ll}\text { A Kramers-Krönig Relation } & 85\end{array}$

B Fourier Transform Conventions and Results 93 
C $f_{\eta}$ : Derivation and Properties $\quad 95$

D Preparation of Target Material $\quad 110$ 


\section{List of Tables}

$3.1 \quad$ Non-zero diagonal elements of $\hat{Q}_{\alpha \beta} \ldots \ldots \ldots \ldots \ldots \ldots$

4.1 Cable parameters at various frequencies and temperatures. . . . 57

5.1 Material parameters for solid $\mathrm{ND}_{3} \ldots \ldots \ldots \ldots \ldots \ldots$ 


\section{List of Figures}

2.1 Basis matrices for density operator. . . . . . . . . 7

2.2 Allowed values of alignment, $A$, and vector polarization, $P$, for a cylindrically symmetric system lie on and inside the triangle. Values lying on the curve can be described by an effective temperature which is described in the text. Pure states lie at the vertices. . . . . . . 12

2.3 Polarization, alignment, effective temperature vs the population ratio. 16

3.1 Typical powder patterns showing definitions of $h_{1}, h_{2}, \Delta \nu_{p}$, and $\Delta \nu_{s}$. Note that $h_{1}$ and $h_{2}$ are negative when they extend below the zero axis. The dotted line indicates zero. . . . . . . . . . . 23

3.2 a) Energy level diagram for $\mathcal{H}_{Z e e}$ for $B_{Z e e}=25.00 \mathrm{KG}$. b) Schematic representation of corresponding line-shape (i.e., a delta function depicted here as a sharp peak). . . . . . . . . . . 27

3.3 a) Level shifts due to $H_{\text {quad }}$ b) Corresponding line-shape. . . . . . 30

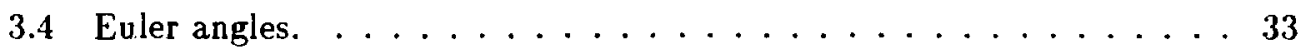

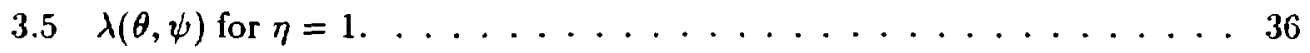

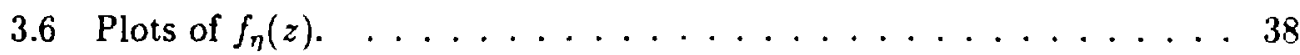

4.1 Schematic representation of connection to NMR coil. . . . . . . 56

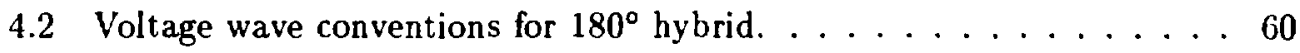

4.3 Homodyne detector. . . . . . . . . . . . . . . . 65

4.4 Schematic of complete detection circuit. . . . . . . . . 67

5.1 Typical signal and baseline data. . . . . . . . . it 
5.2 Typical signal data (solid) and corresponding model curve (dotted). The fitted curve has $r^{\prime}=1.674(P \approx 33 \%) . \ldots \ldots \ldots \ldots$

5.3 Two model curves are shown which differ only in that $r^{\prime}=1.698$ in one and $r^{\prime}=1.529$ in the other. From this we estimate how well the fitting procedure determines $r^{\prime}$. (See the text). . . . . . is

5.4 Typical $N D_{3}$ thermal equilibrium signal, with baseline subtracted. Temperature and field values were $1.016 \mathrm{~K}$ and $25.00 \mathrm{KG}$ respectively. At thermal equilibrium, this gives a vector polarization of $0.000515 . \quad 80$

5.5 Data points for vector polarization and alignment. Points lying on the smooth curve satisfy the Spin Temperature Theorem. . . . . . . . 82

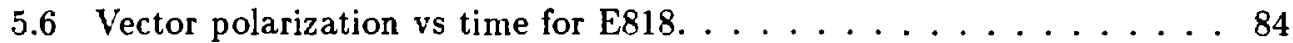

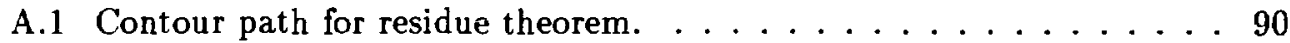

C.1 Range of $s$ and $s \ldots \ldots \ldots \ldots \ldots \ldots \ldots \ldots$

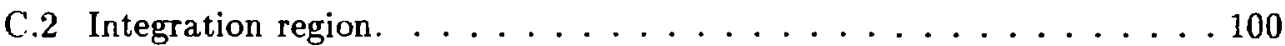

D.1 Schematic representation of $\mathrm{ND}_{3}$ freezing apparatus. . . . . . 111 


\section{Chapter 1}

\section{Introduction}

Nuclear and particle physicists have increasingly found interesting experiments which require the use of polarized targets. This dissertation will be primarily concerned with techniques by which nuclear magnetic resonance (NMR) data may be used to monitor vector and tensor polarizations in solid polarized targets of spin one nuclei. We only consider targets for which the target material consists of large numbers of randomly oriented pieces. We also assume that the nuclei have nonzero quadrupole moments, and that the crystal environments of the nuclei generate electric field gradients which couple to the quadrupole moment. Some crystals (e.g., lithium deuteride) hare symmetry properties which make this coupling zero. We do not consider target materials of this type.

The techniques were developed and tested as a part of a protondeuteron ( $p-d)$ elastic scattering experiment conducted at the Los Alamos 
Meson Physics Facility (LAMPF). This experiment (E818) scattered the 800$\mathrm{MeV}$ polarized proton beam at LAMPF from a solid polarized target consisting of fully deuterated ammonia $\left(\mathrm{ND}_{3}\right)$. The results $[\mathrm{Gu}]$ extend previous measurements of spin correlation coefficients [Ad] to large scattering angles. where multiple scattering, and three body effects are important.

Since the deuteron is the spin one nucleus of interest in E818, we often refer to the spin one nucleus as the deuteron. With the restriction mentioned above, however, our results hold generally for spin one nuclei. Enhanced polarization was produced in the $\mathrm{ND}_{3}$ target through the use of the dynamic nuclear polarization process (DNP). To implement DNP, paramagnetic $\mathrm{F}$ centers must be introduced into the target material. In our case, this was done by irradiating the material in particle beams (see Appendix D). By placing the target in a large magnetic field (25.00 KG for our apparatus) and continuously irradiating the material with microwaves near the electron Larmour frequency $\left(\nu_{L_{e}}\right)$, large polarizations can be achieved. If $\nu_{L_{d}}$ represents the deuteron Larmour frequency, then the largest magnitudes of vector polarization are achieved with microwave frequencies given approximately by $\nu_{L_{e}} \pm \nu_{L_{d}}$. Different choices of sign give different signs of the vector polarization. An adequate theoretical explanation of the DNP is complicated, and will not be further considered here. Discussions can be found in [We], [De] and $[\mathrm{AG}]$.

The magnetic field required for DNP splits the deuteron energy levels into three Zeeman levels. As we will show, this splitting allows us to probe the level populations with NMR techniques. This provides sufficient informa- 
tion to completely characterize the ensemble of deuterons in the target.

In chapter 2 we develop the equations necessary to characterize the polarization state of a spin one polarized target. Chapter 3 describes the ideal NMR signal, and chapter 4 describes a practical electronic circuit which uses these results to determine the target's polarization state. Chapter 5 presents polarization data collected during E818.

Appendix A contains familiar material concerning the KramersKrönig relation, but is included here to establish a result concerning negative frequencies which is needed in the main text. Appendix B establishes our Fourier transform conventions and lists two transforms important to our considerations. Appendix $\mathrm{C}$ contains a straight forward but tedious derivation not readily available in the literature, and appendix $\mathrm{D}$ gives details of our target material preparation. 


\section{Chapter 2}

\section{Spin States for Spin One}

\section{Targets}

The purpose of a polarized target system is to prepare the momentum and spin state of the deuterons which comprise the beam target. In this chapter we describe the formalism necessary to specify the ensemble state of the large number of deuterons present in a macroscopic target.

For describing the spin state, we adopt the view that our target contains an ensemble of deuterons and use the density matrix formalism [To] to specify the ensemble state. In this formalism, scattering experiments are characterized by an $S$ matrix defined by $\rho_{\text {out }}=S \rho_{i n} S^{\dagger}$ where $\rho_{\text {out }}$ and $\rho_{i n}$ are density operators for the final and initial state, respectively. $\rho_{\text {in }}$ can be factored into beam particle and target deuteron parts by $\rho_{\text {in }}=\rho_{\text {beam }} \rho_{\text {deuteron }}$. We are concerned here with preparation and measurement of $\rho_{\text {deuteron }}$. Since 
momentum is negligible for the solid targets which we consider, this amounts to preparing and characterizing the deuteron spin state.

For our purposes we consider the deuteron to be a fundamental particle ${ }^{1}$ of spin one. As such, the spin state of an individual deuteron is characterized by linear combinations of the three eigenstates of $I_{z}$, where $I_{z}$ denotes the $\hat{z}$ component spin operator. These states are denoted

$$
|m\rangle \text { with } m \in\{1,0,-1\} \text {. }
$$

In addition to $I_{z}$ there are companion operators $I_{x}$ and $I_{y}$, which are required to satisfy the commutation relations

$$
\left[I_{x}, I_{y}\right]=i I_{z} \quad(\text { cyclic })
$$

These relations ensure that the triple $\left(I_{x}, I_{y}, I_{z}\right)$ forms a Cartesian vector operator. Since there are three independent eigenstates $(|1\rangle,|0\rangle,|-1\rangle)$ we may consider all operators to operate in three-dimensional Hilbert space.

In the theory of density matricies, each state of the ensemble corresponds to a unique Hermitian matrix called the density matrix for the state. To describe all such matrices in three-dimensional Hilbert space, we need a collection of nine independent Hermitian matrices; then real linear combinations of these matrices will span the set of possible density matrices. This means nine real parameters are needed to specify a general ensemble state.

\footnotetext{
${ }^{1}$ The experimental apparatus rejects inelastic events.
} 
An examination of symmetrized products of $I_{x}, I_{y}$. and $I_{z}$ shows that a basis set of nine matrices can be found which spans the space of all $3 \times 3$ matrices. These matrices are Hermitian (because of the symmetrization) and can be expected to have simple rotational properties (because they are constructed from the $I_{2}^{\prime} s$.)

A convenient collection of matrices for this purpose is the set

$$
1, I_{x}, I_{y}, I_{z}, I_{x y}, I_{x z}, I_{y z}, I_{z z}, I_{o}
$$

where

$$
\begin{aligned}
& I_{i j}=\frac{3}{2}\left(I_{i} I_{j}+I_{j} I_{i}\right)-2 \delta_{i j} 1, \text { for } i, j, \in\{x, y, z\} \\
& \text { and } \\
& I_{0} \equiv I_{x x}-I_{y y} .
\end{aligned}
$$

The $I_{i}$ are Hermitian and thus so are $I_{i j}$ and $I_{o}$.

If the $I_{x}, I_{y}, I_{z}$ are expressed in a basis of $I_{z}$ eigenstates, and the $I_{i}$ are explicitly computed using equation (2.2), the collection (2.1) is seen to provide a complete and independent set of matrices whoses linear combinations with real coefficients span the set of Hermitian $3 \times 3$ matrices. This can be verified by inspection of the explicit representation listed in Fig. 2.1.

Under the real inner product defined by $(a, b)=\operatorname{tr}(a b)$ the collection (2.1) forms an orthogonal set which spans the space of Hermitian operators. We have

$$
\left(I_{\alpha}, I_{\beta}\right) \equiv \operatorname{tr} I_{\alpha} I_{\beta}=c_{(\alpha)\{\beta)} \delta_{\alpha, \beta} \quad \text { (no sum) }
$$




$$
\begin{gathered}
1=\left(\begin{array}{ccc}
1 & 0 & 0 \\
0 & 1 & 0 \\
0 & 0 & 1
\end{array}\right), I_{x}=\frac{1}{\sqrt{2}}\left(\begin{array}{lll}
0 & 1 & 0 \\
1 & 0 & 1 \\
0 & 1 & 0
\end{array}\right) \\
I_{y}=\frac{i}{\sqrt{2}}\left(\begin{array}{ccc}
0 & -1 & 0 \\
1 & 0 & -1 \\
0 & 1 & 0
\end{array}\right), I_{z}=\left(\begin{array}{ccc}
1 & 0 & 0 \\
0 & 0 & 0 \\
0 & 0 & -1
\end{array}\right) \\
I_{x y}=\frac{3 i}{2}\left(\begin{array}{rrr}
0 & -1 \\
0 & 0 & 0 \\
1 & 0 & 0
\end{array}\right), I_{x z}=\frac{3}{2 \sqrt{2}}\left(\begin{array}{ccc}
0 & 1 & 0 \\
1 & 0 & -1 \\
0 & -1 & 0
\end{array}\right) \\
I_{y z}=\frac{3 i}{2 \sqrt{2}}\left(\begin{array}{rrr}
0 & -1 \\
1 & 0 & 1 \\
0 & -1 & 0
\end{array}\right), \quad I_{z z}=\left(\begin{array}{ccc}
1 & 0 & 0 \\
0 & -2 & 0 \\
0 & 0 & 1
\end{array}\right) \\
I_{0}=3\left(\begin{array}{lll}
0 & 0 & 1 \\
0 & 0 & 0 \\
1 & 0 & 0
\end{array}\right)
\end{gathered}
$$

Figure 2.1: Basis matrices for density operator. 
where $\alpha, 3 \in\{1, x, y, z, x y, x z, y z, z z, o\}$ and

$$
\begin{gathered}
c_{x y, x y}=c_{y z, y z}=c_{x z, x z}=\frac{9}{2} \\
c_{x, x}=c_{y, y}=c_{z, z}=2 \\
c_{z z, z z}=6 \\
c_{0,0}=18 \\
c_{1,1}=3 .
\end{gathered}
$$

These properties can be verified by direct computation. We also note that except for 1 all basis matrices are traceless.

The target polaization apparatus consists partly of a strong uniform magnetic field. which defines an axis of cylindrical symmetry. We expect the deuteron ensemble and the corresponding density matrix to also have this symmetry. To find the symmetric form of the density matrix, we first consider a general ciensity matrix, which as for any Hermitian operator may be written

$$
\rho=a_{1} 1+a_{x} I_{x}+a_{y} I_{y}+a_{z} I_{z}+a_{x y} I_{x y}+a_{z z} I_{z z}+a_{y z} I_{y z}+a_{z z} I_{z z}+a_{y} I_{y}
$$

The a coefficients will be restricted by the requirement that $\rho=\rho^{\prime}$ where $\rho^{\prime}$ is a rotated density matrix. For rotations of angle $\theta$ about the $\dot{z}$ axis we must have

$$
\rho^{\prime}=e^{\mid \theta I_{x}} \rho e^{-19 I_{x}}
$$

since $I_{z}$ generates rotations about $\bar{z}$. It is sufficient to consider infinitesimai rotations, which simplifies (2.5) to

$$
\left.\rho^{\prime}=\rho+i \theta_{[}^{\prime} I_{z} \cdot \rho\right]
$$




$$
\begin{aligned}
=\rho+i \theta\{\quad & a_{1}\left[I_{z}, \mathbf{1}\right]+a_{x}\left[I_{z}, I_{x}\right]+a_{y}\left[I_{z}, I_{y}\right]+a_{z}\left[I_{z}, I_{z}\right]+a_{x y}\left[I_{z} \cdot I_{x y}\right] \\
& \left.+a_{x z}\left[I_{z}, I_{x z}\right]+a_{y z}\left[I_{z}, I_{y z}\right]+a_{z z}\left[I_{z}, I_{z z}\right]+a_{o}\left[I_{z}, I_{o}\right]\right\} .
\end{aligned}
$$

Explicit evaluation of the commutators gives

$$
\begin{gathered}
{\left[I_{z}, 1\right]=0,} \\
{\left[I_{z}, I_{x}\right]=i I_{y},} \\
{\left[I_{z}, I_{y}\right]=-i I_{x},} \\
{\left[I_{z}, I_{z}\right]=0,} \\
{\left[I_{z}, I_{x y}\right]=-i I_{o},} \\
{\left[I_{z}, I_{x z}\right]=i I_{y z},} \\
{\left[I_{z}, I_{y z}\right]=-i I_{x z},} \\
{\left[I_{z}, I_{z z}\right]=0,} \\
{\left[I_{z}, I_{o}\right]=4 i I_{x y} .}
\end{gathered}
$$

Using these results we obtain

$$
\rho^{\prime}=\rho+i \theta\left\{i a_{x} I_{y}-i a_{y} I_{x}-i a_{x y} I_{0}+i a_{x z} \dot{I}_{y z}-i a_{y z} I_{x z}+4 i a_{0} I_{x y}\right\}
$$

Since cylindrical symmetry requires $\rho^{\prime}=\rho$ for all $\theta$, the second term must be zero. All the operators occurring in this term are independent, so we may conclude

$$
0=a_{x}=a_{y}=a_{x y}=a_{x z}=a_{y z}=a_{o} .
$$

From (2.4) we see $\rho$ must take the form

$$
\rho=a_{1} \mathbf{1}+a_{z} I_{z}+a_{z z} I_{z z}
$$


The density matrix formalism requires $\operatorname{tr} \rho=1$. Using the orthogonality properties (2.3) we compute

$$
1=\operatorname{tr} \rho=a_{1} \operatorname{tr} 1=3 a_{1} .
$$

So, $a_{1}=\frac{1}{3}$. Similarly, a physical interpretation of the remaining $a$ coefficients is obtained by using the formalism to compute the following expectation values

$$
\begin{gathered}
\left\langle I_{z}\right\rangle=\operatorname{tr}\left(\rho I_{z}\right)=2 a_{z} \\
\left\langle I_{z z}\right\rangle=\operatorname{tr}\left(\rho I_{z z}\right)=6 a_{z z} .
\end{gathered}
$$

Thus for cylindrically symmetric systems, $\rho$ becomes

$$
\rho=\frac{1}{3} 1+\frac{1}{2}\left\langle I_{z}\right\rangle I_{z}+\frac{1}{6}\left\langle I_{z z}\right\rangle I_{z z}
$$

In accordance with convention [B1] we define the polarization, $P$ and the alignment, $A$ by

$$
\begin{gathered}
P \equiv\left\langle I_{z}\right\rangle \\
A \equiv 3\left\langle I_{z}^{2}\right\rangle-2=\left\langle I_{z z}\right\rangle .
\end{gathered}
$$

$P$ and $A$ are also known respectively as the vector and tensor polarizations. The last equality of (2.10) follows from the definition of $I_{z z}$. Because $I_{z}$, and $I_{z}^{2}$, satisfy

$$
\begin{gathered}
-1 \leq\left\langle I_{z}\right\rangle \leq 1 \\
0 \leq\left\langle I_{z}^{2}\right\rangle \leq 1
\end{gathered}
$$

we have

$$
-1 \leq P \leq 1
$$




$$
-2 \leq A \leq 1
$$

Using (2.9) we finally write the most general cylindrically symmetric density matrix as

$$
\rho=\frac{1}{3}\left(1+\frac{3}{2} P I_{z}+\frac{1}{2}-A I_{z z}\right)
$$

or explicitly

$$
\rho=\frac{1}{6}\left(\begin{array}{ccc}
2+A+3 P & 0 & 0 \\
0 & 2-2 A & 0 \\
0 & 0 & 2+A-3 P
\end{array}\right) .
$$

Note that the upper left hand corner refers to the $\{1|\rho| 1\rangle$ matrix element where we denote eigenstates of $I_{z}$ by $|m\rangle$ for $m \in 1,0,-1$. The allowed values of $A$ and $P$ are restricted by the requirement that diagonal elements of a density matrix be positive. This, and (2.14) restrict $A$ and $P$ to lie within the triangular region shown in Fig. 2.2. Pure states of a density matrix are characterized by the requirement that $\rho^{2}=\rho$. By subjecting (2.16) to this constraint, it is straight forward to prove that there are only three cylindrically symmetric pure states: $P=0$ with $A=-2$, and $P= \pm 1$ with $A=1$. These pure states occur at the vertices of the triangle in Fig. 2.2

In principle, an ensemble could be constructed which has $n_{+}$deuterons in the $|1\rangle$ state, $n_{0}$ in the $|0\rangle$ state and $n_{-}$in the $|-1\rangle$ state, and no deuterons in any other state (such as $|1\rangle+|0\rangle$ ). From the theory of density 


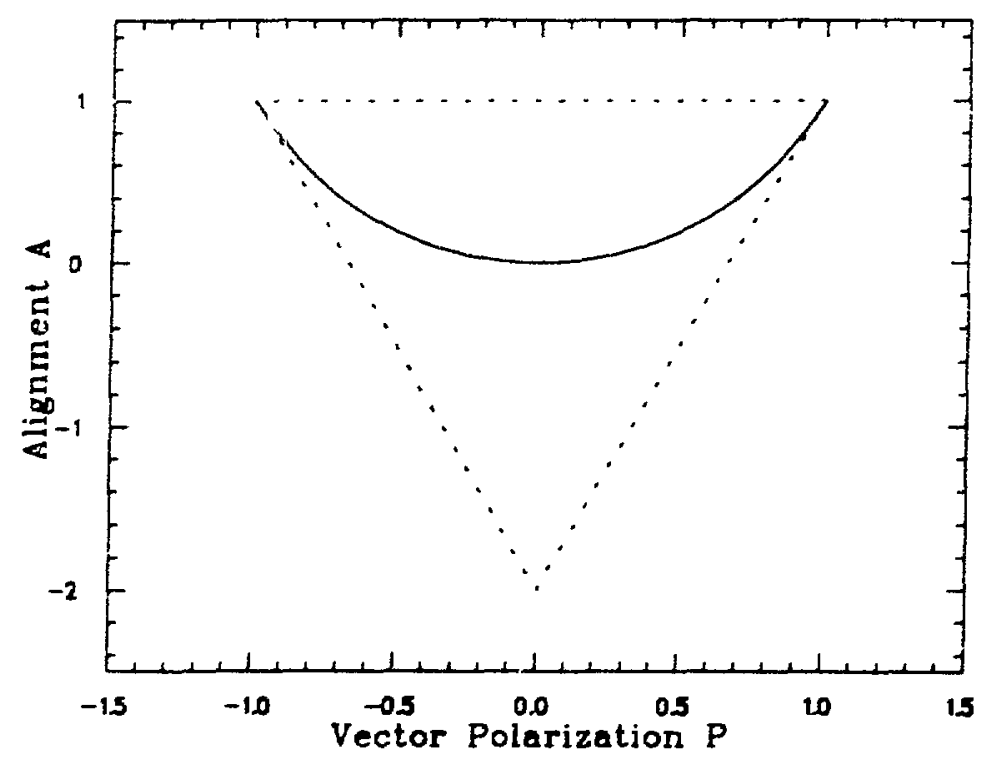

Figure 2.2: Allowed values of alignment, $A$, and vector polarization, $P$. for a rylindrically symmetric system lie on and inside the triangle. Values lying on the curve can be described by an effective temperature which is described in the text. Pure states lie at the vertices. 
matrices, the corresponding $\rho$ is given by

$$
\rho=\frac{1}{N}\left(\begin{array}{ccc}
n_{+} & 0 & 0 \\
0 & n_{0} & 0 \\
0 & 0 & n_{-}
\end{array}\right)
$$

where $N=n_{+}+n_{0}+n_{-}$. Comparing with (2.16) we can solve for $P$ and $A$ to write

$$
\begin{gathered}
P=\frac{n_{+}-n_{-}}{N} \\
A=\frac{n_{+}-2 n_{0}+n_{-}}{N}=3 \frac{n_{+}+n_{-}}{N}-2 .
\end{gathered}
$$

We also list the inverted equations explicitly

$$
\begin{gathered}
\frac{n_{+}}{N}=\frac{1}{3}+\frac{-A}{6}+\frac{P}{2} \\
\frac{n_{-}}{N}=\frac{1}{3}+\frac{A}{6}-\frac{P}{2} \\
\frac{n_{0}}{N}=\frac{1-A}{3} .
\end{gathered}
$$

Restricting $A$ and $P$ to the allowed values indicated in Fig. 2.2 ensures that $n_{+}, n_{-}$, and $n_{0}$ are all positive. Summing the above three equations confirms that $n_{+}+n_{-}+n_{0}=N$.

From this we conclude that any ensemble describable by $n_{+}, n_{0}$, and $n_{-}$can be put in the form of (2.16) and is therefore cylindrically symmetric. The converse is not true. Cylindrically symmetric ensembles exist for instance, which have some deuterons in the state $|1\rangle+|0\rangle$. However, such ensembles still must have a density matrix of the form (2.16), and therefore there must exist a different ensemble which is described by $n_{+}$, and $n_{0}, n_{-}$ 
and has the same density matrix. We know from density natrix theory that all experiments which average over ensembles of states, give identical physical results whenever the corresponding ensembles have identical density matrices. Thus when considering cylindrically symmetric ensembles, we are justified in assuming they have the form given in (2.17).

Now we consider the more restrictive case where $\rho$ takes the special form

$$
\rho=c\left(\begin{array}{ccc}
r & 0 & 0 \\
0 & 1 & 0 \\
0 & 0 & \frac{1}{r}
\end{array}\right)
$$

and where $r$ is some positive number. This will be the case for instance, if the system is in thermodynamic equilibrium [To-2]. and its Hamiltonian commutes with $I_{z}$, and has three equally spaced energy levels. Non-equilibrium systems may also have this density matrix. The constant $c$ is determined by the condition

$$
1=\operatorname{tr} \rho=c\left(r+1+\frac{1}{r}\right)
$$

and therefore

$$
c=\frac{r}{r^{2}+r+1}
$$

From (2.18) and (2.19) we have

$$
\begin{gathered}
P_{\text {Boltz }}=\operatorname{tr}\left(\rho I_{z}\right)=\frac{r^{2}-1}{r^{2}+r+1} \\
A_{\text {Bolt } z}=\operatorname{tr}\left(\rho I_{z z}\right)=\frac{r^{2}-2 r+1}{r^{2}+r+1} .
\end{gathered}
$$

We use the convention that quantities subscripted with Boltz have density matrices of the form given in (2.23). A simple analysis shows that for positive 
$r, P_{\text {Boltz }}$ monotonically increases from -1 to 1 , and $A_{\text {Boltz }}$ takes a minimum value of zero at $r=1$ (see Fig. 2.3). Note in contrast, that the general cylindrically symmetric $\rho$ allows $A$ to be as small as -2 (see 2.14).

From (2.24) and (2.25) we obtain

$$
\frac{A_{\text {Bolt } z}}{P_{\text {Boltz }}}=\frac{r-1}{r+1}
$$

which emphasizes that the assumption of (2.23) forces a dependence between $P_{B o l t z}$ and $A_{\text {Boltz }}$. To find the explicit form of this relationship, compare (2.16) and (2.23) to write

$$
\frac{2+A_{\text {Boltz }}+3 P_{\text {Bolt } z}}{2-2 . A_{\text {Bolt } z}}=\frac{2-2 A_{\text {Boltz }}}{2+A_{\text {Boltz }}-3 P_{\text {Bolt } z}} .
$$

This can be solved for $A_{B o l t z}$ giving

$$
A_{\text {Boltz }}=2-\sqrt{4-3 P_{\text {Bollz }}^{2}}
$$

Also by comparing (2.17) and (2.23) we see

$$
\frac{n_{+}}{n_{0}}=\frac{n_{0}}{n_{-}}=r,
$$

thus $r$ may be interpreted as the ratio of populations.

Now consider an ensemble of deuterons which have been placed in a magnetic field which is uniform and oriented along $\hat{z}$. This configuration is important in the polarization process. The Hamiltonian takes the Zeeman form

$$
\mathcal{H}_{z e e}=-\mu \bar{I} \cdot \bar{B}=-\mu I_{z} B_{z}
$$

where $\mu$ is the magnetic moment of the deuteron and $\bar{B}$ is the magnetic field. We see that the Zeeman energy eigenstates of $\mathcal{H}_{z e e}$ are identical with those of 

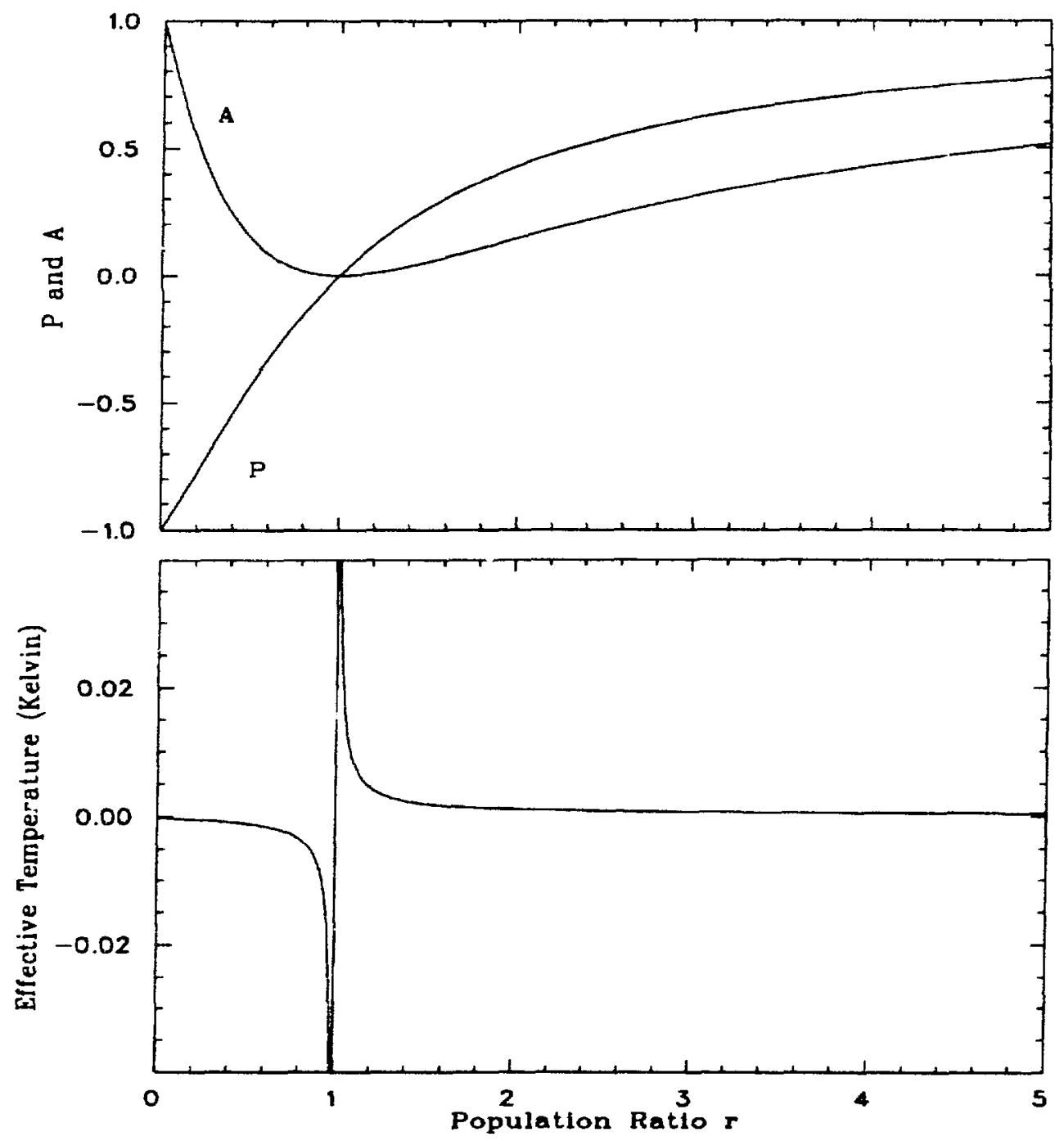

Figure 2.3: Polarization, alignment, effective temperature, $T_{\text {efd }}$, vs. the population ratio, $r$. The $T_{e f f}$ curve assumes $B_{z}=25.00 \mathrm{hG}$ and the deuteron magnetic moment of 0.85742 n.m. $P$ and $A$ are computed with (2.21) and (2.25). 
$I_{z}$. For the case of thermal equilibrium $r$ is given by the Boltzmann factor,

$$
r=e^{\frac{A E}{k T}}=e^{\frac{\frac{H B_{2}}{k T}}{k T}}
$$

where $k$ is the Boltzmann constant and $T$ is the ensemble temperature. Equations (2.24) and (2.25) become

$$
\begin{aligned}
P_{\text {Boltz }} & =\frac{4 \tanh \left(\frac{\mu B_{z}}{2 k T}\right)}{3+\tanh ^{2}\left(\frac{\mu B_{z}}{2 k T}\right)} \\
A_{\text {Boltz }} & =\frac{4 \tanh ^{2}\left(\frac{\mu B_{z}}{2 k T}\right)}{3+\tanh ^{2}\left(\frac{\mu B_{z}}{2 k T}\right)}
\end{aligned}
$$

and we see that for fixed magnetic field the polarization state is completely determined by the temperature.

For systems which are not in thermal equilibrium, but for which (2.23) is still valid, (2.30) can be inverted to express an effective temperature in terms of $P_{\text {Boltz }}$. Using the identity $\tanh ^{-1} x=\frac{1}{2} \ln \frac{1+x}{1-x}$, it is straight forward to show

$$
T_{e f f}=\frac{\mu B_{z}}{k} \frac{1}{\ln \left(\frac{P_{\text {Bolkz }}+2-\sqrt{4-3 P_{\text {Boltz }}^{2}}}{P_{\text {Boltz } z}-2+\sqrt{4-3 P_{\text {Boltz }}^{2}}}\right)} .
$$

This is useful for relating polarizations of different spin species when they are known to have the same $T_{\text {eff. }}$ (See Fig. 2.3). This is the case, for instance, in systems with several nuclear species, and for which the equal spin temperature theorem is applicable [Go].

In summary, we note that cylindrical symmetry has reduce the number of required ensemble parameters from nine to two. These parameters are known as the vector polarization $(P)$ and alignment $(A)$. We will see in the next chapter that nuclear magnetic resonance measurements contain enough 
information to determine both $P$ and $A$. For cases of thermodynamic equilibrium, $P$ and $A$ are given by (2.30) and (2.31) and a single parameter which may be taken to be the temperature, desrribes the ensemble. This is also true for nonequilibrium states whose density matrices take the form of (2.23). Such states may be parameterized by an effective temperature as given by (2.32). 


\section{Chapter 3}

\section{Absorption Line-shape for Spin One}

In this chapter, we discuss the manner in which information about the polarization state of the target may be extracted from characteristics of the deuteron NMR signal. As with the previous chapter, the results are also true for any spin one nucleus. In the next chapter, we will consider the details of the electronic equipment needed to collect the NMR data. A necessary prerequisite to this, is a knowledge of the photon absorption rate as a function of frequency. We will refer to this function as the line-shape. Since an explicit derivation is not readily available ${ }^{1}$ in the literature, this is the subject of this chapter.

\footnotetext{
${ }^{1}$ See however, $[B R]$
} 
We are primarily interested in line-shapes for targets which consist of large numbers of small chunks of randomly oriented material. In the literature [CR-1] such line-shapes are sometimes called powder patterns, and we will use this terminology, even though our chunks are on the order of $1 \mathrm{~mm}$ in diameter. Each chunk is assumed to consist of a number of randomly oriented domains, in which the crystal structure is perfect. We assume that a negligible number of deuterons reside near domain boundaries. We also assume initially that all deuterons are located in rotationally equivalent sites. Conceptually this means that the electric field gradients at each deuteron site could be brought to equivalence by some proper Euclidean rotation. Physically this could not be done without destroying the crystal, since different rotations may be required at different deuteron sites. In general, it will not be true that all deuteron sites are. equivalent in this way. This more general case may be handled by dividing the deuteron sites into rotationally equivalent classes, applying the analysis of this chapter to find the line-shapes for each class, and then superposing the result. A class of rotationally equivalent sites is sometimes referred to as a "bond type." Because of the structure of free $\mathrm{ND}_{3}$ molecules, we expect to have a single bond type in the case of greatest interest here, and indeed we did not find evidence of multiple bond types in $\mathrm{ND}$. 


\subsection{Qualitative Description}

We begin with a qualitative description of the relevant parameters and relate them to simple features of the line-shape. We follow this with a precise mathematical discussion.

For a target consisting of a large number of randomly oriented chunks, the parameters relevant to the line-shape are the $P$ and $A$ described in the previous chapter, two constants $\eta, \nu_{Q}$ which depend on the target material (e.g., deuterated hydrocarbons or $\mathrm{ND}_{3}$ ), and a line broadening parameter, $\sigma$. The applied magnetic field, $B$, is also a parameter, but other things being constant, changes in $B$ have the trivial effect of shifting the absorption curve along the frequency axis without otherwise changing the shape. Such shifts do not interest us here.

The parameter $\sigma$ is a phenomenological constant which describes the average variation in local magnetic field at different points within the target. These field variations result from magnetic fields of electrons and nuclei which surround each point in the target. $\sigma$ depends on target polarization [Ha], but within our range of interest it is found to be relatively small compared to the line-shape width, and constant. The effect of $\sigma$ is to produce a slight rounding of the otherwise sharp shoulders and peaks of the absorption signal. Mathematically, we will incorporate the effects of these field variations by first finding the line-shape for the ideal case of no field variations, then convolving 
the ideal shape with a Gaussian

$$
\delta_{\sigma}(x)=\frac{1}{\sqrt{2 \pi \sigma^{2}}} \exp \left(\frac{-x^{2}}{2 \sigma^{2}}\right) .
$$

The Lorentzian $\left(\delta_{\sigma}(x)=\frac{\sigma}{\pi\left(x^{2}+\sigma^{2}\right)}\right)$ is an alternative form which would make no difference to the results discussed in this chapter.

The parameter $\nu_{Q}$ is proportional to the product of the deuteron's intrinsic electric quadrupole moment, multiplied by the strength of the electric field gradient seen by the deuteron at its rest position in the lattice. $\nu_{Q}$ depends on the symmetries of the electric field gradient at the deuteron site. More precise definitions of $\eta$ and $\nu_{Q}$ will be given later (see respectively, pages 32 and 39 ).

Fig. 3.1 shows a typical powder pattern. We will prove that

$$
\begin{aligned}
P & \propto \text { integral of powder pattern, } \\
A & =\left(\frac{h_{1}-h_{2}}{h_{1}+h_{2}}\right) P \\
\nu_{Q} & =\left|\Delta \nu_{s}\right| \\
\eta & =1-2\left|\frac{\Delta \nu_{p}}{\Delta \nu_{s}}\right|=1-2 \frac{\left|\Delta \nu_{p}\right|}{\nu_{Q}}
\end{aligned}
$$

where the $\Delta \nu$ subscripts indicate peak ( $\mathrm{p}$ ) or shoulder (s). Thus in principle, the powder pattern contains sufficient information to determine all of the parameters needed to describe the target. Though the above formulas are useful for rough approximations, it must be emphasized that in a realistic experimental setting, distortions from the measurement apparatus often make accurate determinations of $\nu_{p}, \nu_{s}, h_{1}, h_{2}$ and especially the proportionality constant for $P$ difficult. The next chapter describes a method appropriate 

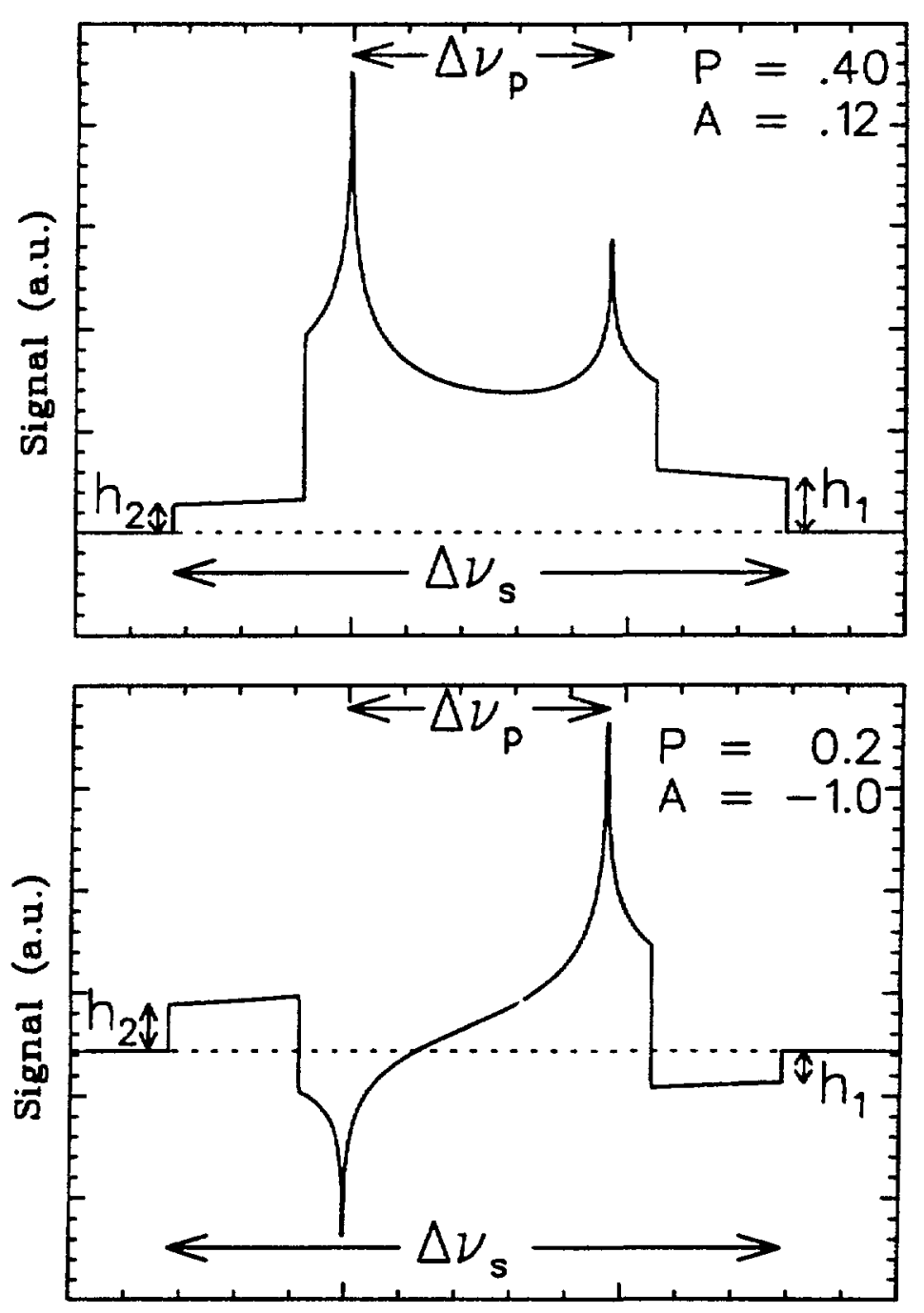

Frequency

Figure 3.1: Typical powder patterns showing definitions of $h_{1}, h_{2}, \Delta \nu_{p}$, and $\Delta \nu_{s}$. Note that $h_{1}$ and $h_{2}$ are negative when they extend below the zero axis. The dotted line indicates zero. 
to our apparatus. This method involves a curve fitting scheme and it is therefore useful to understand the usual line-shape parameters $\nu_{Q}$ and $\eta$ in terms of such simply identifiable properties of the line-shape, as $\Delta \nu_{s}$ and $\Delta \nu_{p}$.

There are two restrictions on the use of this formalism. One is that (3.3) is indeterminate whenever $P=0$. The second is that (3.3), (3.4), and (3.5) are undefined whenever $\nu_{Q}=0$. This happens when the target nucleons are located at sites for which the electric field gradient is zero. (In a notation introduced below, this means $V_{\alpha \beta}=0$ ). For instance, this occurs for crystals with cubic symmetry, such as the lithium halides. In this dissertation, we do not consider further, targets for which $\nu_{Q}=0$.

\subsection{Mathematical Details}

To compute the line-shape (i.e., absorption curve) we must know the energy levels available to each deuteron. For that, we need a Hamiltonian which describes the interaction between the deuteron.and the electromagnetic field at its lattice site. We begin by writing the most general form of orientation dependent interaction which can exist between a charged spin one particle and a static electromagnetic field. The Hamiltonian must take the form

$$
\mathcal{H}=-\bar{\mu} \cdot \bar{B}+\frac{1}{6} \sum_{\alpha \beta} \hat{Q}_{\alpha \beta} V_{\alpha \beta},
$$

where $\bar{\mu}$ is the magnetic moment of the particle, $\bar{B}$ is the magnetic field at the particle, and $V_{\alpha \beta}$ is defined by

$$
V_{\alpha \beta} \equiv \frac{\partial^{2} \phi}{\partial x^{\alpha} \partial x^{\beta}}
$$


where $\phi$ is the electric potential due to charges external to the deuteron. For spin one, the operator $\hat{Q}_{\alpha \beta}$ is defined by

$$
\hat{Q}_{\alpha \beta} \equiv e Q\left\{\frac{3}{2}\left(I_{\alpha} I_{\beta}+I_{\beta} I_{\alpha}\right)-2 \delta_{\alpha \beta} \mathbf{1},\right\}
$$

where $e$ is the electron charge, $Q$ is the quadrupole moment of the deuteron. and the $I_{\alpha}$ are the spin operators defined in the previous chapter. That $\mathcal{H}$ must take this restricted form is a consequence of the well established fact that ground states of nuclei are nondegenerate states of well defined parity. For such states, all electric multipoles vanish unless the multipole order can be written as $2^{l}$, where $l$ is an even non-negative integer which is less than or equal to $2 I$ (twice the particle spin) [Rams]. For spin one, this leaves only electric monopole and electric quadrupole terms. We drop the monopole term since it has no orientation dependence, and we are left with the second term in (3.6).

The magnetic multipoles are similar except $l$ must be odd. For spin one this leaves only magnetic dipole, which appears as the first term in (3.6). The form of $\hat{Q}_{\alpha \beta}$ given in (3.7), where it is expressed explicitly in terms of the spin operators $I_{\alpha}$, results from the Wigner-Eckhart Theorem [Sl-1].

To further simplify (3.6), we assume that the magnetic field $\bar{B}$ is parallel to and close in magnitude to the $25.00 \mathrm{KG}$ magnetic field generated by our polarizing magnet. We also assume that $\bar{B}$ does not depend on how the crystal domains are oriented with respect to the polarizing field. These assumptions are equivalent to assuming that the static magnetic susceptibility tensor is isotropic and close in magnitude to the unit tensor. 
By defining our coordinates so that $\bar{B}$ points in the $\hat{z}$ direction, (3.6) becomes

$$
\begin{aligned}
\mathcal{H} & =-\mu B_{z} I_{z}+\frac{1}{6} \sum_{\alpha \beta} \hat{Q}_{\alpha \beta} V_{\alpha \beta} \\
& \equiv \mathcal{H}_{\text {Zee }}+\mathcal{H}_{\text {quad }}
\end{aligned}
$$

where the terms in the second equation are defined by the corresponding terms in the first. $\mathcal{H}_{\text {Zee }}$ may be called the Zeeman Hamiltonian and $\mathcal{H}_{\text {quad }}$ the quadrupole Hamiltonian. Our measurements confirm that for our $\mathbf{2 5 . 0 0}$ KG polarizing field, $\mathcal{H}_{\text {quad }}$ is small (about $1 \%$ ) compared to $\mathcal{H}_{Z e e}$. We are thus justified in using first order time independent perturbation theory to compute quadrupole corrections to the Zeeman energy levels.

The Zeeman energy levels are given by

$$
E_{Z e e}(m)=\left\langle m\left|\mathcal{H}_{Z e e}\right| m\right\rangle={ }_{\mu} B_{z}\left\langle m\left|I_{z}\right| m\right\rangle
$$

Using the value $\mu_{\text {deuteron }}=0.85742$ nuclear magnetons and $B_{z}=25.00 \mathrm{KG}$ and the explicit form of $I_{z}$ given in the previous chapter, we obtain

$$
\begin{aligned}
E(1) & =\mu_{\text {deut }} B_{z}=0.00342 \mathrm{~cm}^{-1}=6.757 \times 10^{-8} \mathrm{eV} \\
E(0) & =0 \\
E(-1) & =-\mu_{\text {deut }} B_{z}=-0.00342 \mathrm{~cm}^{-1}=-6.757 \times 10^{-8} \mathrm{eV} .
\end{aligned}
$$

The Zeeman energy level diagram is given in Fig. 3.2. The energy difference between the $m=1$ and $m=0$ states is identical to that between the $m=0$ and $m=-1$ states. Therefore the line-shape has a single peak as 


\section{Zeeman}

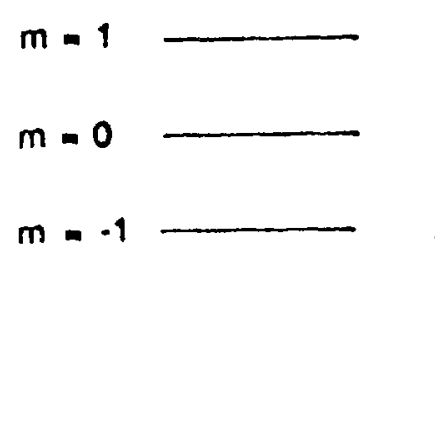

a)
$16.34 \mathrm{MHZ}$

$16.34 \mathrm{MHZ}$

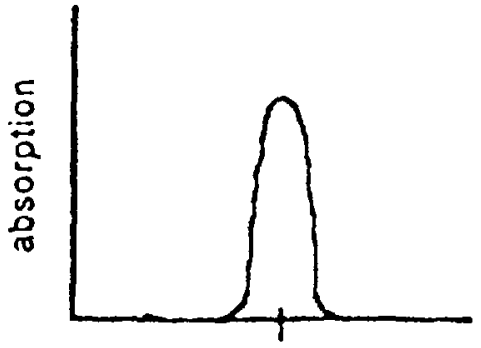

$16.34 \mathrm{MHz}$

b)

Figure 3.2: a) Energy level diagram for $\mathcal{H}_{Z_{e e}}$ for $B_{Z_{e e}}=25.00 \mathrm{KG}$. b) Schematic representation of corresponding line-shape (i.e., a delta function depicted here as a shasp peak).

indicated schematically in Fig. 3.2(b). (We ignore the two photon absorption peak at $32.6 \mathrm{MHz}$ because it is far outside our frequency scan). The quadrupole contribution will split the line-shape into two peaks.

Energy level shifts resulting from $\mathcal{K}_{\text {quad }}$ are given by the perturbation formula

$$
\Delta E(m)=\left\langle m\left|\mathcal{H}_{\text {quad }}\right| m\right\rangle=\frac{V_{a \beta}}{6}\left\langle m\left|\hat{Q}_{\alpha \beta}\right| m\right\rangle
$$

Using well known properties of the $I_{\alpha}$ 's we may explicitly evaluate $\left(m\left|\hat{Q}_{\alpha \beta}\right| m\right)$. 
Table 3.1: Non-zero diagonal elements of $\hat{Q}_{\alpha \beta}$.

$$
\begin{aligned}
& \left\langle 1\left|\hat{Q}_{x x}\right| 1\right\rangle=\left\langle 1\left|\hat{Q}_{y y}\right| 1\right\rangle=-e Q / 2, \quad\left\langle 1\left|\hat{Q}_{z z}\right| 1\right\rangle=c Q \\
& \left\langle 0\left|\hat{Q}_{x I}\right| 0\right\rangle=\left\langle 0\left|\hat{Q}_{y y}\right| 0\right\rangle=\epsilon Q, \quad\left\langle 0\left|\hat{Q}_{z z}\right| 0\right\rangle=-2 \epsilon Q \\
& \left\langle-1\left|\hat{Q}_{x x}\right|-1\right\rangle=\left\langle-1\left|\hat{Q}_{y y}\right|-1\right\rangle=-e Q / 2,\left\langle-1\left|\hat{Q}_{z z}\right|-1\right\rangle=\epsilon Q
\end{aligned}
$$

The non-zero matrix elements are listed in Table 3.1. We see that $\left\langle m\left|\hat{Q}_{\alpha 3}\right| m\right\rangle$ is zero unless $a=3$, and that $\left\langle m\left|\hat{Q}_{x x}\right| m\right\rangle=\left\langle m\left|\hat{Q}_{y y}\right| m\right\rangle$. Thus

$$
\begin{aligned}
\Delta E(m) & =\frac{1}{6}\left\{\left\langle m\left|\hat{Q}_{x x}\right| m\right\rangle V_{x x}+\left\langle m\left|\hat{Q}_{y y}\right| m\right\rangle V_{y y}+\left\langle m\left|\hat{Q}_{z z}\right| m\right\rangle V_{z z}\right\} \\
& =\frac{1}{6}\left\{\left\langle m\left|\hat{Q}_{x x}\right| m\right\rangle\left(V_{x x}+V_{y y}\right)+\left\langle m\left|\hat{Q}_{z z}\right| m\right\rangle V_{z z}\right\}
\end{aligned}
$$

Since there are no external charges at the deuteron site, Poisson's equation gives $\sum_{\alpha} \frac{\partial^{2} \dot{\partial}}{\partial x^{\alpha} \partial x^{\alpha}}=0$, or in our notation

$$
V_{x x}+V_{y y}+V_{z z}=0
$$

Eliminating $V_{x x}+V_{y y}$, we have

$$
\Delta E(m)=\frac{V_{z z}}{6}\left\{\left\langle m\left|\hat{Q}_{z z}\right| m\right\rangle-\left\langle m\left|\hat{Q}_{x x}\right| m\right\rangle\right\} .
$$

In matrix form

$$
\operatorname{diag}\left[\left\langle n\left|\mathcal{H}_{\text {quad }}\right| m\right\rangle\right]=\frac{\epsilon Q V_{z z}}{4}\left(\begin{array}{ccc}
1 & 0 & 0 \\
0 & -2 & 0 \\
0 & 0 & 1
\end{array}\right)
$$

and Fig. 3.3 gives a schematic representation of the energy level shifts, where we have used the definition $\Delta \equiv \frac{e Q V_{2 z}}{4 h}$, where $h$ is Planck's constant. We see 
that the Zeeman peak has been split into two peaks, and that the magnitude of the split is proportional to the two parameters $Q$ and $V_{z z} . Q$ is a fixed property of the deuteron, but $V_{z z}$ depends on how the crystal is oriented with respect to the polarizing $B$ field. Note that the line-shape given in Fig. $3.3(\mathrm{~b})$ is the line-shape which would result from an ensemble of deuterons. all of which have the same $V_{z z}$. In an actual measurement of a perfect single crystal, we would expect several different values of $V_{z z}$ to occur, and each value to give two peaks [Do]. For the powder patterns of interest to us, we eventually average over all orientations, which automatically accounts for the multiple values of $V_{z z}$ which occur in single crystals.

The magnitude of the peaks in the line-shape contain information about the energy level populations. To discuss this, we assume we measure the energy absorbed from a small magnetic field oscillating in the $\hat{x}$ direction (i.e., perpendicular to the polarizing field). For such a field, time dependent perturbation theory shows that the transition rate satisfies [A b-1]

$$
\Gamma_{n \rightarrow m} \propto\left|\left\langle n\left|B_{x} I_{x}\right| m\right\rangle\right|^{2}=\left|B_{x}\right|^{2}\left|\left\langle n\left|I_{x}\right| m\right\rangle\right|^{2} .
$$

From the explicit form of $I_{x}$ given in the previous chapter, we see that the non-zero values of $\left|\left\langle n\left|B_{x} I_{x}\right| m\right\rangle\right|$ are all equal, i.e.,

$$
\left|\left\langle-1\left|B_{x} I_{x}\right| 0\right\rangle\right|=\left|\left\langle 0\left|B_{x} I_{x}\right|-1\right\rangle\right|=\left|\left\langle 0\left|B_{x} I_{x}\right| 1\right\rangle\right|=\left|\left\langle 1\left|B_{x} I_{x}\right| 0\right\rangle\right| .
$$

The rate for a given transition is thus proportional to the population differences. Since for deuterons the lowest Zeeman energy state is $m=1$, we can write for the photon absorption rate $I(\nu)$,

$$
I(\nu) \propto \Gamma\left\{\left(n_{+}-n_{0}\right) \delta_{\sigma}\left(\nu-\nu_{1}\right)+\left(n_{0}-n_{-}\right) \delta_{\sigma}\left(\nu-\nu_{2}\right)\right\}
$$




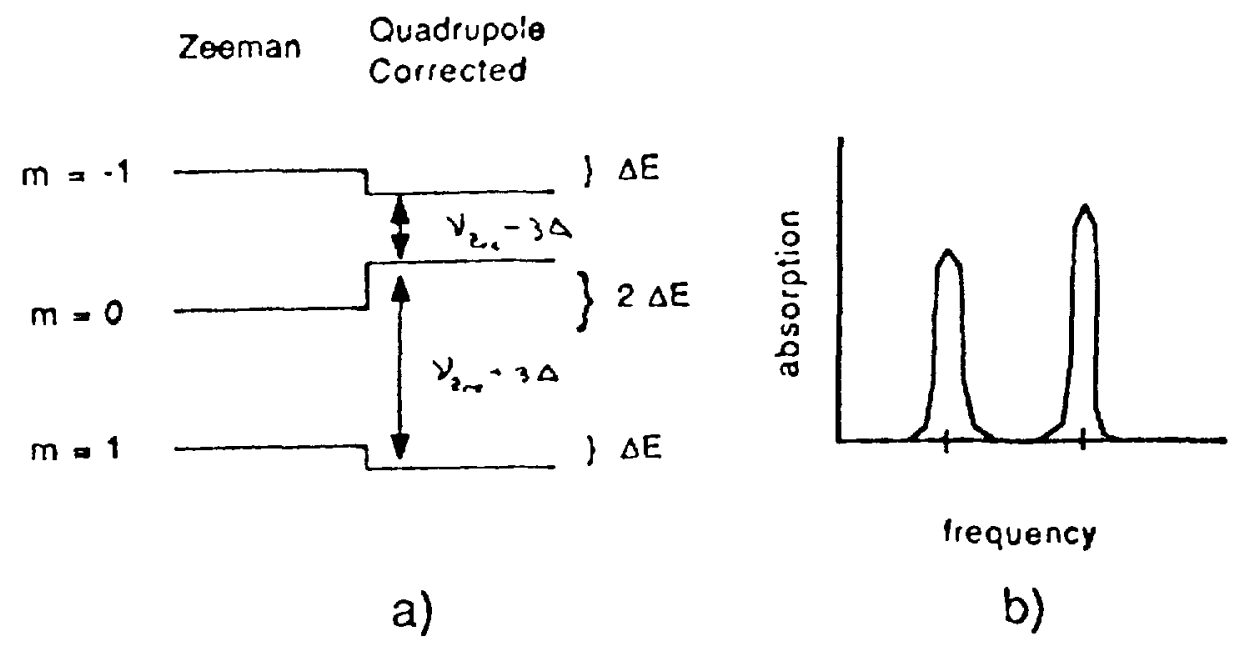

Figure 3.3: a) Level shifts due to $H_{\text {puad }}$. b) Corresponding liw shape. 


$$
=\Gamma\left\{(P-A) \delta_{\sigma}\left(\nu-\nu_{1}\right)+(P+A) \delta_{\sigma}\left(\nu-\nu_{2}\right)\right\},
$$

where $\nu_{Z e e}$ is the Zeeman frequency and $\nu_{1}=\nu_{Z_{e e}}+3 \Delta, \nu_{2}=\nu_{Z \epsilon e}-3 \Delta$, and $P$, and $A$ are defined as in the previous chapter. Absorbing the $\Gamma$ into the proportionality and expanding $\nu_{1}, \nu_{2}$, we have

$$
I(\nu) \times(P-A) \delta_{\sigma}\left(\nu-\nu_{Z e \epsilon}-\frac{3 e Q V_{z z}}{4 h}\right)+(P+A) \delta_{\sigma}\left(\nu-\nu_{Z e \epsilon}+\frac{3 \epsilon Q V_{z z}^{r}}{4 h}\right) .
$$

Thus we have confirmed that for an ensemble of deuterons all with the same value of $V_{z z}$, the line-shape has the form indicated in Fig. 3.3(b), and have demonstrated how the peak heights are related to the polarization state of the ensemble. For later use, we note that since the photon energy is $h \nu$, we have for the absorbed power,

absorbed power $\propto h \nu I$.

In order to average over all orientations in the powder, we must compute the probability distribution for $V_{z z}$. To this end we now show that $V_{z z}$ is essentially described by only two material dependent parameters. Recall $V_{i j} \equiv \frac{\partial^{2} \phi}{\partial x_{i} \partial x_{j}}$. Since $V_{i j}$ is clearly symmetric, there must exist an orthogonal transformation which diagonalizes $V_{i j}$ and satisfies

$$
\left|V_{z^{\prime} z^{\prime}}\right| \geq\left|V_{y^{\prime} y^{\prime}}\right| \geq\left|V_{x^{\prime} x^{\prime}}\right| .
$$

In these coordinates, Poisson's equation becomes

$$
V_{x^{\prime} x^{\prime}}+V_{y^{\prime} y^{\prime}}+V_{z^{\prime} z^{\prime}}=0 .
$$


This constraint leaves only two independent parameters which are by convention taken to be

$$
\begin{aligned}
c q & \equiv V_{z^{\prime} z^{\prime}} \\
\eta & \equiv\left(V_{x^{\prime} x^{\prime}}-V_{y^{\prime} y^{\prime}}\right) / V_{z^{\prime} z^{\prime}}
\end{aligned}
$$

Using (3.11) and (3.12) we solve to obtain

$$
\begin{aligned}
& V_{x^{\prime} x^{\prime}}=\frac{e q(\eta-1)}{2} \\
& V_{y^{\prime} y^{\prime}}=\frac{-\epsilon q(\eta-1)}{2} \\
& V_{z^{\prime} z^{\prime}}=e q .
\end{aligned}
$$

The parameter $\eta$ is known as the "asymmetry parameter" [CR-2]. From (3.10) and (3.11) the reader can show by simple arguments that $\eta$ must satisfy

$$
0 \leq \eta \leq 1, \quad\left(\text { whenever } V_{\mathrm{ij}} \neq 0\right)
$$

The rotation matrix which connects the two coordinate systems may be pararneterized with the three Euler angles $\theta, \phi, \psi$ [Gs]. The $V_{i}$ transform with the tensor rule, so for the $z z$ component we have

$$
V_{z z}=\left(R^{\top}(\theta, \phi, \psi) V^{\prime \prime} R(\theta, \phi, \psi)\right)_{z z}
$$

where we have used matrix multiplication inside the parenthesis, and $R(\theta, 0, \psi)$ and $R^{\top}(\theta, \phi, \psi)$ denote the rotation matrix ${ }^{2}$ and its transpose. Because we 
CIIAPTER 3. ABSORPTION LINE-SHAPE FOR SPIN ONE

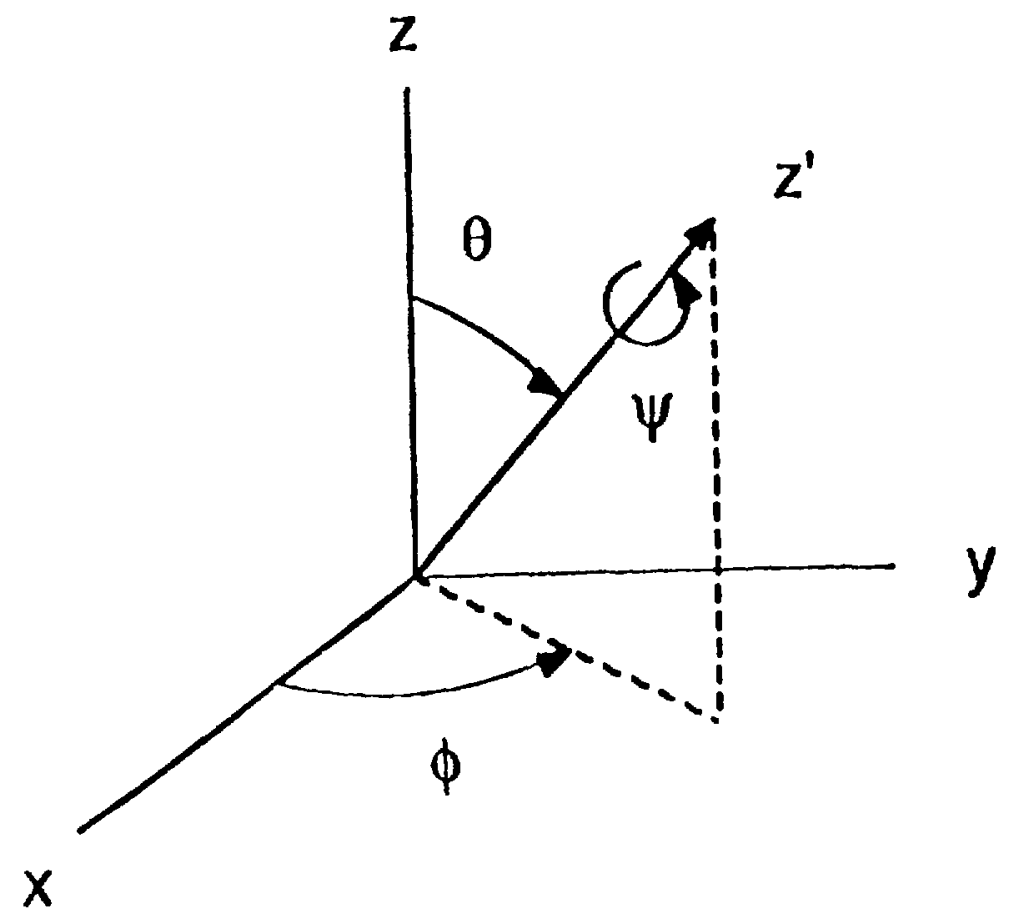

Figure 3.4: Euler angles. 
are only interested in the $z z$ component, the matrix multiplications are fairly simple and give the result

$$
\begin{aligned}
V_{z z} & =\frac{e q}{2}\left(3 \cos ^{2} \theta-1-\eta \sin ^{2} \theta \cos \left(2 \psi^{\prime}\right)\right) \\
& \equiv e q \lambda\left(\theta, \iota^{\circ}\right) .
\end{aligned}
$$

where $\lambda(\theta, \psi)$ is defined by the second equality. Thus $V_{z z}$ is expressed in terms of the material parameters $q$ and $\eta$, and the orientation parameters $\theta$ and $x$.

Next we find the probability densities for $\theta$ and $\leftrightarrow$. For random bead orientations, we expert $\theta$ and $\psi$ to be independent random variables. The physical meaning of $\theta$ is seen in Fig. 3.4 to be the angle between the $z$ and $z^{\prime}$ axis. Random orientation means that the intersection of all the $z^{\prime}$ axes with the unit sphere will uniformly cover that sphere. Thus the probability density for $\theta, \rho_{\theta}$, must satisfy

$$
\rho_{\theta} d \theta \times \text { area associated with } \theta=\sin \theta d \theta .
$$

From the physical meaning of $\psi$. we expect all values of $v$ to be equally likely. therefore

$$
\rho_{\psi} d \psi \times d \psi
$$

Since $\theta$ and $v$ are statistically independent, we get for the joint probabilit: density

$$
\rho(\theta, v) d \theta d v \times \sin \theta d \theta d v .
$$

Yormalizing to one, we obtain the joint probability density for randomly oriented beads

$$
\rho(\theta, v)=\frac{1}{4 \pi} \sin \theta
$$


We must now use this to compute the probability density for $V_{z z}$, which will allow us to average our line-shape over all bead orientations.

We will denote the probability density for $\lambda$ by $f(z)$ and the associated distribution function by $F(z)$. Thus

$$
f(z)=F^{\prime}(z)
$$

where the prime denotes differentiation. The meaning of the distribution function is that $F(x)$ equals the fraction of beads whose $\lambda$ value is smaller than $x$. In Fig. 3.5 we have plotted $\lambda\left(\theta, \psi^{\prime}\right)$ and also several projections of $\lambda$ level-surfaces onto the $\theta-\dot{\psi}$ plane.

To compute $F(z)$ it is clear that we must integrate $\rho(\theta, \psi)$ over the interior of the projected level-surface corresponding to the value $\lambda$. To get $f(z)$ we then simply differentiate. The details of this procedure are straight forward but somewhat tedious and are therefore relegated to appendix C. The result is

$$
f_{\eta}(z)=\left\{\begin{array}{lr}
\frac{1}{\pi \sqrt{\eta(1-z)}} K\left(\frac{(3-\eta)(\eta+1+2 z)}{4 \eta(1-z)}\right) & \text { for }-\frac{\eta}{2}-\frac{1}{2} \leq z \leq \frac{\eta}{2}-\frac{1}{2} \\
\frac{2}{\pi \sqrt{(3-\eta)(\eta+1+2 z)}} K\left(\frac{4 \eta(1-z)}{(3-\eta)(\eta+1+2 z)}\right) & \text { for } \frac{\eta}{2}-\frac{1}{2}<z \leq 1 \\
0 & \text { otherwise }
\end{array}\right.
$$

where

$$
K^{\prime}(x) \equiv \int_{0}^{\pi / 2} \frac{1}{\sqrt{1-x \sin ^{2} \theta}} d \theta
$$

$K^{-}$is a complete elliptic integral of the first kind as defined by Abramowitz 


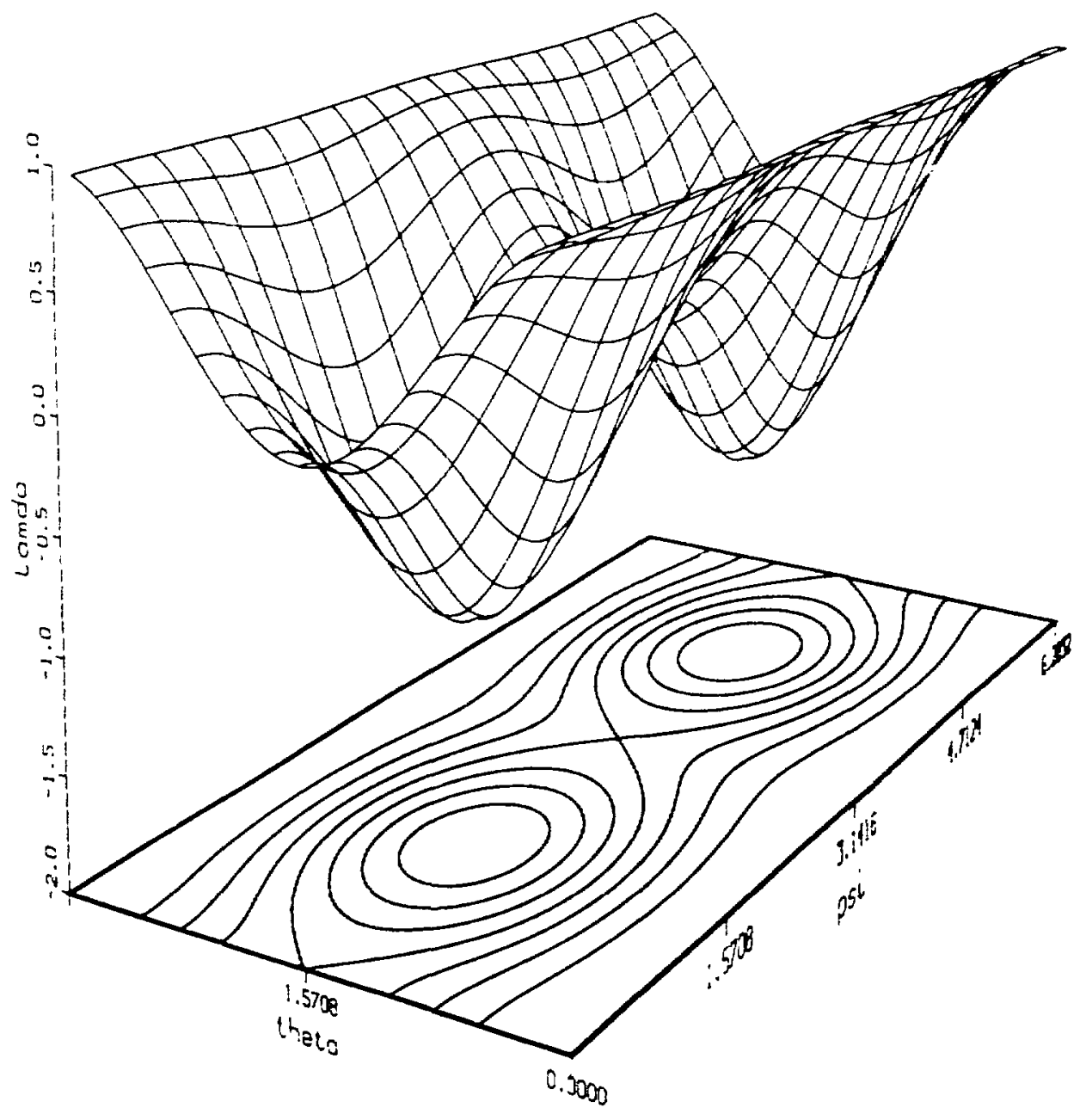

Figure 3.5: $\lambda(\theta, v)$ for $\eta=1$. 
and Stegun [AS]. ${ }^{3}$ We have added the subscript $\eta$ to $f$ to emphasize the dependence on the asymmetry parameter. In Fig. 3.6 we plot $f_{n}$ for sereral values of $\eta$. We note that the quantity $f_{\eta}(z) d z$ is interpreted as the fraction of deuterons which have a $\lambda$ value between $z$ and $z+d z$. From properties of the complete elliptic integral, it is seen that $f_{\eta}$ goes to plus infinity at $z=\frac{\eta-1}{2}$. although it does so in an integrable way, and it may be checked analytically that

$$
\int_{-\infty}^{\infty} f_{\eta}(z) d z=1 . \quad \text { (See appendix C) }
$$

Certain values of these functions are of special interest. $K$ takes the value $\frac{\pi}{2}$ at zero, $+\infty$ at one, and is continuous in between. Thus

$$
\begin{aligned}
f_{\eta}\left(-\frac{\eta}{2}-\frac{1}{2}\right) & =\frac{1}{\sqrt{2 \eta(3+\eta)}} \\
f_{\eta}\left(\frac{\eta}{2}-\frac{1}{2}\right) & =+\infty \\
f_{\eta}(1) & =\frac{2}{\pi \sqrt{(3-\eta)(2+\eta)}} .
\end{aligned}
$$

The density of $V_{z z}$ can now be computed using the density transformation rule

$$
\rho_{V_{x x}}(x)=\frac{1}{e q} f_{\eta}\left(\frac{x}{e q}\right)
$$

This follows since

$$
V_{z z}=e q \lambda
$$

\footnotetext{
${ }^{3}$ Note that $[\mathrm{JE}]$ has a slightly different definition.
} 


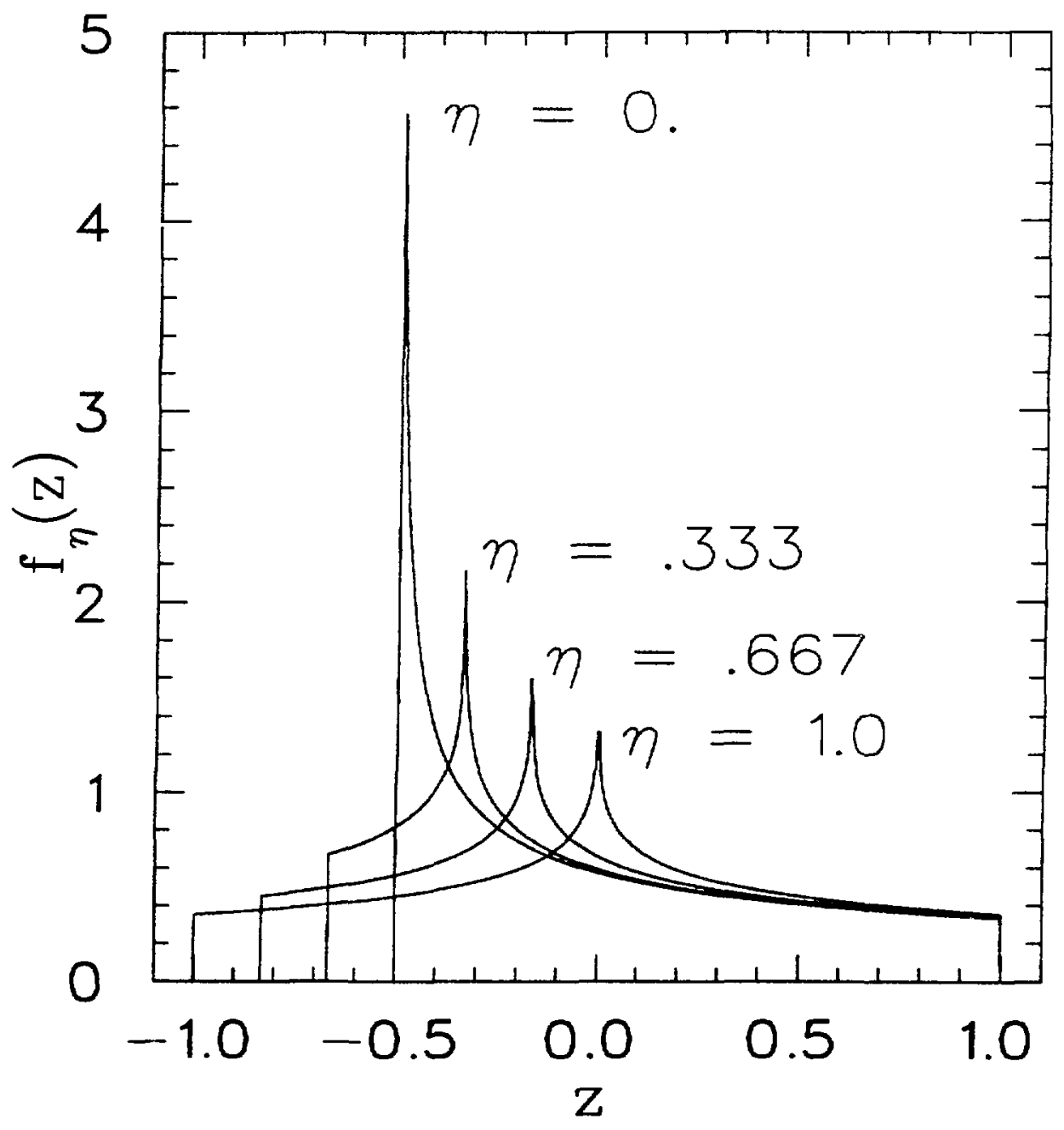

Figure 3.6: Plots of $f_{\eta}(z)$. 
and $f_{11}$ is the density for $\lambda$.

To compute the powder pattern, we have to weight (3.9) with $\rho_{V_{z z}}$ and integrate. Thus we obtain

$$
\begin{aligned}
I_{Q, \eta, \sigma}= & C \int_{-\infty}^{\infty} d z \rho_{V_{z z}}(z)\left\{(P-A) \delta_{\sigma}\left(\nu-\nu^{\prime} Z e e-\frac{3 e Q z}{4 h}\right)\right. \\
& \left.\quad+(P+A) \delta_{\sigma}\left(\nu-\nu_{Z e e}+\frac{3 e Q z}{4 h}\right)\right\} \\
= & C \int_{-\infty}^{\infty} d z f_{\eta}\left(\frac{z}{e q}\right)\left\{(P-A) \delta_{\sigma}\left(\nu-\nu_{Z e e}-\frac{3 e Q z}{4 h}\right)\right. \\
& \left.\quad+(P+A) \delta_{\sigma}\left(\nu-\nu_{Z e \epsilon}+\frac{3 e Q z}{4 h}\right)\right\} \\
=C & \int_{-\infty}^{\infty} d u f_{\eta}(u)\left\{(P-A) \delta_{\sigma}\left(\nu-\nu_{Z e e}-\frac{3 e^{2} Q u q}{4 h}\right)\right. \\
& \left.\quad+(P+A) \delta_{\sigma}\left(\nu-\nu_{Z e e}+\frac{3 \epsilon^{2} Q u q}{4 h}\right)\right\} \\
= & C:\left\{(P-A) g_{\nu_{Q}, \eta, \sigma}^{+}\left(\nu-\nu_{Z e e}\right)+(P+A) g_{\nu_{Q}, \eta, \sigma}^{-}\left(\nu-\nu_{Z e e}\right)\right\}(3.1
\end{aligned}
$$

where

$$
\begin{aligned}
g^{ \pm}(x) \equiv g_{\nu_{Q}, \eta, \sigma}^{ \pm}(x) & \equiv \int_{-\infty}^{\infty} d z f_{\eta}(z) \delta_{\sigma}\left(x \mp \frac{3 e^{2} Q q}{4 h} z\right) \\
& =\frac{2}{\nu_{Q}} \int_{-\infty}^{\infty} d u f_{\eta}\left(\frac{2 u}{\nu_{Q}}\right) \delta_{\sigma}(x \mp u)
\end{aligned}
$$

and $C$ is a constant which fixes the proportionality of (3.9), and we at last define

$$
\nu_{Q} \equiv \frac{3 e^{2} Q q}{2 h}
$$

This definition is common in the literature [Ha] [CR] but not universal [De]. Since the parameter $Q$ only occurs as $\nu_{Q}$, we will denote $I_{Q, \eta, \sigma}$ as $I_{\nu_{Q}, \eta, \sigma}$. We will sometimes drop the subscripts on $g_{\nu_{Q}, \eta, \sigma}$ when it improves readability. Sote that $g_{\nu_{Q}, \eta, \sigma}^{ \pm}$is essentially a smoothed version of $f_{\eta}$. Also note that as 
with $I(v)$, the power absorbed by a powder satisfies

$$
\text { absorbed power } x \text { hw } I_{2,+7, n} \text {. }
$$

It is useful to know the integrals of $g^{ \pm}$.

$$
\begin{aligned}
& \int_{-\infty}^{\infty} d x g_{\nu_{2}, \eta, \sigma}^{z}(x)=\frac{2}{\nu_{Q}} \int_{-\infty}^{\infty} d z f_{n}\left(\frac{2 z}{\nu_{Q}}\right) \int_{-\infty}^{\infty} d x n_{0}(x \mp z) \\
& =\frac{2}{v_{Q}} \int_{-\infty}^{\infty} d z f_{n}\left(\frac{2 z}{v_{0}}\right) \\
& =1 \text {. }
\end{aligned}
$$

Also notice:

$$
\begin{aligned}
& y_{\nu_{z}, \eta, \sigma}^{-}(x)=\frac{2}{\nu_{Q}} \int_{-\infty}^{\infty} d z f_{n}\left(\frac{2 z}{\nu_{Q}}\right) d_{n}(x+z) \\
& =\frac{2}{\nu_{Q}} \int_{-\infty}^{\infty} d z f_{n}\left(\frac{2 z}{\nu_{Q}}\right) \lambda_{1}(-x-z) \text { (by symmetry of } r_{n} \text { ) } \\
& =g_{u_{g} \cdot \pi \cdot g^{+}}^{+}(-x) \text {. }
\end{aligned}
$$

Thus if we know $g^{+}$it is very simple to compute $g^{-}$.

The function $g^{+}$is equivalent to the shape function regrorted by Hamada [Ha] and used by Sperizen [Sp]. To see the equivalence it is necessary tos make some variable substitutions in the integral defining $g^{+}$, which we do in appendix $\mathrm{C}$. This results in definite integrals with finite limits. Wo prefer the form given above because it has the form of a convolution integral. and numerical computation of such integrals is made efficient witis fast Fouries transform techniques. We will discuss this more fully in the next chapter.

We now integrate $I_{\nu a, r .0}$.

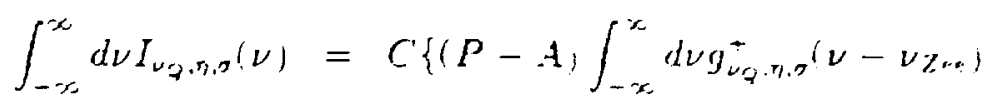




$$
\begin{aligned}
& \left.+(P+A) \int_{-\infty}^{\infty} d \nu_{g_{\nu Q}, \eta \sigma}^{-}\left(\nu-\nu \nu_{e E}\right)\right\} \\
= & C\{(P-A)+(P+A)\} \\
= & 2 C P .
\end{aligned}
$$

This proves (3.2) and suggests the following experimental method for measuring $P$. We simply put the beads in a known state of polarization, usually by waiting for thermal equilibrium at a known magnetic field and temperature, and measure $I_{\nu_{Q}, \eta, \sigma}(\nu)$. Numerical integration of $I_{\nu_{Q}, \eta, \sigma}$ fixes the constant $2 C$ and thereby calibrates further measurements.

To prove equations (3.3), (3.4), and (3.5) we assume the spreading caused by $\delta_{\sigma}$ is negligible, and we ignore it. In this approximation, $\delta_{\sigma}$ becomes a true $\delta$-function and we get from $(3.20)$,

$$
\begin{array}{ll}
g^{+}(x)=\frac{2}{\nu_{Q}} f_{\eta}\left(\frac{2 x}{\nu_{Q}}\right), \quad(\sigma=0) \\
g^{-}(x)=\frac{2}{\nu_{Q}} f_{\eta}\left(-\frac{2 x}{\nu_{Q}}\right), \quad(\sigma=0) .
\end{array}
$$

From (3.16) and (3.17) we see that the maximum and minimum $x$ such that $g^{+}$is non-zero are given by

$$
\begin{aligned}
& x_{\max }^{+}=\frac{\nu_{Q}}{2} \\
& x_{\min }^{+}=-\frac{\nu_{Q}}{2}\left(\frac{\eta+1}{2}\right)
\end{aligned}
$$

and similarly, since $g^{-}(x)=g^{+}(-x)$

$$
\begin{aligned}
& x_{\max }^{-}=\frac{\nu_{Q}}{2}\left(\frac{\eta+1}{2}\right) \leq x_{\max }^{+} \\
& x_{\min }^{-}=-\frac{\nu_{Q}}{2} \leq x_{\min }^{+},
\end{aligned}
$$


where the inequalities follow since $\eta \leq 1$. Since $I_{\nu(, \eta, \tau}$ is a linear combination of $y^{+}$and $g^{-}$, we see that the shoulders of $I_{\nu_{Q}, n, \sigma}$ must occur at

$$
\begin{aligned}
& \nu_{\text {max }}=\nu_{\text {Zee }}+\frac{\nu_{Q}}{2} \\
& \nu_{\text {min }}=\nu_{\text {Zee }}-\frac{\nu_{Q}}{2} .
\end{aligned}
$$

We immediately obtain (3.4) from $\nu_{\max }-\nu_{\min }=\nu_{Q}$. The only difficulty when $\sigma$ is finite. is the determination of the location of the shoulders, which have become slightly rounded.

Equation (3.25) shows that as long as $\eta<1, f_{n}$ has a longer tail to the right of zero, than to the left. This will also be true of $g^{+}$, and the opposite will be true of $g^{-}$, since as we have seen $g^{-}(x)=g^{+}(-x)$. From 3.18 we know that $I_{\nu_{Q}, \eta, \sigma}$ is a linear superposition of $g^{+}$and $g^{-}$, and therefore we conclude that on the extreme right of the (non-zero) powder pattern the signal is proportional to $g^{+}$(because $g^{-}$is zero) and on the extreme left, proportional to $g^{-}$(because $g^{+}$is zero). From (3.18) we see that the $g^{+}$ factor is $P-A$, and the $g^{-}$factor is $P+A$. From Fig. 3.1 we get

$$
\frac{h_{2}}{h_{1}}=\frac{(P-A) g^{-}(\text {extreme left })}{(P+A) g^{+}(\text {extreme right })}=\frac{P-A}{P+A},
$$

since $g^{+}$(extreme right $)=g^{-}($extreme left $)$. Algebraic rearrangement gives

$$
A=\frac{h_{1}-h_{2}}{h_{1}+h_{2}} P
$$

which is just (3.3). As before, finite $\sigma$ adds uncertainty by rounding the shoulders. Notice that this formula is indeterminate when $P=0$, since in this case (3.27) implies $h_{1}+h_{2}=0$. 
For (3.5), we notice that when $\sigma=0$, the peaks in the powder pattern are infinitely high. Thus the location of the peaks in $I_{\nu, \eta, \sigma, \sigma}$ orcur at the same frequency as the peaks in $g^{+}\left(\nu-\nu_{Z_{e e}}\right)$ and $g^{-}\left(\nu-\nu_{Z e e}\right)$. That is

$$
\frac{2}{\nu_{Q}}\left(\nu_{p e a k}^{+}-\nu^{\prime} Z_{\epsilon e}\right)=\frac{\eta-1}{2}
$$

and

$$
\frac{2}{\nu_{Q}}\left(\nu_{p e a k}^{-}-\nu_{Z e e}\right)=-\frac{\eta-1}{2} .
$$

Thus $\eta=1-\frac{2|\Delta u|}{\nu_{Q}}$, where $\Delta \nu=\nu_{\text {prak }}^{-}-\nu_{p e a k}^{+}$, and we have established (3.5). We emphasize that for finite $\sigma$, the location of the $g^{+}$peak will be slightly shifted by the $g^{-}$term and visa verse. Equation $(3.5)$ is then only approximately true.

In summary of this section, we collect the results describing the absorption rate $((3.18),(3.20)$, and $(3.16))$.

$$
I_{\nu_{Q}, \eta, \sigma}(\nu)=C\left\{(P-A) g_{\nu_{Q}, \eta, \sigma}\left(\nu-\nu_{Z \epsilon \epsilon}\right)+(P+A) g_{\nu_{Q}, \eta, \sigma}\left(\nu^{\prime} Z_{e e}-\nu\right)\right\}
$$

where

$$
f_{\eta}(z)=\left\{\begin{array}{lr}
\frac{1}{\pi \sqrt{\eta(1-z)}} K\left(\frac{(3-\eta)(\eta+1+2 z)}{4 \eta(1-z)}\right) & \text { for }-\frac{\eta}{2}-\frac{1}{2} \leq z \leq \frac{\eta}{2}-\frac{1}{2} \\
\frac{2}{\pi \sqrt{(3-\eta)(\eta+1+2 z)}} K\left(\frac{4 \eta(1-z)}{(3-\eta)(\eta+1+2 z)}\right) & \text { for } \frac{\eta}{2}-\frac{1}{2}<z \leq 1 \\
0 & \text { otherwise }
\end{array}\right.
$$

where

$$
K(x) \equiv \int_{0}^{\tau / 2} \frac{1}{\sqrt{1-x \sin ^{2} \theta}} d \theta
$$


and

$$
\eta=1-\frac{2\left|\Delta \nu_{p}\right|}{\nu_{Q}}
$$

$P$ and A are the vector polarization and alignment. The parameter ${ }^{\prime} Q$ is the frequency difference between the powder pattern shoulders, and $\Delta \nu_{p}$ is the frequency difference between peaks. $\nu_{O}$ and $\Delta \nu_{\nu}$ are material parameters which depend only on the chemical environment of the deuterons. The parameter $C$ is a trivial normalization constant.

Finally we mention the possibility of rotationally inequivalent deuteron sites (i.e., different bond types). For this case, $\nu_{Q}$ and $\eta$ (i.e., $\Delta \nu_{p}$ ) clearly depend on the bond type. Hopefully the polarization process is such that $P$ and $A$ do not. The powder pattern is then simply a superposition of the $I_{u_{Q}, \eta, \sigma}$ for each bond type. 


\section{Chapter 4}

\section{Polarization Measurement for}

\section{Spin One}

In the previous chapter we saw that the line-shape for energy absorption from a small oscillating magnetic field applied perpendicular to the polarizing field, contains sufficient information to determine the complete polarization state of the deuteron ensemble. In this chapter, we discuss a practical means of measuring this line-shape and determining polarization.

The oscillating field is developed by means of a radio frequency ( $\mathrm{rf}$ ) coil wound in or near the target material and oriented in such a way as to produce a perpendicular field. Radio frequency energy is supplied to the coil by means of an $\mathrm{rf}$ generator and associated circuitry. The response of this circuit as a function of applied frequency is recorded, and used to determine the absorption line-shape. 
From alternating current (ac) circuit theory, we know that the inductance of the rf coil depends on the susceptibility of the naterial insirle the coil. This may be written $[\mathrm{Ab}][\mathrm{Sl}]$

$$
L=L_{0}(1+4 \pi \gamma(\omega))
$$

where $L$ is the effective inductance, $L_{0}$ is the empty spare inductance, and $\ell$ is the complex susceptibility which depends on the angular frequency $w$. Some authors include a filling factor in (4.1), but we will not need it and include it in the definition of $\chi$. Note that $L$ is in general complex, which means that impedances associated with $L$ contain dissipative components. By convention $\chi$ is written in terms of real and imaginary components as

$$
\chi(\omega)=\chi^{\prime}(\omega)-i \chi^{\prime \prime}(w)
$$

In appendix $A$ we show that $\chi^{\prime}$ and $\chi^{\prime \prime}$ satisfy the integral relation

$$
x^{\prime}(\omega)=\frac{-1}{\pi} \mathcal{P} \int_{-\infty}^{\infty} \frac{\chi^{\prime \prime}(x)}{\omega-x} d x
$$

where $\mathcal{P}$ indicates the principle part of the integral. Such an integral transform is known as a Hilbert transform. To compute (4.3) we must know $\chi^{\prime \prime}$ for negative $\omega$, but we only have experimental access to positive $\omega$. In appendix A we show $\chi^{\prime \prime}(-\omega)=-\chi^{\prime \prime}(\omega)$. Integration of (4.3) gives the result

$$
\int \gamma^{\prime}\left(\omega^{\prime}\right) d \omega=0
$$

That $\chi^{\prime}$ and $\chi^{\prime \prime}$ satisfy (4.3) follows from the physically reasonable assumptions that the current flowing in the electrical cirruit formed by the rf coil and its contents responds to a sinusoidal driving voltage in a linear, causal. 
and time translation-invariant way. With these assumptions the Framer Frönig relation may be applied to prove (4.3). ${ }^{1}$

We now ask how much power is dissipated by a current flowing in the If coil. From ac circuit theory, we have

$$
\begin{aligned}
\text { Power } & =\frac{1}{2} \operatorname{Re}\left(I^{*} V^{\prime}\right) \\
& =\frac{1}{2} \operatorname{Re}\left(I^{*} Z I\right) \\
& =\frac{1}{2} \operatorname{Re}\left(I^{*} i \omega L I\right) \\
& =\frac{1}{2}|I|^{2} \operatorname{Re}(i \omega L) \\
& =\frac{1}{2}|I|^{2} \operatorname{Re}\left(i \omega L_{0}\left(1+4 \pi\left[\chi^{\prime}(\omega)-i \chi^{\prime \prime}(\omega)\right]\right)\right) \\
& =2 \pi|I|^{2} L_{0} \omega \chi^{\prime \prime}(\omega),
\end{aligned}
$$

where $I, V$, and $Z$ are respectively, the complex current, voltage and impedance. Power is seen to be proportional to $\omega \chi^{\prime \prime}(\omega)$. In the frequency range of interest here, the only significant energy absorption mechanisms available in the target material are the nuclear spin transitions described in the previous chapter where we saw Power $\propto \nu I_{\nu_{Q}, \eta, \sigma}$. We must then have

$$
\chi^{\prime \prime}(\omega)=\alpha I_{\nu_{Q}, \eta, \sigma}(2 \pi \omega)
$$

where $\alpha$ is a proportionality constant. The quantity $\alpha$ depends on the density of the deuterons near the rf coil, and is not a priori calculable because of the irregular shape of the target material pieces. It is convenient to use (3.28) to

\footnotetext{
'See appendix A
} 
expand (4.5). and to make the following rearrangements.

$$
\begin{aligned}
& \chi^{\prime \prime}(\omega)=\alpha C\left\{(P-A) g_{\nu_{Q}, \eta, \sigma}\left(\nu-\nu_{Z \epsilon \epsilon}\right)+(P+A) g_{\nu_{Q}, n, \sigma}\left(\nu_{Z \mu,}-\nu\right)\right\}
\end{aligned}
$$

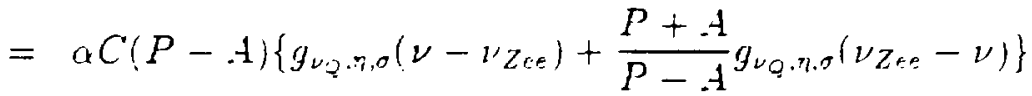

$$
\begin{aligned}
& ={ }_{\alpha} C(P-A)\left\{g_{\nu_{Q}, \eta, \sigma}\left(\nu-\nu^{\prime} Z_{\epsilon \epsilon}\right)+\frac{1+A / P}{1-A / P} g_{\nu_{g}, \eta, \sigma}\left(\nu_{Z \epsilon \epsilon}-\nu j\right\}\right. \text {. }
\end{aligned}
$$

With this form. we see explicitly how $\chi^{\prime \prime}$ depends on the material parameters $\nu_{Q} . \eta, \sigma$. the polarizations $A$ and $P$, and the proportionality $\alpha$. With (4.3). (4.2), and (4.1). I is described in terms of these same parameters. For a given value of $L_{0}$, the behavior of any circuit which contains the rf coil can be computed using ac circuit analysis. Through the dependence of $L$, this behavior is parameterized by $\nu_{Q}, \eta, \sigma, A$. $P$. and $\alpha$. We can thus compute the manner in which a circuit responds to these parameters. and by comparison with a measured circuit response, determine the actual values of the parameters. We term this procedure curve fitting. Because of its complexity. it is necessarily implemented on a computer.

For curve fitting, it is useful to introduce the variables

$$
\begin{aligned}
C^{\prime} & \equiv \alpha C(P-A) \\
r^{\prime} & \equiv \frac{1+A / P}{1-A / P} .
\end{aligned}
$$

These equations can be inverted to give

$$
\begin{aligned}
& A=\frac{C^{\prime}}{a C^{\prime}} \frac{r^{\prime}-1}{2} \\
& P=\frac{C^{\prime}}{{ }_{\alpha} C^{\prime}} \frac{r^{\prime}+1}{2} .
\end{aligned}
$$


U'sing (4.6) $\chi^{\prime \prime}(\omega)$ can then be expressed

$$
\lambda^{\prime \prime}(\omega)=C^{\prime}\left\{g_{\nu_{Q}, \eta, \sigma}\left(\nu-\nu_{Z_{\epsilon e}}\right)+r^{\prime} g_{\nu_{Q}, \eta, \sigma}\left(\nu_{Z e e}-\nu\right)\right\}
$$

With this parameterization of $\chi^{\prime \prime}$, we can use (4.3), (4.2), and (4.1) to parameterize $L$ in terms of $\nu_{Q}, \eta, \sigma, c^{\prime}$, and $r^{\prime}$.

We note that because $\alpha$ is unknown, we cannot use $C^{\prime}$ and $r^{\prime}$ in (4.8) to determine $A$ and $P$. However from (4.8), the ratio

$$
A / P=\frac{r^{\prime}-1}{r^{\prime}+1}
$$

is independent of $\alpha$. Thus, by itself the curve fitting procedure only determines the ratio $A / P$. To go beyond this we need additional information. Below we discuss two methods for obtaining it: the thermal equilibrium (TE) method, and the spin temperature hypothesis.

\subsection{Thermal Equilibrium Method}

In general, the response of an NMR circuit depends through $L$ on both $\chi^{\prime}(\omega)$ and $\chi^{\prime \prime}(\omega)$. The circuit is usually designed, however, to suppress dependence on $\chi^{\prime}(\omega)$, and to be as nearly as possible linear in $\chi^{\prime \prime}(\omega)$. Writing $V_{s i g}$ for our measured signal, and $\beta$ for a proportionality constant, we have

$$
\begin{aligned}
V_{s i g}(\omega) & \approx \beta_{\chi}^{\prime \prime}(\omega) \\
& =\beta a C\left\{(P-A) g_{\nu_{Q}, \eta, \sigma}\left(\nu-\nu_{Z e e}\right)+(P+A) g_{\nu_{Q}, \eta, \sigma}\left(\nu_{Z e e}-\nu\right)\right\}
\end{aligned}
$$

Integration gives

$$
\int_{0}^{\infty} V_{s i g}(\omega) d \omega=2 \beta \alpha C P
$$


The constant $2,3 \alpha C$ is measureri by placing the target material in a knrout state of polarization. This is done by allowing the material to come ro thermin?

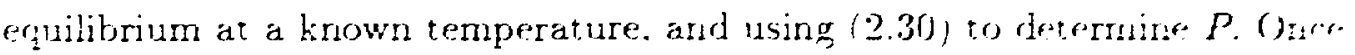
determined in this way $2.3 \times C$ ma be used with $4.11 \mathrm{j}$ to determine $P$, itm with enhanced polarization. When used in corijunction with the $A / P$ wn: from curve fitting. this method gives indeperdent measurements of $A$ and $P$.

The principle difficuly with the TE roethod is that at available fields and temperatures. the TE polarization is small. The large amplifice tion needed to record the XMR signal contributes noise which ards wrerttairty to the integral in $(4.11$ ). Other complications are norlinearities arri the contribution of $\chi^{\prime}(\omega)$. Later in this chapter we consider these cffects for clar particular circuit.

\subsection{Spin Temperature Method}

It is widely believed [Del iffe] that under circumstances where energy wel populations in the deuteron ensemble have reached a steady state. hif popmlarion $n(E)$ mist take the form

$$
\operatorname{ri}(E)=. l^{-\frac{E}{k r}}
$$

where $E$ is energy, $k$ is the Bohtzmann constant. I' is a normalizatiora ros. stent. and $\tau$ is a parameter. Though this form is familiar from the staticical

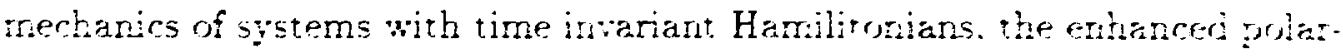
ization of the target tesults from the arplication of a strong microware seld. 
Results from statistical mechanics do not necessarily apply. The assumed validity of (4.12) is known as the spin temperature hypothesis. The parameter $\tau$ is called the spin temperature, and may take both positive and negative values.

For the spin one target of interest here, the quadrupole splitting is snall compared to the Zeeman splitting, so we can ignore the quadrupole contribution when computing populations. The Zeeman Hamiltonian gives three equally spaced energy levels, so from (4.12)

$$
\frac{n\left(E_{+}\right)}{n\left(E_{0}\right)}=\epsilon^{-\frac{\Delta E}{k r}}=\frac{n\left(E_{0}\right)}{n\left(E_{-}\right)} \equiv r
$$

Thus (2.17) takes the form (2.23). From reasoning identical to that which lead to (2.24) and (2.25), we obtain

$$
\begin{aligned}
& P=\frac{r^{2}-1}{r^{2}+r+1} \\
& A=\frac{r^{2}-2 r+1}{r^{2}+r+1} .
\end{aligned}
$$

Thus

$$
A / P=\frac{r-1}{r+1}
$$

Comparing this to $(4.10)$, we see

$$
r=r^{\prime}
$$

where $r^{\prime}$ is the fitting parameter in (4.9). Thus, with the spin temperature assumption, curve fitting alone can determine $A$ and $P$ via

$$
P=\frac{r^{\prime 2}-1}{r^{\prime 2}+r^{\prime}+1}
$$


and

$$
A=\frac{r^{\prime 2}-2 r^{\prime}+1}{r^{\prime 2}+r^{\prime \prime}+1}
$$

We emphasize that when the spin temperature assumption is valid, $A$ and $P$ are related by (2.28), and thus polarizations are restricted to the solid line in Fig. 2.2. Clearly the spin temperature assumption is not valid in experiments where alignment is enhanced by hole burning or other methods.

\subsection{Numerical Methods}

To implement the fitting procedure we seek computationally efficient computer routines which can numerically evaluate $I_{\nu Q, n, \sigma}$ and its Hilbert transform to obtain $\chi^{\prime \prime}$ and $\chi^{\prime}$. This process involves the evaluation of several integrals. Since these integrations appear to be analytically intractable we must resort to numerical methods. $I_{\nu_{Q}, \eta, \sigma}$ was defined in the previous chapter as an integral which involved the function $K$, where $K$ itself is defined as an integral. The Hilbert transformation required to determine $\chi^{\prime}$ involves another integration. The number of computer steps needed for numerical integration of it general integrand whose values are specified at $n$ points grows as $n^{2}$. but because of the special forms which are taken by our integrals, we can reduce this number to $n \log n$.

We may eliminate the integral which defines $I_{i}$ with the following approximation.

$$
H(x) \cong a_{0}+a_{1}(1-x)+a_{2}(1-x)^{2}
$$




$$
-\left(b_{0}+b_{1}(1-x)+b_{2}(1-x)^{2}\right) \ln (1-x)
$$

where

$$
\begin{array}{ll}
a_{0}=1.3862944 & b_{0}=0.5 \\
a_{1}=0.1119723 & b_{1}=0.1213478 \\
a_{2}=0.0725296 & b_{2}=0.0288729 .
\end{array}
$$

The absolute difference between the true value of $K^{\prime}(x)$ and the approximation is less than $3 \times 10^{-5}$ for all $x$ which satisfy $0 \leq x<1$ [AS-1]. Note that this accuracy is maintained even as $x \rightarrow 1$, where both $K^{*}$ and the logarithm term in the approximation become infinite. A computer algorithm for generating $K$ on the closed interval $[0,1]$ may then use the approximation on $[0,1)$ and ret urn the largest floating point number available for $K^{2}(1)$. From the previous chapter, we see that with such an algorithm for $K$, only square roots and polynomials are needed to construct an algorithm for $f_{\eta}$.

The next step in the numerical evaluation of $I_{\nu_{Q}, \eta, \sigma}$ is to compute $g_{\nu_{Q}, n, \sigma}$. Its form as a convolution with the function $f_{n}\left(\frac{2 z}{\nu_{Q}}\right)$ suggests that we use the convolution theorem ${ }^{2}$ to evaluate the integral, i.e.,

$$
\begin{aligned}
\tilde{g}_{\nu_{Q}, \eta, \sigma}(\omega) & =\frac{2}{\nu_{Q}}\left(f_{\eta}\left(\frac{2 z}{\nu_{Q}}\right)\right) \tilde{\delta_{\sigma}}(\omega) \\
& =\frac{2}{\nu_{Q}}\left(\frac{\nu_{Q}}{2} \tilde{f}_{\eta}\left(\frac{\nu_{Q} \omega}{2}\right)\right) \tilde{\delta_{\sigma}}(\omega) \\
& =\tilde{f}_{\eta}\left(\frac{\nu_{Q}}{2} \omega\right) \tilde{\delta_{\sigma}}(\omega) .
\end{aligned}
$$

\footnotetext{
${ }^{2}$ See appendix $B$
} 
We then have

$$
g_{\nu Q, \eta, \sigma}(x)=\mathcal{F}^{-1}\left[\tilde{g}_{\nu_{Q}, \eta, \sigma}\right](x),
$$

where $\tilde{\delta}_{\sigma}$ is found analytically to be ${ }^{3}$

$$
\tilde{\delta}_{\sigma}=e^{-\frac{z^{2} w z}{2}},
$$

and $\tilde{f}_{n}$ is evaluated numerically with a fast Fourier transform (EFT) routine. (We have not found a useful analytic form for $\tilde{f}_{\eta}$.) Thus two FFT's and a simple product replace the need to compute a numerical integral in the evaluation of $g_{\nu_{Q}, \eta, \sigma}$. The computation time for FFT's grows only as $n \log n$ [Kin], rather than the $n^{2}$ associated with numerical integration. Another advantage is that the FFT algorithm tolerates the singularity in $f_{n}$ well, so that nothing special need be done to accommodate it. This is in contrast to using a direct numerical integration [Sp].

Having evaluated $g_{\nu_{Q}, \eta, \sigma}$ numerically, a simple linear combination gives us the desired $I_{\nu_{Q}, \eta, \tau}$, which is proportional to $\chi^{\prime \prime}$. We must now use (4.3) to obtain $\chi^{\prime}$. If we ignore the $\mathcal{P}$ in (4.3) it appears to be a convolution of the functions $\frac{1}{\nu}$ and $\frac{\chi^{\prime \prime}(\mu)}{\pi}$. In the theory of generalized functions [Li] we are indeed allowed to drop the $\mathcal{P}$ if we treat $\frac{1}{x}$ as a generalized function. The generalized inverse Fourier transform of $\frac{1}{x}$ is found to be [ $\mathrm{Li}-1$ ]

$$
\mathcal{F}^{-1}\left[\frac{1}{\omega}\right](t)=\left\{\begin{array}{cc}
\frac{i}{2} & t>0 \\
\frac{-i}{2} & t<0
\end{array}\right.
$$

\footnotetext{
${ }^{3}$ The Lorentzian form of $\delta_{\sigma}$ also has a simple transform. See appendix B.
} 
The convolution theorem in its inverse form ${ }^{4}$ may be applied in the same manner as before to obtain

$$
\begin{aligned}
\gamma^{\prime}(\omega) & =-\frac{1}{\pi} \int_{-\infty}^{\infty} \frac{\chi^{\prime \prime}\left(\omega^{\prime}\right)}{\omega-\omega^{\prime}} d \omega^{\prime} \\
& =-\frac{1}{\pi} 2 \pi \mathcal{F}\left[\mathcal{F}^{-1}\left[\chi^{\prime \prime}\right] \times \mathcal{F}^{-1}\left[\frac{1}{\omega}\right]\right](\omega) \\
& =-2 \mathcal{F}\left[\mathcal{F}^{-1}\left[\chi^{\prime \prime}\right] \times\left\{\begin{array}{cc}
\frac{i}{2} & t>0 \\
\frac{-i}{2} & t<0 .
\end{array}\right](\omega)\right. \\
& =\mathcal{F}\left[\mathcal{F}^{-1}\left[\chi^{\prime \prime}\right] \times\left\{\begin{array}{cc}
-i & t>0 \\
i & t<0 .
\end{array}\right](\omega) .\right.
\end{aligned}
$$

Once again two FFT's replace a numerical integration. Note also that there are no singularities in (4.14) where as conventional numerical integration requires special handling of the singularity at $\omega=\omega^{\prime}[\mathrm{Sp}]$.

With $\chi^{\prime}$ and $\chi^{\prime \prime}$ thus determined up to a normalization constant, in terms of $\nu_{Q}, \eta, \sigma, P$, and $A$, we may use the value $L=L_{0}(1+4 \pi)$ in a computer model of the circuit, and adjust these constants until a good match between measured data and computer model is achieved.

\subsection{Circuit Model}

The NMR coil is wound around the target material and located inside the mixing chamber of our dilution refrigerator. In our apparatus it is connected

\footnotetext{
${ }^{4}$ See appendix B
} 


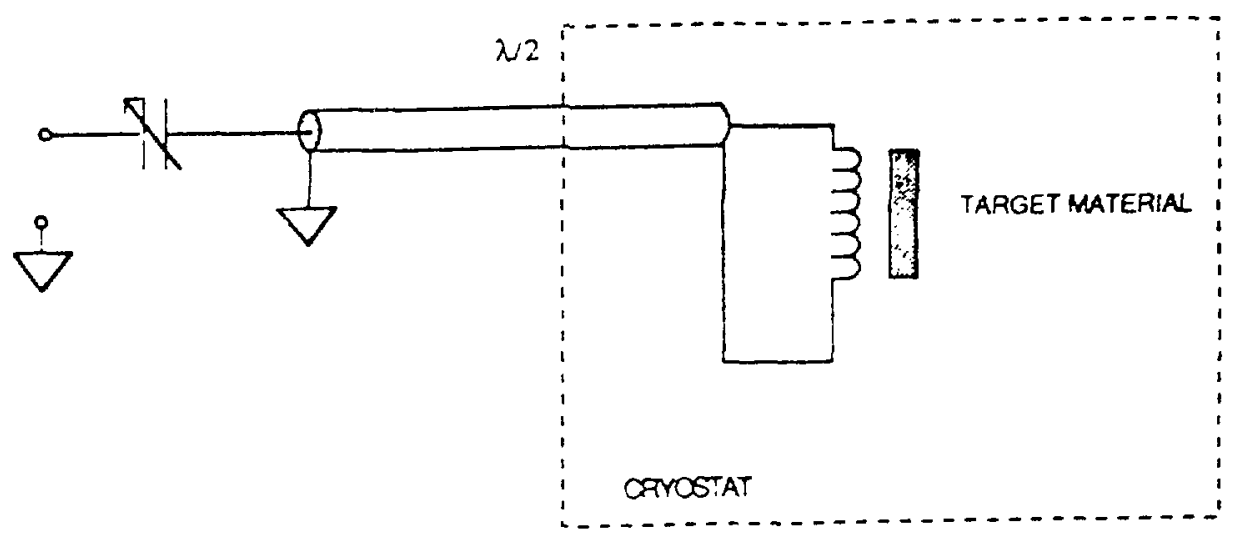

Figure 4.1: Schematic representation of connection to NMR coil.

to the outside orld with a two ineter length of beryllinm-copper coaxial cable. ${ }^{5}$ Fig. 4.1 shows the configuration. From circuit theory [Ra], we know the impedance of this arrangement is given by

$$
Z_{\text {sig }} \equiv \frac{-i}{\omega C}+Z_{c a b}
$$

where

$$
Z_{c a b} \equiv Z_{0} \frac{(R+i \omega L)+Z_{0} \tanh (l \gamma)}{Z_{0}+(R+i \omega L) \tanh (l \gamma)}
$$

\footnotetext{
${ }^{5}$ Uniform Tubes, Inc. Microcoax part no. UT-T-85-50-B-B.
} 
Table 4.1: Cable parameters at various frequencies and temperatures.

$\begin{array}{cccc}\text { Freq. (MHz) } & \text { Temp. (K) } & \alpha(\mathrm{dB} / \mathrm{m}) & \mathrm{R}(\Omega / \mathrm{m}) \\ 16 & 77 & 0.0185 & 0.925 \\ 16 & 300 & 0.0328 & 1.64 \\ 100 & 7 \tau & 0.0617 & 3.09 \\ 100 & 300 & 0.0869 & 4.35\end{array}$

and $L$ is the coil inductance given by (4.1), $R$ is the coil resistance. $l$ is the cable length,

$$
\gamma \equiv \sqrt{(\mathcal{R}+i \omega \mathcal{L})(\mathcal{G}+i \omega \mathcal{C})}
$$

and

$$
Z_{0} \equiv \sqrt{\frac{(\mathcal{R}+i \omega \mathcal{L})}{(\mathcal{G}+i \omega \mathcal{C})}}
$$

$\gamma$ and $Z_{0}$ are known respectively as the propagation constant and the characteristic impedance. Clearly $Z_{\text {sig }}$ has a complicated dependence on frequency since $L, \gamma, Z_{0}$ all depend on $\omega$. The quantities $\mathcal{C}, \mathcal{L}, \mathcal{R}, \mathcal{G}$ are respectively the capacitance, inductance, resistance, and condurtance, per unit length of the cable. $\gamma$ and $Z_{0}$ are in general, complex quantities. $\mathcal{G}$ is essentially zero. With a network analyzer we measured the propagation speed and the attenuation coefficient for the cable. The propagation speed was found to be $205,734.000$ $\mathrm{m} /$ second. The attenuation results are shown in table (4.1). The quantity $\alpha$ is defined by

$$
\frac{V_{\text {out }}}{V_{\text {in }}}=e^{-a l} \text {. }
$$

Since we operate at $16 \mathrm{MHz}$ and half the cable is inside the cryostat, we take $a=0.925 \Omega / \mathrm{m}$. To relate these resilts to $\mathcal{C}, \mathcal{L} . \mathcal{R}$, we assume that $Z_{0} \approx 50 \Omega$. 
We then use $\alpha=\frac{R}{2 Z_{0}}[\mathrm{Ra}-1]$ to obtain $\mathcal{R}=2 Z_{0} \alpha=0.925 \Omega / \mathrm{m}$. By allowing $\mathcal{R}$ to vary in the computer fitting procedure we found that $\mathcal{R}=0.70 \Omega / \mathrm{m}$ gave the best fit. This was considered to be reasonable because of the low temperatures inside the cryostat.

By neglecting $\mathcal{R}$ and $\mathcal{G}$ in (4.18), we obtain

$$
Z_{0}=\sqrt{\frac{\mathcal{L}}{\mathcal{C}}}
$$

The propagation velocity is given by $[\mathrm{Ra}]$

$$
v=\frac{1}{\sqrt{\mathcal{L C}}}
$$

The manufacturer guarantees that $Z_{0} \approx 50 \Omega$, so with our measured value of l', we can obtain $\mathcal{L}$ and $\mathcal{C}$. Our results are summarized by

$$
\begin{aligned}
\mathcal{C} & =97.21 \mathrm{pF} / \mathrm{m} \\
\mathcal{L} & =0.244 \mu \mathrm{H} / \mathrm{m} \\
\mathcal{R} & =0.70 \Omega / \mathrm{m} \\
\mathcal{G} & =0.0 \mathrm{mho} / \mathrm{m} .
\end{aligned}
$$

With these constants fixed, we sce that our intended measurement of $P$ and $A$ depends on the manner in which they affect $Z_{c a b}$. This dependence is quite small, so a balanced circuit technique is indicated. As nearly as possible, an identical copy of the target coil and cable is constructed, but the material inside this dummy coil is teflon, which will not be polarized under our experimental conditions. The dummy coil is also placed in the mixing chamber of the cryostat. By amplifying only the difference between 
the signal arm and the dummy arm we avoid saturating the amplifiers with features of the signal which are independent of polarization. This subtraction is accomplished with a $180^{\circ}$ radio frequency hybrid [No].

To model the behavior of the hybrid circuit we use the four-port $\mathrm{S}$-parameter description $[\mathrm{No}]$. With this method, the relationship between amplitudes of incident and reflected voltage waves is described by a matrix equation. In electrical engineering literature, this matrix is called an S-matrix. Voltages in the cable connected to the device are written

$$
V_{3}=\left(h_{3} \epsilon^{-i k x}+a_{3} e^{i k x}\right) \epsilon^{i \omega t},
$$

where distance $x$ is zero at the device connection and increases with the distance from the connection. Thus $a_{j}$ and $b_{j}$ are respectively, the amplitudes for the incident and reflected waves. The subscript $j$ indexes the different ports. These conventions are shown in Fig. 4.2.

Current in the cable takes the form

$$
I=\frac{1}{Z_{0}}\left(b, e^{-i k x}-a_{j} e^{i k x}\right) \epsilon^{i \psi t} .
$$

For the $180^{\circ}$ hybrid circuit, the S-matrix equation is given by [No]

$$
\left(\begin{array}{l}
b_{1} \\
b_{2} \\
b_{3} \\
b_{4}
\end{array}\right)=\frac{\epsilon^{i \theta(\omega)}}{\sqrt{2}}\left(\begin{array}{cccc}
0 & 0 & 1 & 1 \\
0 & 0 & -1 & 1 \\
1 & -1 & 0 & 0 \\
1 & 1 & 0 & 0
\end{array}\right)\left(\begin{array}{l}
a_{1} \\
a_{2} \\
a_{3} \\
a_{4}
\end{array}\right) .
$$

To find how the output voltage. $b_{2}$, depends on $Z_{s i g}$ as we scan the frequency, we assume that $a_{1}$ is constant with changes in the driving frequency, and that 


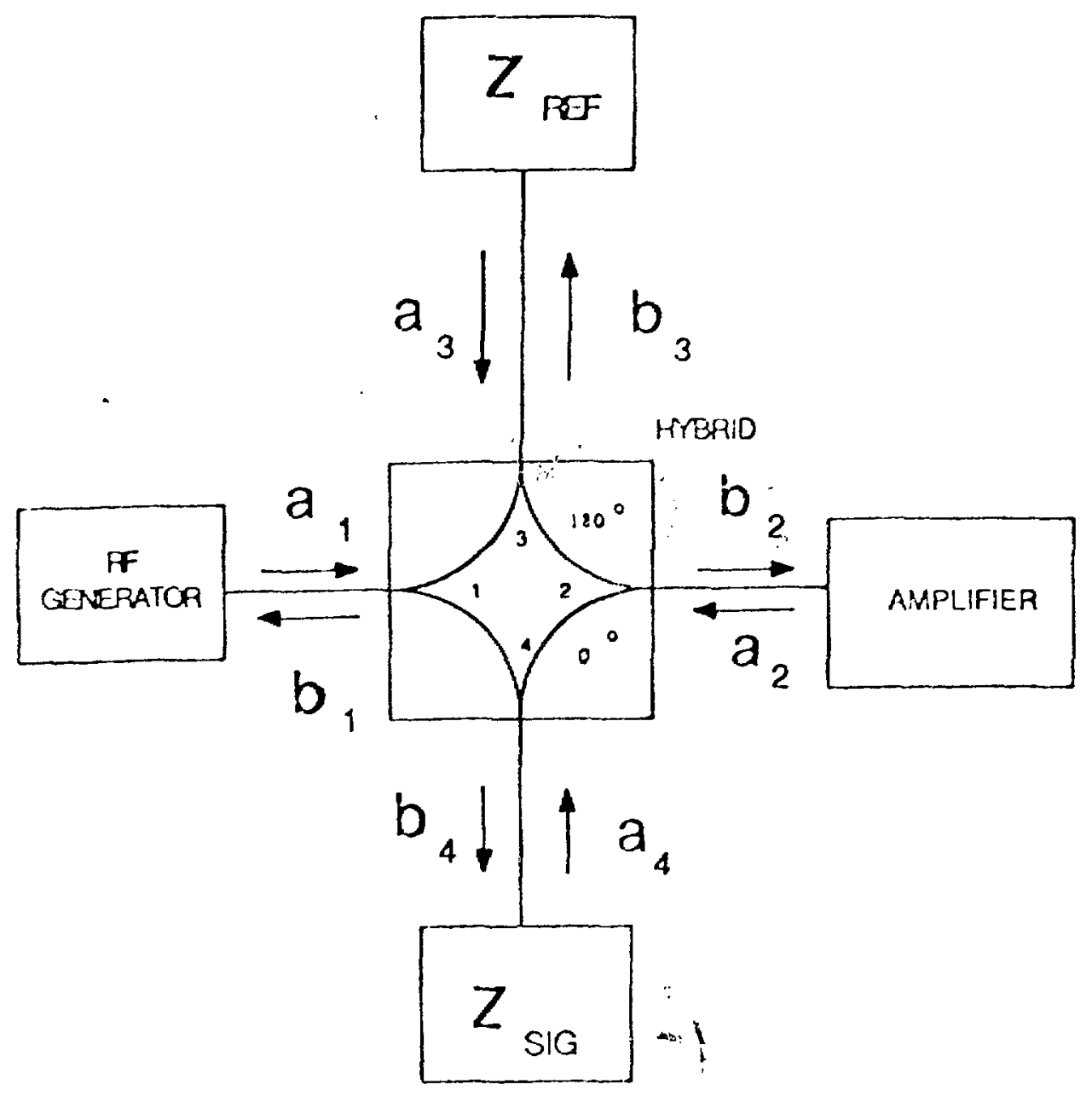

Figure 4.2: Voltage wave coriventions for $180^{\circ}$ hybrid. 
$a_{2}=0$ (i.e., no reflection occurs at the output amplifier). Equation (4.19) gives

$$
\begin{aligned}
& b_{3}=\frac{a_{1}}{\sqrt{2}} e^{i \theta} \\
& b_{4}=\frac{a_{1}}{\sqrt{2}} e^{i \theta} .
\end{aligned}
$$

From the definition of impedance, we know

$$
\begin{aligned}
Z_{s i g} & =\frac{V_{4}(x=0)}{I_{4}(x=0)} \\
& =\frac{b_{4}+a_{4}}{\left(b_{1}-a_{4}\right) / Z_{0}} \\
& =Z_{0} \frac{\frac{a}{\sqrt{2}} e^{i \theta}+a_{4}}{\frac{a_{1}}{\sqrt{2}} e^{i \theta}-a_{4}}
\end{aligned}
$$

Solving for $a_{4}$ we obtain

$$
a_{4}=\frac{Z_{s i g}-Z_{0}}{Z_{s i g}+Z_{0}} \frac{a_{1}}{\sqrt{2}} e^{i \theta} .
$$

Similarly for th:e reference arm we have

$$
a_{3}=\frac{Z_{\text {ref }}-Z_{0}}{Z_{\text {ref }}+Z_{0}} \frac{a_{1}}{\sqrt{2}} e^{i \theta} .
$$

The S-matrix then gives

$$
\begin{aligned}
b_{2} & =\frac{e^{i \theta}}{\sqrt{2}}\left(a_{4}-a 3\right) \\
& =\frac{e^{2 i \theta}}{\sqrt{2}} a_{1}\left(\frac{Z_{\text {sig }}-Z_{0}}{Z_{\text {sig }}+Z_{0}}-\frac{Z_{\text {ref }}-Z_{0}}{Z_{\text {ref }}+Z_{0}}\right) \\
& =e^{2 i \theta} a_{1} Z_{0} \frac{Z_{\text {sig }}-Z_{\text {ref }}}{\left(Z_{\text {sig }}+Z_{0}\right)\left(Z_{\text {ref }}+Z_{0}\right)} .
\end{aligned}
$$

Writing $V_{\text {in }}$ for $a_{1}$ and $V_{\text {out }}$ for $b_{2}$, we have

$$
\frac{V_{\text {out }}}{V_{\text {in }}}=e^{2 i \theta} Z_{0} \frac{Z_{\text {sig }}-Z_{\text {ref }}}{\left(Z_{\text {sig }}+Z_{0}\right)\left(Z_{\text {ref }}+Z_{0}\right)} .
$$


$Z_{s, y}$ is defined in (4.15) and $Z_{\text {ref }}$ has a similar form given by

$$
Z_{r \epsilon f} \equiv \frac{-i}{\omega C_{r}}+Z_{0} \frac{\left(R_{r}+i \omega L_{r}\right)+Z_{0} t \operatorname{anh}\left(l_{r}\right)}{Z_{0}+\left(R_{r}+i \omega L_{r}\right) \tanh \left(l_{r}\right)}
$$

where symbols are defined as in (4.15) and the $r$ subseript indicates a quantity which pertains to the reference arm. $L_{r}$ has no frequency dependence ince the corresponding coil does not contain polarized deuterons.

Though ideally we want $l_{r}=l, R_{r}=R, C_{r}=C$, and $L_{r}=L_{0}$. in practice there is some discrepancy. By allowing these parameters to vary while using the fitting algorithm to match data from an unpolarized sample $(x=0)$, we found that very good fits could be achieved. We then left these parameters fixed while making fits to polarized clata.

We note that (4.20) shows $V_{\text {out }} / V_{\text {in }}$ to be a nonlinear function of $(\omega)$. In principle, this means that a fitting procedure should use the true physical value of $V_{u u t} / V_{\text {in }}$ to determine $\chi(\omega)$. This means the fit does not scale: if $\chi$ is the best fit for the data $\frac{V_{\text {ans }}}{V_{i n}}$, then for some constant a. ax may not be the best fit for the data $\alpha \frac{V_{2 u}}{V_{i n}}$. In other words, the (relative) slape of (4.20) changes with the magnitude of $\chi$. Numerical sinulations, however, have shown this effect to be insignificant.

\subsection{Approximations}

Normally $\chi$ is expected to be small enough for $(4.20)$ to be well represented by a first order Taylor expansion in $\chi$. In this case. $\frac{l_{\text {aus }}}{V_{i n}}$ is linear in $\downarrow$ and the considerations of the last paragraph are mute. For computer work there 
is no reason not to use (4.20), but for some purposes, it is useful to linearize (4.20) and more clearly expose the role of $x$. To this end. we expand $(4.20)$ in a first order Taylor series in $\gamma$, and obtain

$$
\frac{V_{\text {out }}}{\Gamma_{\text {in }}} \approx \epsilon^{2, \theta} Z_{0}\left(\frac{Z_{s i g}(\chi=0)-Z_{r t f}}{\left(Z_{s i g}(\chi=0)+Z_{0}\right)\left(Z_{r \in f}+Z_{0}\right)}+\frac{\chi \partial_{\chi} Z_{s i g}(\chi=0)}{\left(Z_{s i g}(\chi=0)+Z_{0}\right)^{2}}\right) .
$$

The partial derivative may be analytically evaluated and gives

$$
\partial_{\chi} Z_{s i g}(\chi=0)=4 \pi i Z_{0}^{2} \omega L_{0} \frac{1-\tanh ^{2}\left(i_{\gamma}\right)}{\left(Z_{0}+\left(R+i \omega L_{0}\right) \tanh \left(l_{\gamma}\right)\right)^{2}} .
$$

We define the background $b(\omega)$ as

$$
b(\omega) \equiv Z_{0} \frac{Z_{s t g}(\chi=0)-Z_{r e f}}{\left(Z_{s i g}(\chi=0)+Z_{0}\right)\left(Z_{r e f}+Z_{0}\right)} .
$$

$b(\omega)$ depends on $\omega$ but not on $\backslash$, and clearly for the ideal case of identical signal and reference arms $b(\omega)=0$.

We define $h(\omega)$ to be $\frac{1}{3}$ times the coefficient multiplying x. thus

$$
h(\omega) \equiv \frac{4 \pi Z_{0}^{3} \omega L_{0}}{\left(Z_{s i g}(\chi=0)+Z_{0}\right)^{2}} \frac{1-\tanh ^{2}(l \gamma)}{\left(Z_{0}+\left(R+i \omega L_{0}\right) \tanh (l \gamma)\right)^{2}},
$$

and we have

$$
\frac{V_{\text {out }}}{V_{\text {in }}} \approx e^{2 i \theta}(i h(\omega) \chi(\omega)+b(\omega)),
$$

In general $h(\omega), \gamma(\omega)$, and $b(\omega)$ are all complex.

We further simplify by assuming the cable is perfect $(\mathcal{G}=\mathcal{R}=0)$. This gives $\gamma=i \omega \sqrt{\mathcal{L C}}$, which is purely imaginary and $Z_{0}=\sqrt{\frac{c}{c}}$. We can then use a property of $\tanh$ to write $\tanh (i \omega / \sqrt{\mathcal{L C}})=i \tan (\omega l \sqrt{\mathcal{L C}})$. We also assume our inductor has negligible resistance, and thus $R=0$. Finally we 
assume the capacitors $C^{\prime}$ and $C_{r}$ have been chosen to make $Z_{s 13}$ and $Z_{r e}$ real. and that this remains approximately true for the frequency range of interest.

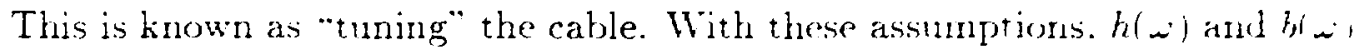
become real. th takes the form

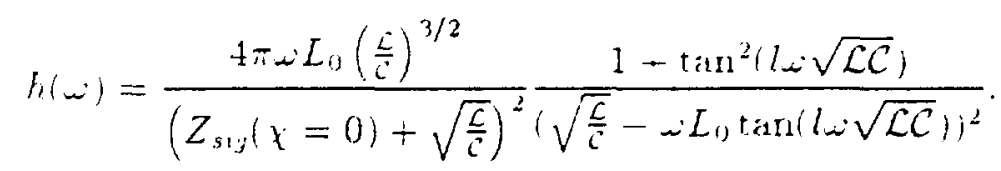

and we have

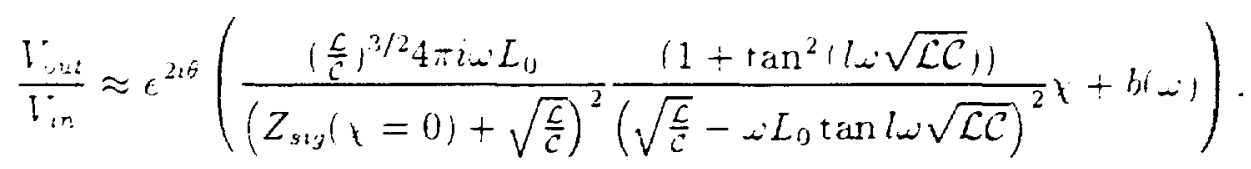

We will see later that the $e^{22 \theta}$ factor is unimportant, so for now we trop it and write

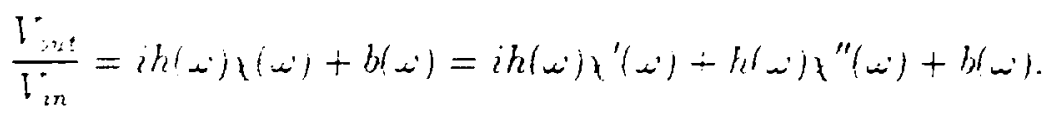

This expression demonstrates that if our detector is of a type which detects only the real part of $l_{\text {uut }}$, the signal will be proportional to $h(\omega) x^{\prime \prime}(\omega)+b(\nu$ ! and will be independent of $\chi^{\prime}(\omega)$. This approximate result motivates our 11se of the homodyne detection system, which as we now show, detects only the real part.

\subsection{Homodyne Detector}

Fig. 4.3 gives a schematic representation of a homodyne detector. The rf generator produces a voltage $V e^{i+t}$. The device $A$. multiplies $l e^{1+t}$ by a complex 


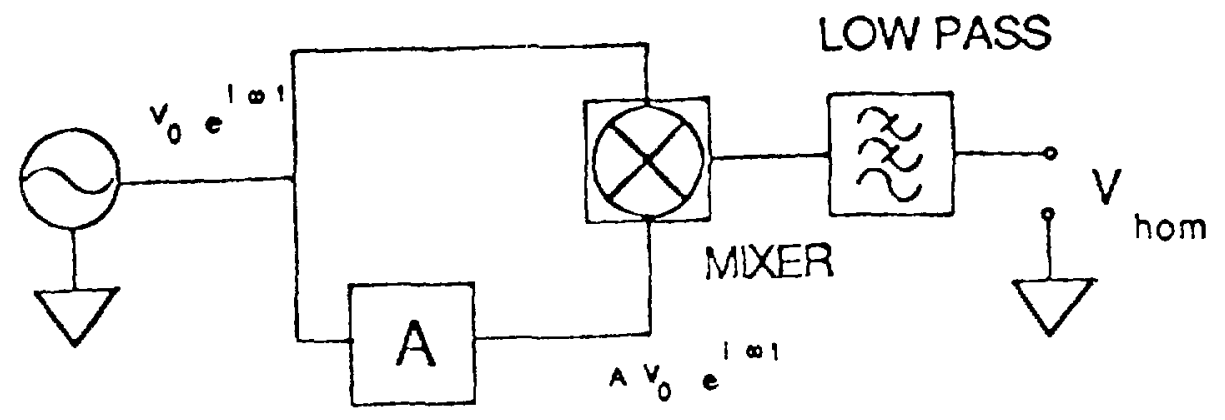

Figure 4.3: Homodyne detector.

number which is modulated by the signal of interest. In our case (4.24) gives

$$
A \approx i h(\omega) \chi^{\prime}(\omega)+h(\omega) \chi^{\prime \prime}(\omega)+b(\omega) .
$$

The output of the mixer is the product of the two input signals. Thus

$$
\begin{aligned}
V_{\text {mix }} & =\left(\operatorname{Re} A V_{0} e^{i \omega t}\right)\left(\operatorname{Re} V_{0} e^{i \omega t}\right) \\
& =\left(\operatorname{Re} A V_{0} \cos \omega t-\operatorname{Im} A V_{0} \sin \omega t\right)\left(V_{0} \cos \omega t\right) \\
& =V_{0}^{2}\left(\operatorname{Re} A \cos ^{2} \omega t-I m A \sin \omega t \cos \omega t\right) \\
& =V_{0}^{2}\left(\frac{\operatorname{Re} A}{2}(1+\cos 2 \omega t)-\frac{\operatorname{Im} A}{2} \sin 2 \omega t\right) \\
& =\frac{V_{0}^{2}}{2} \operatorname{Re} A+\frac{V_{0}^{2}}{2}(\operatorname{Re} A \cos 2 \omega t-\operatorname{Im} A \sin 2 \omega t) .
\end{aligned}
$$

By low-pass filtering $V_{\text {mix }}$ we eliminate the second and third torms, and we obtain

$$
V_{\text {hom }}=\frac{V_{0}^{2}}{2} \operatorname{Re} A
$$


With (4.24) we would have

$$
V_{h o m} \times h(\omega) x^{\prime \prime}(\omega)+b(\omega)
$$

Without the assumptions leading up to (4.24), we cannot expect our circuit to eliminate $\chi^{\prime}(\omega)$ completely: we can expect $\chi^{\prime}(\omega)$ to be strongly suppressed.

Fig. 4.4 shows a schematic of our circuit. The active components introduce phase shifts which must be compensated if the above results are to remain valid. In practice, a variable phase shift device is included in the circuit, and adjusted until the dispersive component of the signal is minimized. This technique also cancels the phase shift due to the $e^{2 i \omega}$ factor which appears in (4.20), and this justifies our dropping $e^{2 i \omega}$ from (4.24).

It is possible that the phase shift is frequency dependent. If this dependency is known, it is straight forward to include it in the computer model. If the frequency dependence is unknown, we can assume that it is linear and use the computer fitting algorithm to choose the best linear function.

Having motivated the use of the homodyne detector with approximations, we now apply it to our exact result (4.20). This gives

$$
V_{\text {hom }} \equiv \frac{\left|V_{i n}\right|^{2}}{2} \operatorname{Re}\left\{e^{2 i \theta} Z_{0} \frac{Z_{\text {sig }}-Z_{\mathrm{ref}}}{\left(Z_{\text {sig }}+Z_{0}\right)\left(Z_{\text {ref }}+Z_{0}\right)}\right\} .
$$

This equation forms the basis of our computer model. The fitting procedure consists of adjusting the various parameters in (4.26) until $V_{\text {hom }}$ matches the measured signal as closely as possible. 


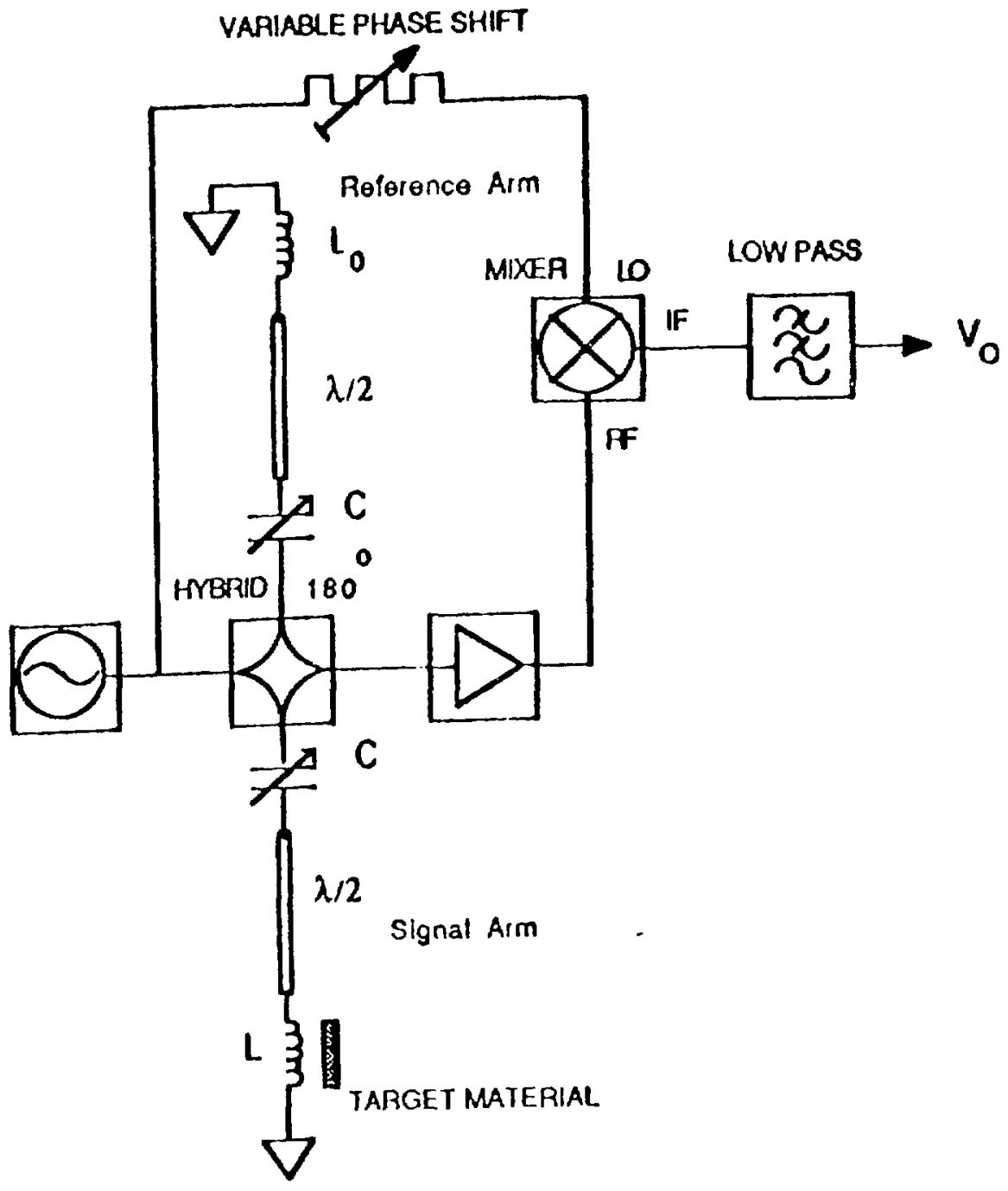

Figure 4.4: Schematic of complete detection circuit. 


\subsection{Computer Algorithm}

Our computer algorithn consists of a straight forward coding of $(4.26)$ inat, the high level computer language, EFL. "Fortran, C, or Pascal would be similarly appropriate. The sarch through parameter space is done 11 sing the subroutine SNLS1, which is part of the CLAMS mathematical library createrd and maintained by Los Alamos National Liboratory. This routine uses the Leverberg-Marquardt algorithm [Mo] to efficiently search parameter spare for a point which minimizes the sum of the sciuared differences between measured MR curves and signals (chi-squared) simulated with (1.26). Routines similar co S.YLS1 are widely available in other mathematical packages. For irstanre. the IMISL [MI] routine LXLSF is similar to SNLS1. The only other library routine used in our program is a fast Fourier transform subroutine. Such routines are also availatsle in IMSL and in numerous other libraries.

As input to our program we use a file which contains on each line a parameter name, its initial value, a symbol indicating whether this parameter will vary during the fit or not, and a comment line describing the parameter. After finding the best set of parameters, the program writes a similar file with these parameter values. This output files is suitable as an input file. and can be easily edited to change values and the set of changeable parameters.

We found the fitting procedure to be sensitive to the initial (i.e.. input) parameter values. That is to say, if the initial parameters are far from

\footnotetext{
"Avajable on most Linix marhines.
} 
their true values, the algorithm may converge to a shape very different from the NMR data. To make good initial guesses we found the formulas given in (3.3), (3.4), and (3.5) to be valuable.

For convenience we summarize the equations from this chapter used to simulate $V_{\text {hom }}$.

$$
\begin{gathered}
V_{h o m}^{\prime} \equiv \frac{\left|V_{i n}\right|^{2}}{2} \operatorname{Re}\left\{e^{2 i \theta} Z_{0} \frac{Z_{s i g}-Z_{r e f}}{\left(Z_{s i g}+Z_{0}\right)\left(Z_{r e f}+Z_{0}\right)}\right\} \\
Z_{r e f} \equiv \frac{-i}{\omega C_{r}^{\prime}}+Z_{0} \frac{\left(R_{r}+i \omega L_{r}\right)+Z_{0} \tanh \left(l_{r} \gamma\right)}{Z_{0}+\left(R_{r}+i \omega L_{r}\right) \tanh \left(l_{r} \gamma\right)} \\
Z_{s i g} \equiv \frac{-i}{\omega C}+Z_{0} \frac{(R+i \omega L)+Z_{0} \tanh \left(l_{\gamma}\right)}{Z_{0}+(R+i \omega L) \tanh \left(l_{\gamma}\right)} \\
\gamma \equiv \sqrt{(\mathcal{R}+i \omega \mathcal{L})(\mathcal{G}+i \omega \mathcal{C})} \\
Z_{0} \equiv \sqrt{\frac{(\mathcal{R}+i \omega \mathcal{L})}{(\mathcal{G}+i \omega \mathcal{C})}} . \\
L=L_{0}(1+4 \pi \chi(\omega)) \\
\chi(\omega)=\chi^{\prime}(\omega)-i \chi^{\prime \prime}(\omega) \\
\chi^{\prime}(\omega)=\frac{-1}{\pi} \mathcal{P} \int_{-\infty}^{\infty} \frac{\chi^{\prime \prime}(x)}{\omega-x} d x \\
\chi^{\prime \prime}(\omega)=C^{\prime}\left\{g_{\nu Q, \eta, \sigma}\left(\nu-\nu_{Z e e}\right)+r^{\prime} g_{\nu Q, \eta, \sigma}\left(\nu_{Z_{e e}}-\nu\right)\right\} .
\end{gathered}
$$

$C$ and $C_{\mathrm{r}}$ represent the capacitance of the tunable capacitors in the signal and reference arms, respectively. $R$ and $R_{r}$ are the NMR coil resistances, and $l$ and $l_{r}$ the cable lengths of the respective arms. $L_{0}$ is the free space inductance of the NMR coil, and $L_{r}$ is the corresponding quantity in the reference arm. The cable parameters $\mathcal{R}, \mathcal{L}, \mathcal{G}$, and $\mathcal{C}$ are respectively, the resistance per meter, inductance per meter, conductance per meter, and capacitance per 
meter. From measurements described earlier in this chapter, $\mathcal{R}, \mathcal{L}, \mathcal{G}$, and $\mathcal{C}$ have known values. $g_{\nu_{Q}, \eta, \sigma}$ is defined in the previous chapter, $C^{\prime}$ is a constant which depends on the packing fraction of the target material (defined page 48 ), and $r^{\prime}$ is related to the polarizations by

$$
A / P=\frac{r^{\prime}-1}{r^{\prime}+1}
$$

The parameter $\theta$ represents the phase shift and is manually adjusted with the variable phase shift (See Fig. 4.4). 


\section{Chapter 5}

\section{Data}

NMR data for E818 was collected with a PDP-11/45 computer and a standalone computer program developed by $\mathrm{D}$. Hill at Argonne National Laboratory [Hi]. This system automatically scans the input frequency (low to high) and digitizes the output of the circuit described in the previous chapter. The frequency is varied from 16.1 to $16.6 \mathrm{MHz}$, and data is recorded at 384 equally spaced intervening points. The scan rate is $17 \mu$ sec per data point, which is judged to be slow enough to justify the use of the constant frequency a.c. circuit analysis used in the previous chapter. A delay of $250 \mu \mathrm{sec}$ is inserted prior to each sweep, to allow transients to decay after the rapid transition from 16.6 to $16.1 \mathrm{MHz}$.

To improve the signal to noise ratio, 1000 sweeps are averaged before any permanent data storage. This takes 6.8 seconds. To keep the signal within range of the 12-bit analogue-to-digital converter (ADC), a signal shaping 
circuit is used between the output of the low pass filter (see Fig. 4.4) and the ADC. The effect of this circuit is to add a quadratic polynomial to the frequency dependence of the homodyne detector. Thus

$$
V_{d: g}(\nu)=V_{h o m}(\nu)+A+B\left(\nu-\nu_{Z_{e t}}\right)+C\left(\nu-\nu^{\prime} Z_{e e}\right)^{2}
$$

where $V_{d i g}$ is the signal which is digitized by the ADC. The constants $A$. $B$, and $C$ are referred to respectively as offset, tilt, and curvature. The curvature is set manually, while offset and tilt are periodically adjusted by the computer. Unfortunately, these constants were not recorded, and thus for fitting purposes they were necessarily left as free parameters.

While monitoring polarization for the p-d scattering experiment, an averaged signal was recorded on floppy disk every five minutes. Once per hour, the magnetic field was changed so the NMR resonance was shifted well outside the frequency sweep. 1000 sweeps were then averaged and recorded on floppy disk as a "baseline" signal. The PDP-11/45 uses this baseline to compute offset and tilt parameters which, in so-far as possible, level the baseline and center it in the range of the ADC. To provide real-time monitoring of the target system, this baseline was subtracted from the signals, and after further background correction with a linear polynomial, was integrated and compared to the TE integrals. These results, though inaccurate, were recorded with the E818 data acquisition system. Later, when accurate TE values had been cietermined off-line, the on-line results were renormalized. In this way, accurate polarization values were determined at five minute intervals throughout E818. 
Fig. 5.1 shows typical data and its corresnondirg baseline. ${ }^{1}$ The distinctive features of $\gamma^{\prime}(\omega)$ (shoulders, peaks, etc.) are easily identified, but are as expected, modified by the circuit effects discussed in the previous chapter. We note in particular, that the highest and lowest $50,000 \mathrm{~Hz}$ in Fig. 5.1 are well outside the NMR resonance (i.e., well past the shoulders of $\chi^{\prime \prime}$ ). In these regions (the wings), we expect the signal to depend only on circuit effects, and to be completely independent of polarization. Initially we had hoped that the baseline data would accurately represent the unpolarized signal and we could use the curve fitting technique on "signal-minus-baseline" data. This would have obviated the need for a detailed model of the circuit. However, comparison of baseline and signal in the wings of Fig. 5.1 shows that the baseline data does not represent the unpolarized signal. This is presumably due to the dependence of the static susceptibility of target material on the magnitude of the static magnetic field, which is different when collecting the baseline data and resonance data. For this reason, it was necessary to develop the circuit model described in the preceding chapter.

The values of $\mathcal{R}, \mathcal{L}, \mathcal{G}, \mathcal{C}, l, l_{r}, R_{r}$, and $R$ (see chapter 4 for notation) were fixed by applying the fitting algorithm to the baseline data. On the scale shown in Fig. 5.1, the modeled baseline was indistinguishable from the data. This gave us confidence that the circuit model was accurate. Quantities which were left free in the fitting algorithm were $r^{\prime}, \eta, \sigma, \nu_{Z e e}, \nu_{Q}, C^{\prime}, \theta, C, C_{r}$, a

\footnotetext{
${ }^{1}$ Signals of this kind are often presented with the baseline subtracted from the signal. We do not follow this practice.
} 


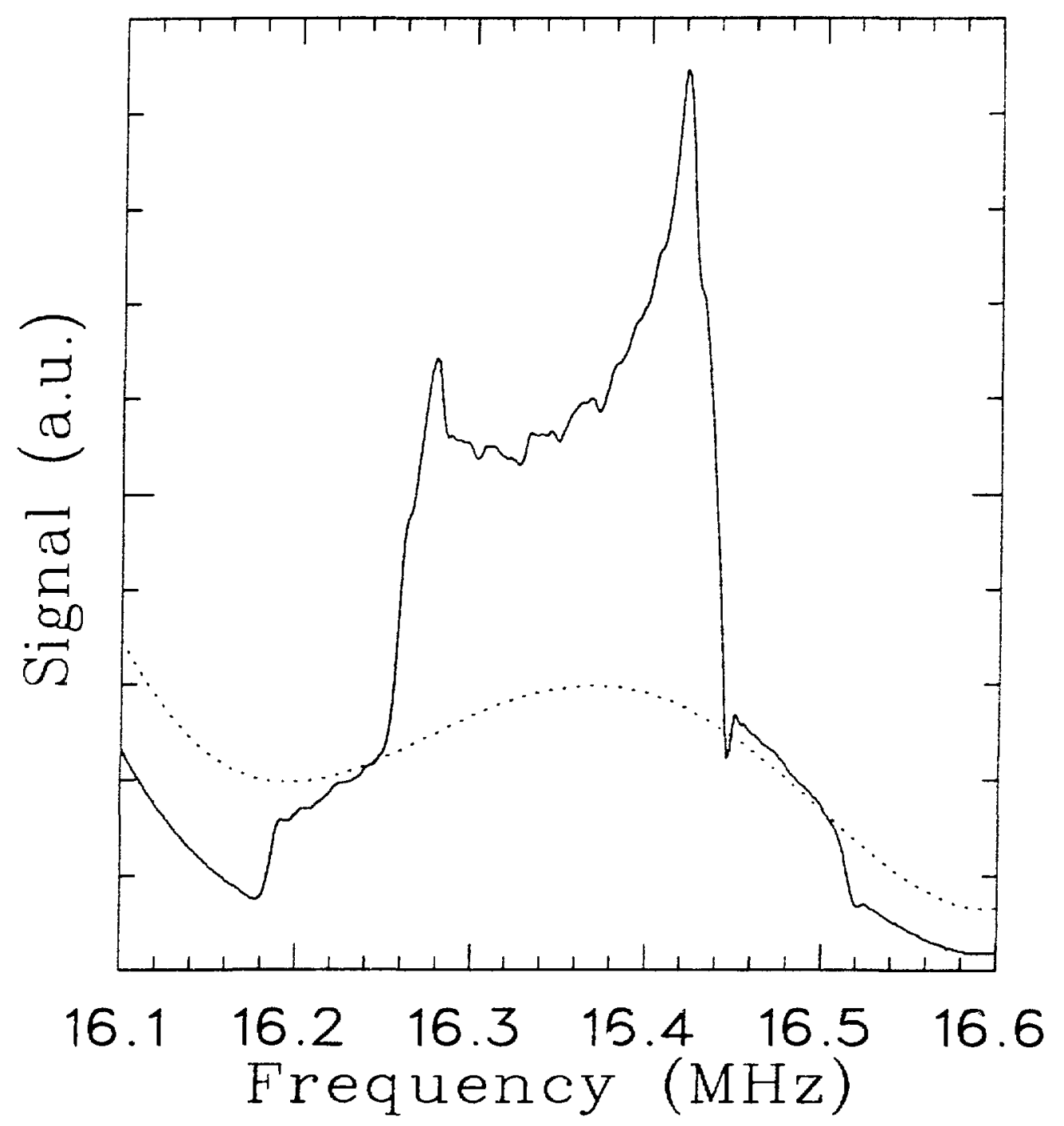

Figure 5.1: Typical signal and baseline data. 
quantity representing the amplifier gain, and three constants representing the unrecorded offset, tilt, and curvature added by the signal shaping circuit mentioned above.

The fitting algorithm was found to be quite sensitive to the initial values of the free parameters. For example, if the initial parameters were sufficiently different from their true values, the algorithm usually found a local minimum for chi-squared which had unreasonable values for $r^{\prime}, \nu_{Q}$, etc. It was therefore necessary for us to choose the initial parameters on a trial and error basis. We felt that if the fit was good in the vicinity of the shoulders and peaks, the parameter value of $r^{\prime}$ would be accurate. Once having obtained a fit satisfying this criterion, we confirmed that the final value of $r^{\prime}$ was independent of the initial value, as long as all other parameters were held fixed.

Fig. 5.2 shows typical data together with the fitted curve found from the model. We note that the model does not fit the data well in the region between the peaks. Here the data exhibits small fluctuations while the model signal is smootk. Though not proven, we conjecture that the reason for this is that the density function $\rho(\theta, \psi)$ given in (3.15) is not completely accirate. In other words, because we have only a finite number of crystal domains, we can expect deviation from the ideal form (3.15). Thus some especially large domain, or some preferred orientation for the chunks of material in the target, might result in a signal with fine structure resembling our daia.

Whatever the reason for the fine structure, its presence explains 


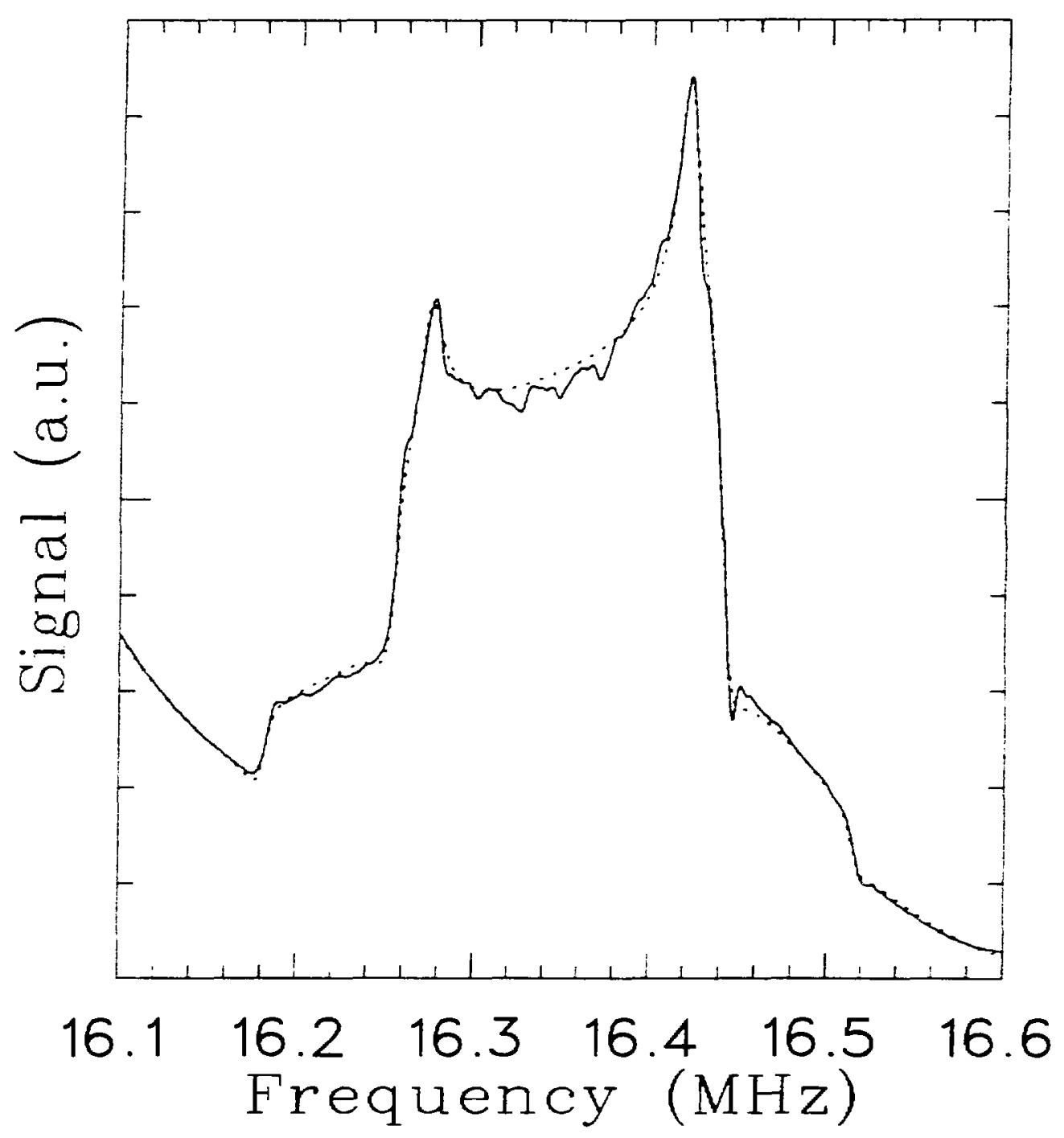

Figure 5.2: Typical signal data (solid) and corresponding model curve (dotted). The fitted curve has $r^{\prime}=1.674(P \approx 33 \%)$. 
why the fitting algorithm is sensitive to the initial parameters. If the model signal is initially within the signal fine structure, chi-squared is minimized for a smooth model curve which roughly bisects each fluctuation in the fine structure. However, this minimization is achieved at the expense of a poor match near the two prominent peaks of the signal. Since we believe that accurate polarizations are obtained only from fits which match well near the peaks and shoulders, we must choose our initial parameterizations well enough that the algorithm does not pull the model curve down inside the fluctuations.

Since we use the curve fitting method to find $r^{\prime}$, it is important to estimate how well this parameter is determined. It is difficult to be mathematically precise in this, since we know we do not model all features of the signal (e.g., the fine structure). To make a qualitative estimate, we plot two model signals in Fig. 5.3. The parameters in these curves are identical except for $r^{\prime}$, which has the value 1.698 in one curve and 1.529 in the other. This is a $10 \%$ change in $r^{\prime}$. If we take the view that one of these model curves is an attempt to fit the other, we see by inspection of Fig. 5.3 that the fit is considerably worse than the fit in Fig. 5.2. We estimate that even with only a $5 \%$ change in $r^{\prime}$, the "fit" in Fig. 5.3 would be qualitatively worse than in Fig. 5.2. We therefore estimate that for $r^{\prime}$ values in the neighborhood of 1.7 (the region of most interest for us), the fitting procedure determines $r^{\prime}$ to within $5 \%$ of its true value.

Because of the tediousness of making each fit, the fitting algorithm was applied to only 39 signals. Based on these, we obtain the results for $\nu_{Q}, \sigma$, and $\eta$ shown in table 5.1. Casual inspection of the 39 data points 


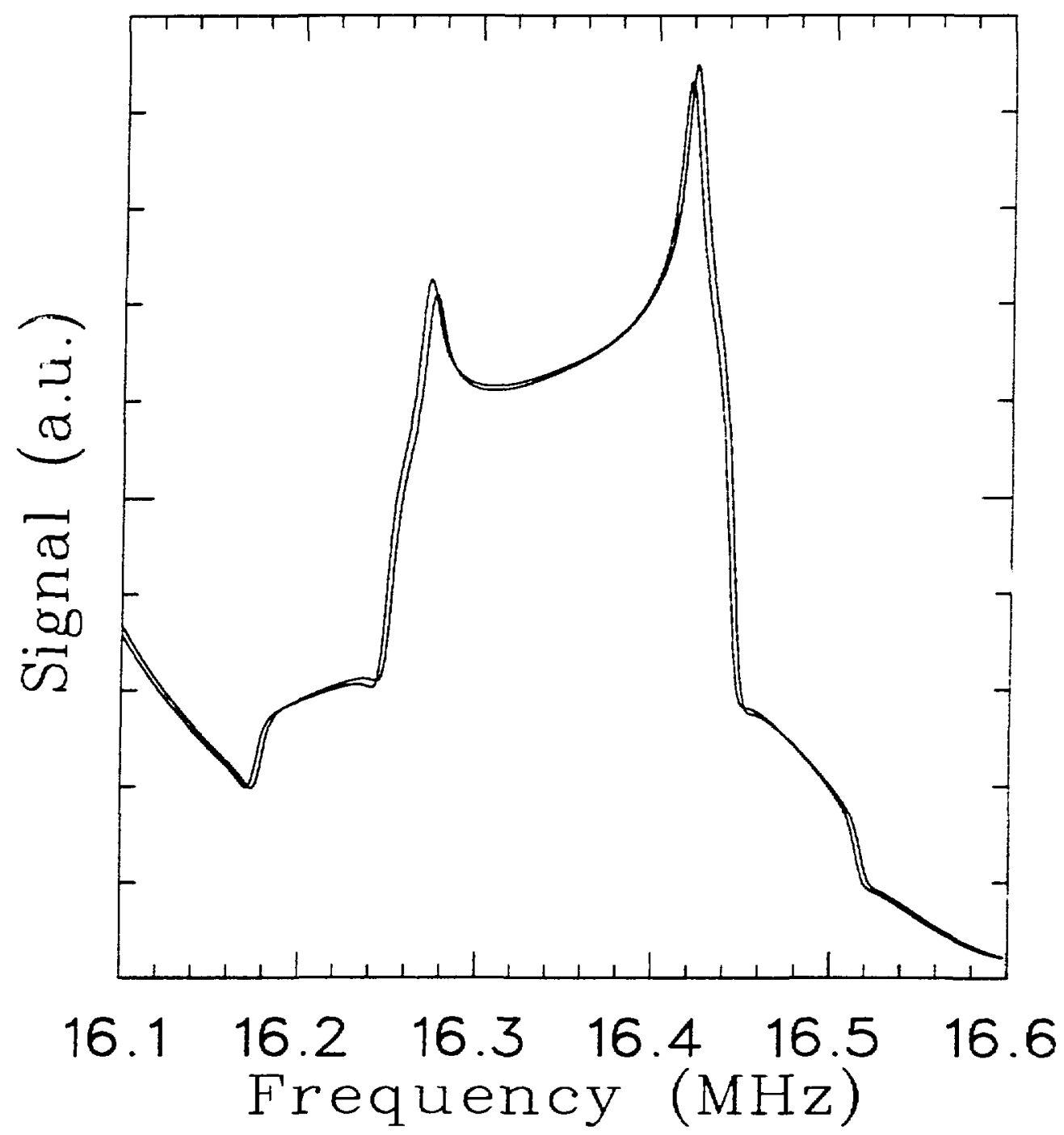

Figure 5.3: Two model curves are shown which differ only in that $r^{\prime}=1.698$ in one and $r^{\prime}=1.529$ in the other. From this we estimate tow well the fitting procedure determines $r^{\prime}$. (See the text). 
Table 5.1: Material parameters for solid $\mathrm{ND}_{3}$.

$\begin{array}{cccc} & \text { mean } & \text { standard dev. } & \text { stan. dev. of mean } \\ \nu_{Q} & 335.6 \mathrm{KHz} & 0.57 \% & 0.09 \% \\ \sigma & 0.0194 & 13.3 \% & 2.1 \% \\ \eta & 0.1263 & 4.5 \% & 0.72 \%\end{array}$

shows no significant correlations among the $\nu_{Q}, \sigma$, and $\eta$ values, and no significant correlations between these quantities and the magnitude or sign of the polarization.

To determine the vector polarization, $P$, we employ the thermal equilibrium method discussed in the previous chapter. Fig. 5.4 shows typical thermal data at $1.016 \mathrm{~K}$ and $25.00 \mathrm{KG}$ where $P=0.000515$. Unlike other signals shown in this chapter, the signal in Fig. 5.4 has the baseline signal subtracted. To make the signal visible, the vertical scale of Fig. 5.4 has been enlarged 500 times over that of Fig. 5.2. For simplicity, the circuit effects are here modeled with a third order polynomial which is determined from cubic regression on the wings of the signal. Summing the difference between the signal and this polynomial gives a good approximation to the constant $23 \alpha C$ given in (4.11).

By doing a similar summation on 147 distinct thermal signals, all collected at the same temperature and field, this constant was determined with a standard error of $0.44 \%$. With an uncertainty in the temperature measurement of $4 \%$ and an uncertainty in amplifier gain of $0.92 \%, P$ was determined using (4.11) with an uncertainty of $4.1 \%$. 


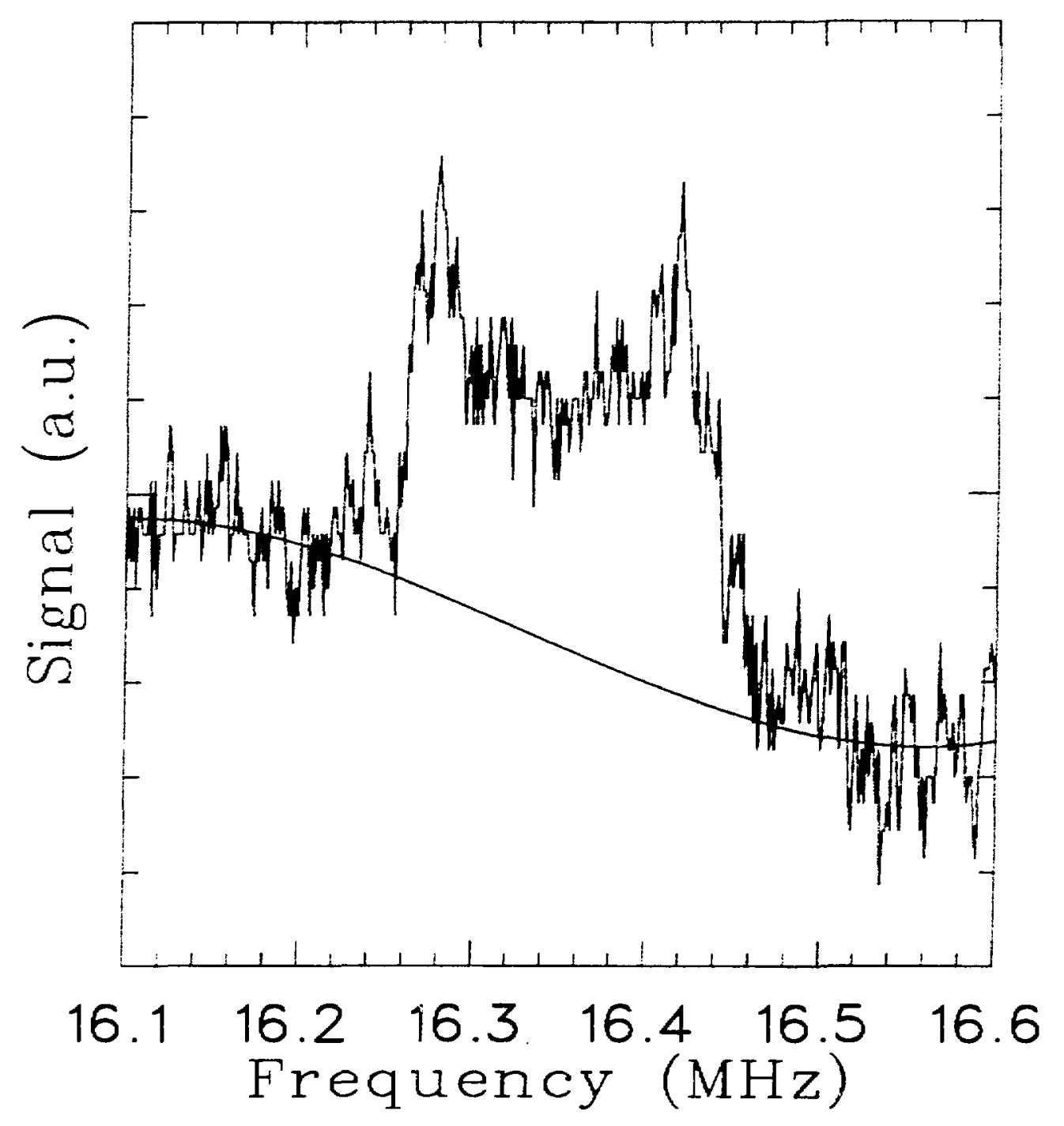

Figure 5.4: Typical $\wedge D_{3}$ thermal equilibrium signal, with baseline subtracted. Temperature and field values were $1.016 \mathrm{~K}$ and $25.00 \mathrm{KG}$ respectively. At thermal equiLibrium, this gives a vector polarization of 0.000515 . 
Using the $P$ values determined in this way, we use (4.13) and the corresponding $r^{\prime}$ value determined from fitting, to determine $A$. In Fig. 5.5 we plot the data on an $A$ vs $P$ plot similar to Fig. 2.2. The solid line represents the spin temperature curve. Horizontal error bars represent the uncertainty in $P$ discussed above. The vertical error bars represent only that part of the uncertainty in $A$ which derives from uncertainty in $P$ through (4.13). In other words, we have assumed that the uncertainty bars must be regarded as a lower bound on uncertainty in $A$. Nevertheless, for those data points with $|P|>0.3$ we see that the data is consistent with the spin temperature hypothesis. For E818, this data regime is of most importance since we always strove to operate at the largest available values of $|P|$. Those data points for which $|P|<0.3$ represent periods during which $|P|$ was rapidly changing, and the spin temperature hypothesis may be expected to fail. ${ }^{2}$

Having established equal spin temperature (EST) within experimental uncertainties for the region of interest to the $\mathrm{p}$-d scattering experiment, we chose to assume the validity of EST and apply the spin temperature method discussed in chapter 4. With this view, the spin tempcrature method and the thermal-equilibrium method give independent measurements of $P$. Fig. 5.6 shows a record of $P$ values measured during E818. Points indicate the average vector polarization for each run of E818. Averages were determined from the renormalized on-line data mentioned above.

\footnotetext{
${ }^{2}$ P-d scattering data collected during such periods was not used.
} 


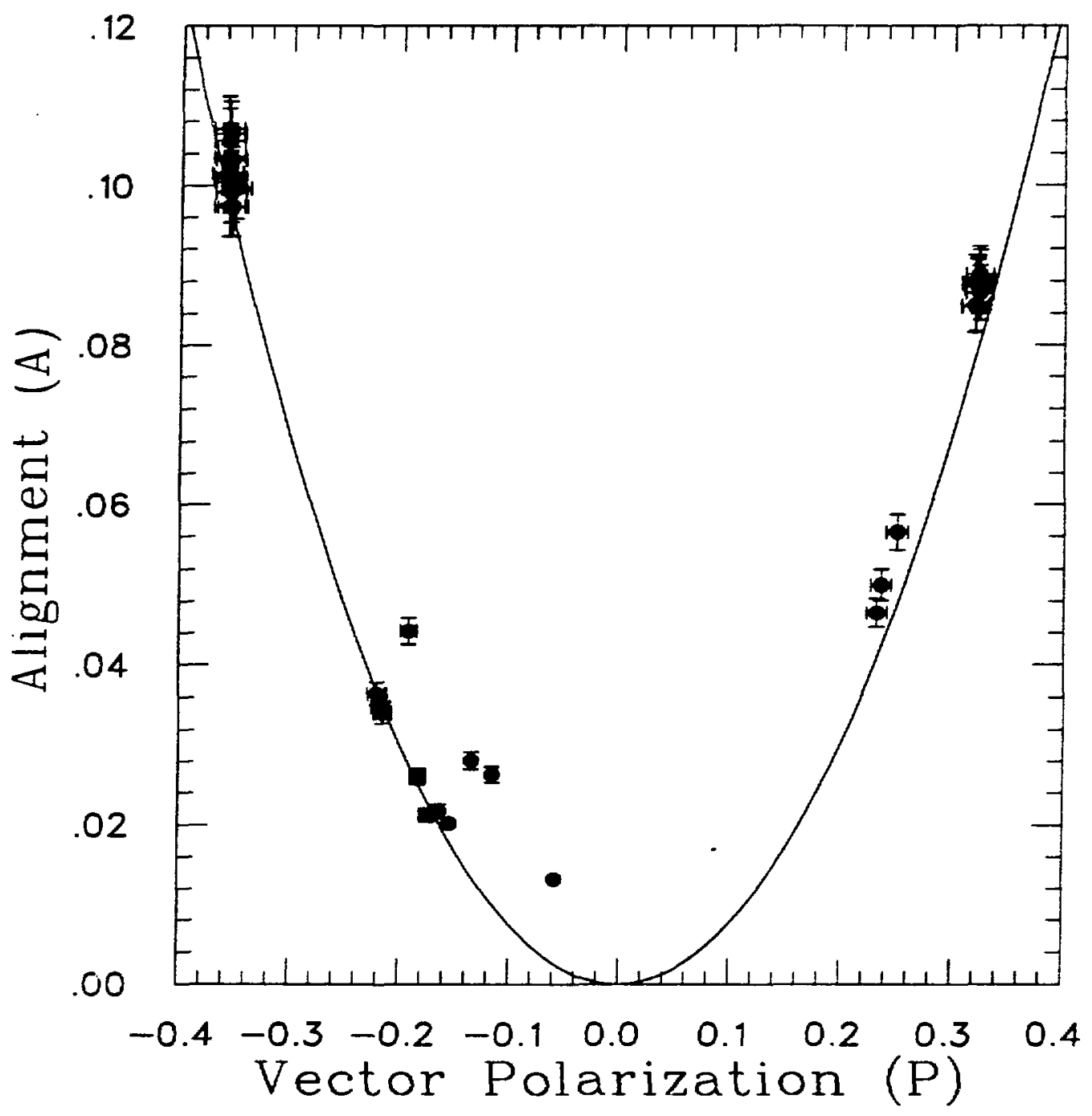

Figure 5.5: Data points for vector polarization and alignment. Points lying on the smooth curve satisfy the Spin Tempera:ure Theorem. 
To do this we separated the signals into those with $P>0$ and those with $P<0$ and examined the high-polarization signals for which both TE and curve fitting results were available. This showed

$$
P_{\text {fit }}=(1.093 \pm 0.011) \times P_{T E} \quad(P>0)
$$

and

$$
P_{f i t}=(1.038 \pm 0.027) \times P_{T E} \quad(P<0),
$$

where $P_{f i t}$ is the vector polarization determined by the spin temperature method, and $P_{T E}$ is the vector polarization determined with the TE method. The factor for $P>0$ was determined by averaging results from 10 signals, and the factor for $P<0$ was determined by averaging 15 signals. The uncertainties are standard deviations. With these factors we can determine an effective $P_{f i t}$ for each of the numerous renormalized on-line values. Since we regard $P_{f i t}$ and $P_{T E}$ as independent measurements of $P$, in Fig. 5.6 we report the average, $\left(P_{f i t}+P_{T E}\right) / 2$. We regard $\left(P_{f i i}-P_{T E}\right) / 2$ as the systematic error. The error bars in Fig. 5.6 are determined by adding this systematic error in quadrature with uncertainties in amplifier gain, temperature of TE measurements, and the standard error in integrals of the TE measurement. From day 12 to 23 , the accelerator beam was off and no data were collected. During this period, an incorrect capacitor in the low pass filter was discovered and replaced. For data collected prior to day 12, we used a numerical simulation to correct for this capacitor, before applying the analysis described above. 


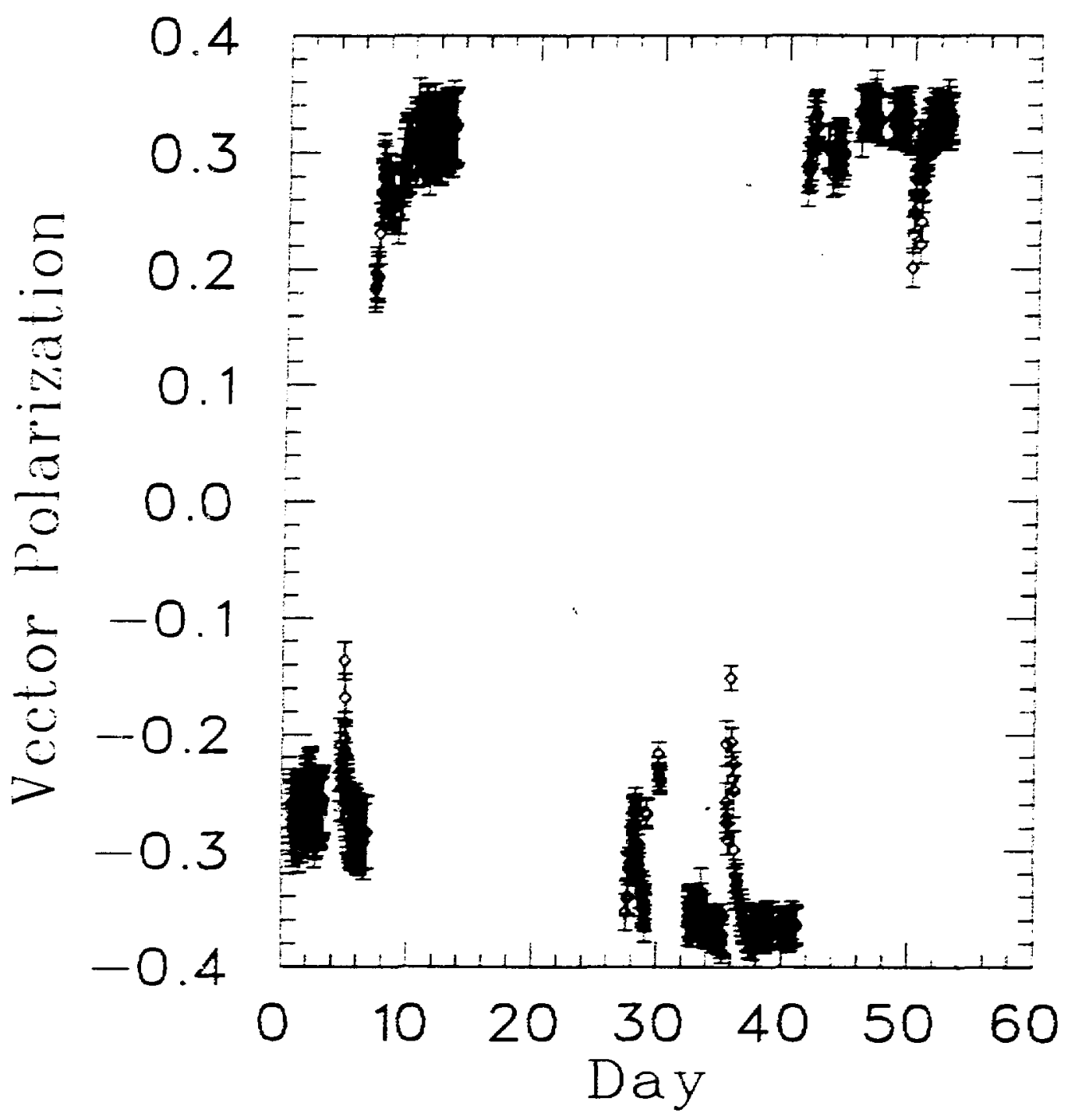

Figure 5.6: Vector polarization is time for Exlâ. 


\section{Appendix A}

\section{Kramers-Krönig Relation}

The Kramers-Krönig relation gives a general relationship between the real and imaginary parts of transfer functions associated with a wide class of linear systems. In this appendix we present the Kramers-Krönig relation in a form specifically tailored for our application. We begin with a general system and add restrictions until we obtain the desired equations. By "general system", we mean a mapping which takes real functions of time into real functions of time, i.e.,

$$
\mathcal{T}: \text { (real functions) } \longrightarrow \text { (real functions) }
$$

Thus if $s(t)$ is the stimulating function and $r(t)$ is the corresponding response. we have

$$
r=\mathcal{T}[s]
$$

Since $\mathcal{T}$ is a mapping from functions to functions, it is sometimes called a functional. Square brackets are used to indicate arguments of functionals. 
and smooth brackets to indicate arguments of functions. Explicitly writing the function arguments gives,

$$
r(t)=\mathcal{T}[s](t)
$$

The system of special interest to us is a time varying applied magnetic field and how it maps to the time varying magnetization which it induces. As we see below, the magnetic susceptibility $\chi$ is defined as the transfer function for this process.

It is notationally advantageous to define the generalized functions

$$
h_{z} \equiv \delta(t-z)
$$

and

$$
H(t, z) \equiv \mathcal{T}\left[h_{z}\right](t) .
$$

As a stimulus, $h_{z}$ causes a spike at time $z$, and $H(t, z)$ is the corresponding response.

\section{Linearity}

We assume $\mathcal{T}$ is linear, thus for a collection of stimulus functions $s_{j}(t)$, and time independent constants $a_{j}$, we have

$$
\mathcal{T}\left[\sum_{j} \alpha_{j} s_{j}\right]=\sum_{j} \alpha_{j} \mathcal{T}\left[s_{j}\right]=\sum_{j} \alpha_{j} r_{j} .
$$

We generalize this to a continuous sum (i.e., integral). So, for a $z$ parameterized set of stimulus functions,

$$
\mathcal{T}\left[\int \alpha(z) s_{z} d z\right]=\int \alpha(z) \mathcal{T}\left[s_{z}\right] d z=\int \alpha(z) r_{z} d z
$$


where we have used (A.1). We can expand any $s(t)$ in terms of $h_{z}$ with the identity

$$
s(t)=\int s(z) \delta(t-z) d z=\int s(z) h_{z}(t) d z .
$$

The only time dependence is now in $h_{z}(t)$. By linearity

$$
\begin{aligned}
r(t) & =\mathcal{T}[s](t)=\int s(z) \mathcal{T}\left[\dot{h}_{z}\right](t) d z \\
& =\int s(z) H(t, z) d z .
\end{aligned}
$$

\section{Time Translation Invariance}

Time translation invariance means that if we delay the stimulus, we delay the response by a corresponding amount. In other words, with $h_{z}$ as a stimulus we obtain

$$
\mathcal{T}\left[h_{z+c}\right](t)=\mathcal{T}\left[\tilde{h}_{z}\right](t-c) \text { for all } c,
$$

or from (A.2),

$$
H(t, z+c)=H(t-c, z) \text { for all } \mathrm{c} .
$$

Letting $z=0$ and changing notation so that $t=x$ and $c=y$, gives

$$
H(x, y)=H(x-y, 0) \text { for all } \mathrm{x} \text { and } \mathrm{y}
$$

By defining

$$
G(z) \equiv H(z, 0)
$$

$G$ may be interpreted as the response to a spike at time zero. We have from (A.3)

$$
r(t)=\int s(z) H(t, z) d z
$$




$$
\begin{aligned}
& =\int s(z) H(t-z, 0) d z \\
& =\int s(z) G(t-z) d z .
\end{aligned}
$$

\section{Causality}

Causality means that a spike at time $z$, such as $h_{z}$, must produce zero response for times prior to $z$. Thus

$$
0=\mathcal{T}\left[h_{z}\right](t)=H(t, z) \text { for } t<z
$$

which by definition of $G$ implies

$$
G(z)=0 \text { for } t<z .
$$

Note that all quantities defined so far are real.

\section{Fourier Transforms}

Using our definition of Fourier transform (see appendix B), we write

$$
\tilde{g}(\omega) \equiv \mathcal{F}[g](\omega) \equiv \int_{-\infty}^{\infty} g(t) e^{-i \omega t} d t .
$$

From the convolution theorem for Fourier transforms we have from (A.4)

$$
\tilde{r}(\omega)=\tilde{G}(\omega) \tilde{s}(\omega)
$$

$\tilde{g}(\omega)$ may be complex, but since $G(z)$ is a real function we have

$$
\tilde{G}(-\omega)=\int_{-\infty}^{\infty} G\left(\mathfrak{t}^{t}\right) e^{i \omega t} d t=\tilde{G}^{*}(\omega)
$$


Separating the real and imaginary parts we obtain

$$
\begin{aligned}
\operatorname{Re} \tilde{G}(-\omega) & =\operatorname{Re} \tilde{G}(\omega) \\
\operatorname{Im} \tilde{G}(-\omega) & =-\operatorname{Im} \tilde{G}(\omega) .
\end{aligned}
$$

These symmetries are important to us since they allow us to extend our knowledge of $\tilde{G}$ from the experimentally accessible positive frequency region to the inaccessible negative frequency region. $\tilde{G}(\omega)$ is sometimes called a transfer function.

We now analytically continue $\tilde{G}(\omega)$ into the complex plain. Letting $\omega=x+i y$, we have

$$
\begin{aligned}
\tilde{G}(\omega) & =\int_{-\infty}^{\infty} G(t) e^{-i \omega t} d t \\
& =\int_{-\infty}^{\infty} G(t) e^{-i x t} e^{y t} d t \\
& =\int_{0}^{\infty} e^{y t} \cos (x t) G(t) d t-i \int_{0}^{\infty} e^{y t} \sin (x t) G(t) d t \\
& \equiv u(x, y)+i v(x, y)
\end{aligned}
$$

where the lower integration limit has changed te zero in the penultimate step, since for causal $G, G(t)=0$ for $t$ negative. The last equality defines $u$ and $v$ when the integrals exist. Since $G$ is the response to a spike at time zero, we expect physically that $\mathrm{G}$ approaches zero for large $t$, or at least remains bounded. With this assumption, the integrals always exist for $y$ negative, and thus $u$ and $v$ are well defined in the lower half of the complex plain.

With the weak assumptions needed to interchange integration and partial differentiation [WW], it is straight forward to check that the CauchyReimann equations hold for $u$ and $v$. This shows that $\tilde{G}(\omega)$ is analytic in the 


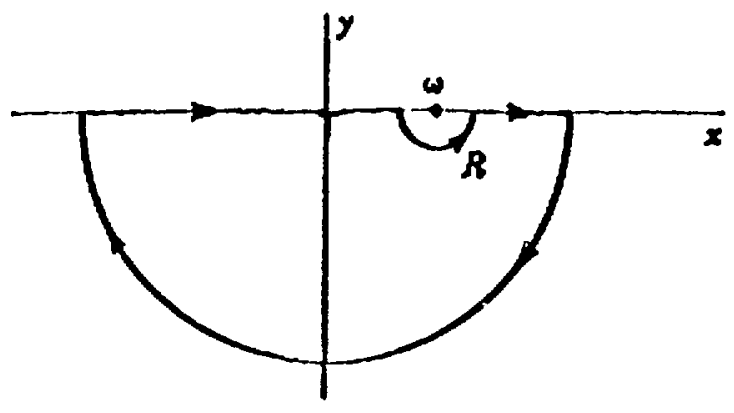

Figure A.1: Contour path for residue theorem.

lower half plane. We apply the Cauchy Integral Theorem with the contour shown in Fig. A.1. We assume that the integral over the large semi-circle goes to zero for large radius, though the physical meaning of this assumption seems somewhat unclear. Since $\omega$ is outside the contour, we may write

$$
\begin{aligned}
0 & =\frac{1}{2 \pi i} \oint \frac{\tilde{G}(z)}{z-\omega} d z \\
& =\frac{1}{2 \pi i}\left\{\int_{-\infty}^{\omega-R} \frac{\tilde{G}(x) d x}{x-\omega}+\int_{\omega+R}^{\infty} \frac{\tilde{G}(x) d x}{x-\omega}+\int_{\pi}^{2 \pi} \frac{\tilde{G}\left(\omega+R e^{i \theta}\right) i R e^{i \theta} d \theta}{R e^{i \theta}}\right\} \\
& =\frac{1}{2 \pi i}\left\{\mathcal{P} \int_{-\infty}^{\infty} \frac{\tilde{G}(x) d x}{x-\omega}+\tilde{G} i \pi\right\},
\end{aligned}
$$

where we have let $R \rightarrow 0$ for the last step. $\mathcal{P}$ indicates the principle part. Thus

$$
\tilde{G}(\omega)=\frac{-1}{\pi i} \mathcal{P} \int_{-\infty}^{\infty} \frac{\tilde{G}(x) d x}{x-\omega}
$$




$$
=\frac{-1}{\pi i}\left\{\mathcal{P} \int_{-\infty}^{\infty} \frac{\operatorname{Re} \tilde{G}(x) d x}{x-\omega}+i \mathcal{P} \int_{-\infty}^{\infty} \frac{\operatorname{Im} \tilde{G}(x) d x}{x-\omega}\right\}
$$

For real $\omega$, we take real and imaginary parts to obtain

$$
\begin{aligned}
\operatorname{Re} \tilde{G}(\omega) & =\frac{-1}{\pi} \mathcal{P} \int_{-\infty}^{\infty} \frac{\left.\operatorname{Im} \tilde{G}^{\prime} x\right)}{x-\omega} d x \quad(\omega \text { real }) \\
\operatorname{Im} \tilde{G}(\omega) & =\frac{1}{\pi} \mathcal{P} \int_{-\infty}^{\infty} \frac{\operatorname{Re} \tilde{G}(x)}{x-\omega} d x \quad(\omega \text { real })
\end{aligned}
$$

This is the Kramers-Krönig Relation.

\section{Susceptibility}

Using the conventional minus between real and imaginary parts of $\chi$, the susceptibility is defined by the equation

$$
M(\omega)=\chi(\omega) H(\omega)=\left(\chi^{\prime}(\omega)-i \chi^{\prime \prime}(\omega)\right) H(\omega)
$$

where $M$ is magnetization and plays the role of $\tilde{r}, H$ is the applied magnetic field and plays the role of $\tilde{s}$. (Note $H(\omega)$ and $H(t, z)$ are regarded as independent symbols in this appendix). $\chi(\omega)$ corresponds to $\tilde{G}$. Thus $\chi^{\prime}-i \chi^{\prime \prime}=\operatorname{Re} \tilde{G}+i \operatorname{Im} \tilde{G}$. From (A.8) we obtain

$$
\begin{aligned}
\chi^{\prime}(\omega) & =\frac{-1}{\pi} \mathcal{P} \int_{-\infty}^{\infty} \frac{\chi^{\prime \prime}(x)}{\omega-x} d x \\
\chi^{\prime \prime}(\omega) & =\frac{1}{\pi} \mathcal{P} \int_{-\infty}^{\infty} \frac{\chi^{\prime}(x)}{\omega-x} d x
\end{aligned}
$$

Note that the order of terms in the denominator has been reversed over that in (A.8). Since we must integrate over regions of negative frequency which 
are not accessible to measurement, we apply (A.7) to express the negative frequency part in terms of the positive frequency part. We obtain

$$
\begin{aligned}
\chi^{\prime}(-\omega) & =\chi^{\prime}(\omega) \\
\chi^{\prime \prime}(-\omega) & =-\chi^{\prime \prime}(\omega)
\end{aligned}
$$

With (A.9) and (A.10), a measurement in the positive frequency domain of only one of $\chi^{\prime}$ or $\chi^{\prime \prime}$ is sufficient to uniquely determine the other.

With the symmetries of (A.10), it is simple to show that (A.9) may be writien

$$
\begin{aligned}
\chi^{\prime}(\omega) & =\frac{2}{\pi} \int_{0}^{\infty} \frac{\chi^{\prime \prime}(x) x}{x^{2}-\omega^{2}} d x \\
\chi^{\prime \prime}(\omega) & =\frac{-2 \omega}{\pi} \int_{0}^{\infty} \frac{\chi^{\prime}(x)}{x^{2}-\omega^{2}} d x
\end{aligned}
$$

although we prefer the form (A.9) because of its similarity to a convolution integral. We show in the main text that there are computational advantages to the convolution form. 


\section{Appendix B}

\section{Fourier Transform Conventions and Results}

In this appendix we use a consistent notation to list conventions and theorems relating to the Fourier transiorm.

The Fourier transform will be defined by

$$
\check{g}(\omega) \equiv \mathcal{F}[g](\omega) \equiv \int_{-\infty}^{\infty} g(t) e^{-i \omega t} d t
$$

The inverse Fourier transform is then

$$
g(t)=\mathcal{F}^{-1}[\tilde{g}](t)=\frac{1}{2 \pi} \int_{-\infty}^{\infty} \tilde{g}(\omega) e^{i \omega t} d \omega .
$$

Parseval's theorem is

$$
\int_{-\infty}^{\infty}|g(t)|^{2} d t=\frac{1}{2 \pi} \int_{-\infty}^{\infty}|\tilde{g}(\omega)|^{2} d \omega
$$


If $\kappa$ is a constairt, and $g$ is defined by $g(t) \equiv f(\kappa t)$ then,

$$
\tilde{g}(\omega)=\frac{1}{|\kappa|} \tilde{f}\left(\frac{\omega}{\kappa}\right)
$$

The convolution theorem takes the form

$$
\int_{-\infty}^{\infty} f(\tau) g(t-\tau) d \tau=\mathcal{F}^{-1}[\tilde{f} \tilde{g}](t)
$$

or inversely

$$
\int_{-\infty}^{\infty} \tilde{f}\left(\omega^{\prime}\right) \tilde{g}\left(\omega-\omega^{\prime}\right) d \omega^{\prime}=2 \pi \mathcal{F}\left[\mathcal{F}^{-1}[\tilde{f}] \mathcal{F}^{-1}[\tilde{g}]\right](\omega)
$$

\section{Pertinent Transform Pairs}

$$
\begin{aligned}
& \delta_{\sigma}(t)=\frac{1}{\sqrt{2 \pi \sigma^{2}}} e^{\left(\frac{-t^{2}}{2 \sigma^{2}}\right)} \longleftrightarrow \quad \tilde{\delta}_{\sigma}(\omega)=e^{\frac{-\sigma^{2} \omega^{2}}{2}} \quad \text { (Gaussian) } \\
& \frac{\sigma}{\pi} \frac{1}{t^{2}+\sigma^{2}} \quad \longleftrightarrow \quad e^{-|\omega| \sigma} \quad \text { (Lorentzian) } \\
& \frac{1}{t} \longleftrightarrow \begin{cases}-i \pi & (\omega>0) \\
+i \pi & (\omega<0)\end{cases} \\
& \left\{\begin{array}{cc}
\frac{t i}{2} & (t>0) \\
\frac{-i}{2} & (t<0)
\end{array} \longleftrightarrow \quad \frac{1}{\omega}\right.
\end{aligned}
$$




\section{Appendix C}

\section{$f_{\eta}:$ Derivation and Properties}

\section{Functional Form of $f_{\eta}$}

In this appendix we compute the probability density function $f_{\eta}$, which was defined in the main text, as the probability density function for the quantity $\lambda(\theta, \psi)$ for randomly oriented crystal domains. In chapter 3 we defined $\lambda$ by

$$
\lambda(\theta, \psi) \equiv \frac{1}{2}\left(3 \cos ^{2} \theta-1-\eta \sin ^{2} \theta \cos (2 \psi)\right)
$$

and also showed that "random orientation" means

$$
\rho(\theta, \psi)=\frac{1}{4 \pi} \sin \theta
$$

Also from chapter 3 we know

$$
\begin{aligned}
& 0 \leq \theta \leq \pi \\
& 0 \leq \psi<2 \pi \\
& 0 \leq \eta \leq 1
\end{aligned}
$$


Rather than compute $f_{\eta}$ directly, we compute the distribution function associated with $f_{\eta}$, which we denote by ' $F$ '. Where as $f_{\eta}(z) d z$ may be interpreted as the fraction of domains which satisfy $z \leq \lambda(\theta, \psi) \leq z+d z$, $F(z)$ may be interpreted as the fraction of domains which satisfy $\lambda(\theta, \psi) \leq z$. $F$ and $f_{\eta}$ are related by

$$
f_{n}(z)=F^{\prime}(z) \equiv \frac{d F(z)}{d z} .
$$

To find $F(z)$, we must integrate $\rho(\theta, \psi)$ over regions of the $\theta-\psi$ plane which satisfy $\lambda(\theta, w) \leq z$.

It is convenient to shift the coordinate origin with the substitution

$$
\begin{aligned}
& \theta=t+\frac{\pi}{2} \\
& \psi=s+\frac{\pi}{2} .
\end{aligned}
$$

Then

$$
\begin{gathered}
\frac{-\pi}{2} \leq t \leq \frac{\pi}{2} \\
\frac{-\pi}{2} \leq s \leq \frac{3 \pi}{2} \\
\lambda(t, s)=\frac{1}{2}\left(3 \sin ^{2} t-1-\eta \cos ^{2} t \cos (2 s)\right)
\end{gathered}
$$

and

$$
\rho(t, s)=\frac{1}{4 \pi} \cos t
$$

The partial derivatives of $\lambda$ are given by

$$
\begin{aligned}
& \partial_{t} \lambda(t, s)=\frac{1}{2} \sin (2 t)[3+\eta \cos (2 s)] \\
& \partial_{s} \lambda(t, s)=\eta \cos ^{2} t \sin (2 s) .
\end{aligned}
$$


The extreme values of $\lambda$ must occur at the stationary points of $\lambda$, which are defined by the requirement that $\partial_{t} \lambda=\partial_{s} \lambda=0$. A straight forward examination of (C.4) shows that stationary points occur everywhere where $t= \pm \frac{\pi}{2}$, and at the discreet points where $(t, s)$ takes the values $\left(0, \frac{-\pi}{2}\right),(0,0),\left(0, \frac{\pi}{2}\right)$, $(0, \pi)$, and $\left(0, \frac{3}{2} \pi\right)$. For $t= \pm \frac{\pi}{2}, \lambda$ has the value 1 , which is its largest value. For $(t, s)$ equal to $\left(0, \frac{-\pi}{2}\right),\left(0, \frac{\pi}{2}\right)$, and $\left(0, \frac{3}{2} \pi\right), \lambda$ has a saddle point and takes the value $\frac{-1+\eta}{2}$. For $(t, s)$ equal to $(0,0)$ and $(0, \pi), \lambda$ has a minimum and takes its smallest value, $\frac{-1-\eta}{2}$. This is shown graphically in Fig. C.1, where the values of $\lambda$ at stationary points are also indicated.

$$
\begin{gathered}
\text { Since } \frac{-1-\eta}{2} \leq \lambda(t, s) \leq 1, F(z)=0 \text { for } z<\frac{-1-\eta}{2}, \text { and for } z>1, \\
F(z)=\int_{-\pi / 2}^{\pi / 2} d t \int_{-\pi / 2}^{3 \pi / 2} d s \frac{\cos t}{4 \pi}=1 \quad(z>1)
\end{gathered}
$$

As $z$ increases above $\frac{-1-\eta}{2}$, the region over which we must integrate $\rho$ enlarges about the two solid dots. To visualize this, it is useful to examine Fig. 3.5. To compute $F(z)$ we must integrate $\rho(\theta, \psi)$ over those values of $\theta$ and $\psi$ which satisfy $\lambda(\theta, \psi) \leq z$. We may visualize both a plane parallel to the $\theta-\psi$ plane in Fig. 3.5, and the plane's intersection with the $\lambda$ surface. The projection of this intersection onto the $\theta-\psi$ plane gives the boundary of the region over which we must integrate $\rho(\theta, \psi)$. The shift of origin caused by changing to $t, s$ variables does not effect this result. For $z$-values less than $\frac{-1+\eta}{2}$, but greater than $\frac{-1-\eta}{2}$, the boundary forms closed ovals, as shown in Fig. C.2a. We refer to this as case $A$. When $z$ exceeds $\frac{-1+\eta}{2}$, but remains less than 1 , the boundaries fail to close as shown in Fig. C.2b. We refer to this as case B. 


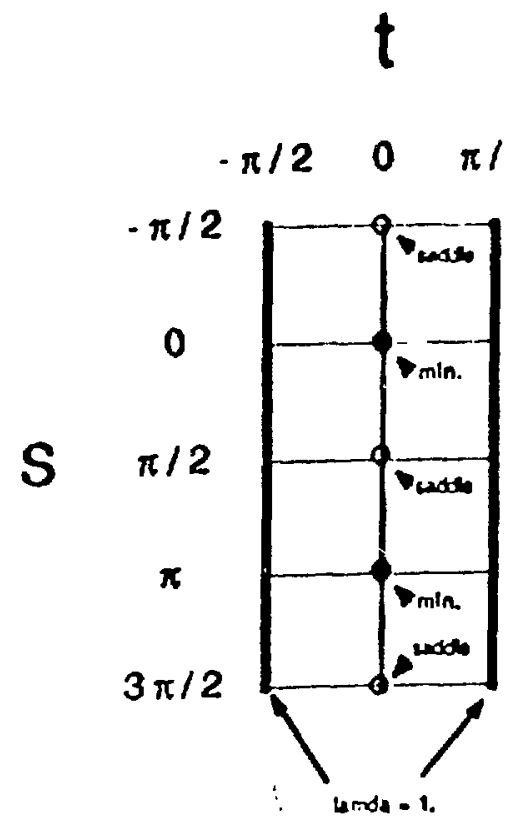

a) $\cdot \pi / 2 \quad 0 \quad \pi / 2$

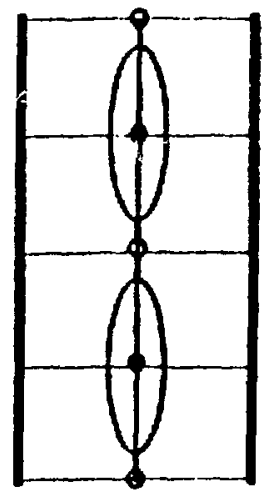

$\mathbf{S}$

b)

Figure C.1: a) The full range of the $t$ and $s$ variable is shown, with heavy lines and small circles marking the stationary points. $\lambda$ takes its maximum value of 1 everywhere on the heavy lines $\left(t= \pm \frac{\pi}{2}\right)$. It takes the value $\frac{-1+n}{2}$ at the circles marked "saddle," and it takes its minimum value of $\frac{-1-\pi}{2}$ at the points marked "min." b) A projected level curve for $\lambda=z>\frac{-1-\eta}{2}$. 
The integration may be simplified by noticing that $\lambda(t, s)$ and $\rho(t, s)$ are invariant under the three transformations

$$
\begin{gathered}
t \rightarrow t, \quad s \rightarrow s+\pi \\
t \rightarrow t, \quad s \rightarrow-s \\
t \rightarrow-t, \quad s \rightarrow s .
\end{gathered}
$$

With these symmetries, it is clear that we can write

$$
F(z)=8 \int_{\text {shaded region }} \int \frac{\cos t}{4 \pi} d t d s
$$

where "shaded region" is defined as the shaded region shown in Fig. C.2, and the factor 8 is included to account for the 7 regions which are related by the above invariances, to the shaded one.

To perform the integral in (C.2), we must express the "shaded region" analytically. For $\frac{-1-\eta}{2}<z<1$, we use

$$
z=\lambda(t, s)=\frac{1}{2}\left(3 \sin ^{2}(t)-1-\eta \cos ^{2}(t) \cos (2 s)\right)
$$

to give an implicit relationship between values of $t$ and $s$ which lie on the boundary of the integration region. Solving for $s$ gives

$$
s=\frac{1}{2} \arccos \left(\frac{3 \sin ^{2}(t)-1-2 z}{\eta \cos ^{2} t}\right) .
$$

This is valid for both case $\mathrm{A}$ and case B. For $t$ and $s$ inside the shaded region of Fig. C.2 we know that $t$ and $s$ are positive. The largest value of $t$ will occur when $s=0$. Setting $s=0$ in (C.6) and solving for $t$ we find

$$
t_{\max }=\arcsin \left(\sqrt{\left(\frac{2 z+1+\eta}{3+\eta}\right)}\right), \quad\left(\frac{-1-\eta}{2}<z<1\right) .
$$




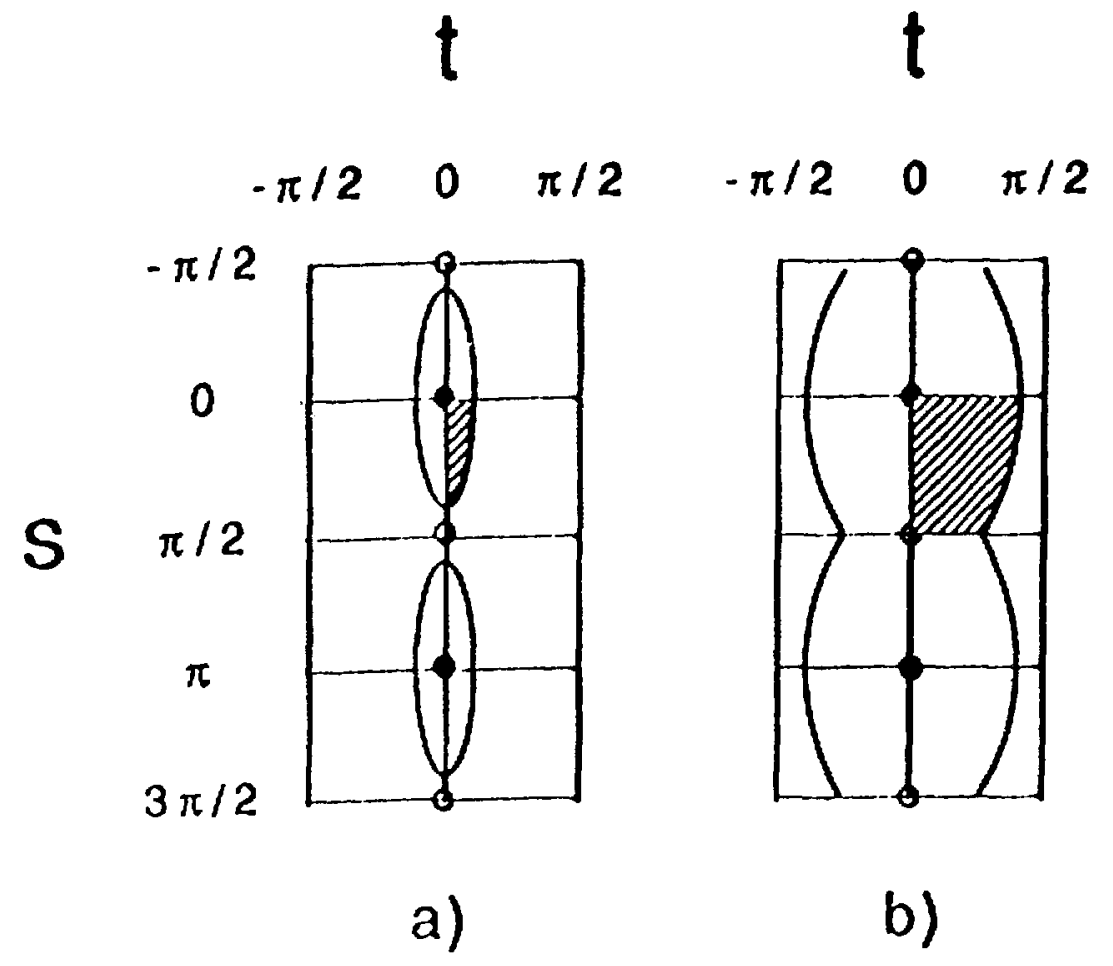

Figure C.2: Integration region. Because of symmetries in $\rho$ and $\lambda$, the interior of the projected level curves is equivalent to integrating $\rho(t, s)$ over the shaded region, and multiplying by 8 . a) $\frac{-1-\eta}{2} \leq z \leq \frac{-1+\eta}{2}$ b) $\frac{-1+\eta}{2}<z<1$. 
For case $\mathrm{A}, t$ ranges from 0 to $t_{\max }$, and the corresponding $s$ is given by (C.6). For case B, we define

$$
i_{\text {mid }}=\arcsin \left(\sqrt{\left(\frac{2 z+1-\eta}{3-n}\right)}\right) .
$$

This results from solving (C.6) when $s=\frac{\pi}{2}$ and corresponds to the value of $t$ at which the boundary breaks away from $s=\frac{\pi}{2}$ (see Fig. C.2). For case B, integration is broken into two parts, where $s=\frac{\pi}{2}$ for $0<t_{\text {mid }}$, and $s$ is given by (C.6) for $t_{\text {mid }}<t<t_{\max }$.

Combining these results, and writing $s_{z}(t)$ for the right hand side of (C.6) we have

$$
F(z)=\left\{\begin{array}{lc}
0 & z \leq \frac{-1-\eta}{2} \\
8 \int_{0}^{t_{\max }} d t \int_{0}^{s_{z}(t)} d s^{\prime} \frac{\cos t}{4 \pi} & \frac{-1-\eta}{2}<z<\frac{-1+\eta}{2} \\
8\left(\int_{0}^{t_{m+d}} d t \int_{0}^{\pi / 2} d s^{\prime} \frac{\cos t}{4 \pi}+\int_{t_{\operatorname{mid}}}^{t_{\max }} d t \int_{0}^{s_{z}(t)} d s^{\prime} \frac{\cos (t)}{4 \pi}\right) & \frac{-1+\eta}{2}<z<1 \\
& \text { (case A) } \\
1 & z \geq 1 .
\end{array}\right.
$$

Substituting the explicit forms for $s_{z}$, we can compute

$$
\begin{aligned}
8 \int_{t_{1}}^{t_{2}} d t \int_{0}^{s_{z}(t)} d s^{\prime} \frac{\cos t}{4 \pi} & =\frac{2}{\pi} \int_{t_{1}}^{t_{2}} \frac{1}{2} \arccos \left[\frac{3 \sin ^{2} t-1-2 z}{\eta \cos ^{2}(t)}\right] \cos t d t \\
& =\frac{1}{\pi} \int_{\sin \left(t_{1}\right)}^{\sin \left(t_{2}\right)} \arccos \left[\frac{3 u^{2}-1-2 z}{\eta\left(1-u^{2}\right)}\right] d u,
\end{aligned}
$$

where the last step follows from the substitution $u=\sin t$. We also note that

$$
8 \int_{0}^{t_{m i d}} d t \int_{0}^{\frac{\pi}{2}} d s^{\prime} \frac{\cos t}{4 \pi}=\frac{2}{\pi}\left(\frac{\pi}{2}-0\right)\left(\sin \left(t_{m i d}\right)-\sin (0)\right)
$$




$$
\begin{aligned}
& =\sin \left(t_{m i d}\right) \\
& =\sqrt{\frac{2 z+1-\eta}{3-\eta}} .
\end{aligned}
$$

Substituting the forms for $t_{m i d}$ and $t_{\max }$ we can write

$$
F(z)=\left\{\begin{array}{lc}
0 & z<\frac{-1-\eta}{2} \\
\frac{1}{\pi} \int_{0}^{\sqrt{\frac{z z+1+n}{3+\eta}}} \arccos \left[\frac{3 u^{2}-1-2 z}{\eta\left(1-u^{2}\right)}\right] d u & \frac{-1-n}{2}<z<\frac{-1+n}{2} \\
\sqrt{\frac{2 z+1-\eta}{3-\eta}}+\frac{1}{\pi} \int \frac{\sqrt{\frac{2 z+1+\eta}{3+\eta}}}{\sqrt{\frac{2 z+1-\eta}{3-\eta}}} \arccos \left[\frac{3 u^{2}-1-2 z}{\eta\left(1-u^{2}\right)}\right] d u & \frac{-1+\eta}{2}<z<1 \\
1 & \text { (case A) } \\
& \text { otherwise }
\end{array}\right.
$$

To find $f_{\eta}$, we must compute $F^{\prime}$. To this end, we recall a result from Calculus which says that for a function $f$ which can be expressed in terms of functions $g_{1}(x), g_{2}(x)$, and $h(x, y)$ by the formula

$$
f(z)=\int_{g_{1}(z)}^{g_{2}(z)} h(u, z) d u,
$$

the derivative of $f$ may be written

$$
f^{\prime}(z)=-h\left[g_{1}(z), z\right] g_{1}^{\prime}(z)+h\left[g_{2}(z), z\right] g_{2}^{\prime}(z)+\int_{g_{1}(z)}^{g_{2}(z)} \partial_{z} h(u, z) d u .
$$

Applying this to case $\mathrm{A}$, we let $g_{1}(z)=0, g_{2}(z)=\sqrt{\frac{2 z+1+\eta}{3+\eta}}$, and $h(u, z)=\arccos \left[\frac{3 u^{2}-1-2 z}{\eta\left(1-u^{2}\right)}\right]$. Then

$$
h\left[g_{2}(z), z\right]=\arccos \left[\frac{3 \frac{2 z+1+\eta}{3+\eta}-1-2 z}{\eta\left(1-\frac{2 z+1+\eta}{3+\eta}\right)}\right]
$$




$$
\begin{aligned}
& =\arccos \left[\frac{3(2 z+1+\eta)-(2 z+1)(3+\eta)}{\eta(3+\eta-2 z-1-\eta)}\right] \\
& =\arccos \left[\frac{2 \eta(1-z)}{2 \eta(1-z)}\right] \\
& =0 .
\end{aligned}
$$

Also

$$
g_{1}^{\prime}(z)=0
$$

Equation (C'.8) then gives

$$
f_{\eta}=F^{\prime}(z)=\frac{1}{\pi} \int_{0}^{\sqrt{\frac{2 z+1+\eta}{3+\eta}}} \partial_{z} h(u, z) d u \quad(\text { case } \mathrm{A}) .
$$

For the integral in case B, we let $g_{1}(z)=\sqrt{\frac{2 z+1-\eta}{3-\eta}}$, with $g_{2}(z)$ and $h(u, z)$ the same as in case $\mathrm{A}$. For case $\mathrm{B}$, we must also differentiate the first term in $(C . \bar{T})$ case B. Since as before, $h\left(\sqrt{\frac{2 z+1+n}{3+\eta}}, z\right)=0$, and

$$
\begin{aligned}
h\left(\sqrt{\frac{2 z+1-\eta}{3-\eta}}, z\right) & =\arccos \left[\frac{3 \frac{2 z+1-\eta}{3-\eta}-1-2 z}{\eta\left(1-\frac{2 z+1-\eta}{3-\eta}\right)}\right] \\
& =\arccos [-1] \\
& =\pi,
\end{aligned}
$$

we obtain

$$
\begin{aligned}
f_{\eta}= & \frac{2}{2 \sqrt{(3-\eta)(2 z+1-\eta)}}+ \\
& \frac{1}{\pi}\left\{\frac{-2 \pi}{\sqrt{(3-\eta)(2 z+1-\eta)}}+\int \frac{\sqrt{\frac{2 z+1+\eta}{3+\eta}}}{\sqrt{\frac{2 z+1-\eta}{3-\eta}}} \partial_{z} h(u, z) d u\right\} \\
= & \frac{1}{\pi} \int \frac{\sqrt{\frac{2 z+1+\eta}{3+\eta}}}{\sqrt{\frac{2 z+1-\eta}{3-\eta}}} \partial_{z} h(u, z) d u \quad \text { (case B). }
\end{aligned}
$$


For $z<\frac{-1-\eta}{2}$ and $z>1, F(z)$ is constant, so $F^{\prime}(z)=0$ in those regions. Thus

$$
f_{\eta}=\left\{\begin{array}{lcc}
\frac{1}{\pi} \int_{0}^{\sqrt{\frac{2 z+1+\eta}{3+\eta}}} \partial_{z} h(u, z) d u & \frac{-1-\eta}{2} \leq z \leq \frac{-1-\eta}{2} & \text { (case A) } \\
\frac{1}{\pi} \int \frac{\sqrt{\frac{2 z+1+\eta}{3+\eta}}}{\sqrt{\frac{2 z+1-\eta}{3-\eta}}} \partial_{z} h(u, z) d u & \frac{-1+\eta}{2}<z \leq 1 & \text { (case B) } \\
0 & \text { otherwise }
\end{array},\right.
$$

where $h(u, z)=\arccos \left[\frac{3 u^{2}-1-2 z}{n\left(1-u^{2}\right)}\right]$. Examination of the integration limits shows that $f_{\eta}$ is continuous at the boundary between the separate domains on which it is defined. (For the boundary between case $A$ and case $B$, continuity means $f_{n} \rightarrow+\infty$ from both sides).

To compute the integrals in (C.9), we evaluate $\partial_{z} h(u, z)$ explicitly. Straight forward differentiation gives

$$
\begin{aligned}
\partial_{z} h(u, z) & =\frac{2}{\sqrt{\left[\eta-1-2+(3-\eta) u^{2}\right]\left[\eta+1+2 z-(3+\eta) u^{2}\right]}} \\
& =\frac{2}{\sqrt{3-\eta} \sqrt{3+\eta}} \frac{1}{\sqrt{\left[\frac{\eta-1-2 z}{3-\eta}+u^{2}\right]\left[\frac{\eta+1+2 z}{3+\eta}-u^{2}\right]}} .
\end{aligned}
$$

The integrals over $u$ in (C.9) can be evaluated in terms of elliptic integrals.

For case $A$ we make the substitutions

$$
\begin{aligned}
& a=\sqrt{\frac{\eta-1-2 z}{3-\eta}} \\
& b=\sqrt{\frac{\eta+1+2 z}{3+\eta}} .
\end{aligned}
$$

From (C.9) we obtain

$$
f_{n}(z)=\frac{2}{\pi} \int_{0}^{b} \frac{d u}{\sqrt{\left[a+u^{2}\right]\left[b-u^{2}\right]}}(\text { case } \mathrm{A})
$$




$$
=\frac{1}{\pi} \frac{1}{\sqrt{\eta(1-z)}} K\left(\frac{(3-\eta)(\eta+1+2 z)}{4 \eta(1-z)}\right) \quad(\text { case } A),
$$

where the last equality follows from Abramowitz and Stegun [AS] Equation 17.5.51. $I$ is the complete elliptic function defined by

$$
K(x)=\int_{0}^{\pi / 2} \frac{1}{\sqrt{1-x \sin ^{2} \theta}} d \theta .
$$

For case $B$ we make the substitutions

$$
\begin{aligned}
& a=\sqrt{\frac{2 z+1+\eta}{3+\eta}} \\
& b=\sqrt{\frac{2 z+1-\eta}{3-\eta}},
\end{aligned}
$$

and from (C.9) we obtain

$$
\begin{aligned}
f_{\eta}(z) & =\frac{2}{\pi} \frac{1}{\sqrt{3-\eta} \sqrt{3+\eta}} \int_{b}^{\pi} \frac{d u}{\sqrt{\left[-b+u^{2}\right]\left[a^{2}-u^{2}\right]}} \text { (case B) } \\
& =\frac{2}{\pi \sqrt{(3-\eta)(\eta+1+2 z)}} \Gamma\left(\frac{4 \eta(1-z)}{(3-\eta)(\eta+1+2 z)}\right) \quad \text { (case B), }
\end{aligned}
$$

where the last inequality follows from Abramowitz and Stegun [AS] equation 17.4.44.

Combining all cases we have

$$
f_{\eta}(z)= \begin{cases}\frac{1}{\pi \sqrt{\eta(1-z)}} K\left(\frac{(3-\eta)(\eta+1+2 z)}{4 \eta(1-z)}\right) & \text { for } \frac{-1-\eta}{2} \leq z \leq \frac{-1+\eta}{2} \\ \frac{2}{\pi \sqrt{(3-\eta) \eta+1+2 z)}} K\left(\frac{4 \eta(1-z)}{(3-\eta)(\eta+1+2 z)}\right) & \text { for } \frac{-1+\eta}{2} \leq z \leq 1 \\ 0 & \text { otherwise. }\end{cases}
$$

This is the formula for $f_{\eta}$ used in chapter 3 . 
The function $K$ takes finite values everywhere except at 1 , where it takes the value $+\infty$. Thus $f_{\eta}$ is finite, except at $z=\frac{-1+\eta}{2}$. We also note that

$$
\int_{-\infty}^{\infty} f_{n}(z) d z=\int_{-\infty}^{\infty} F^{\prime}(z) d z=F(+\infty)-F(-\infty)=1
$$

\section{The Hamada form}

An alternative form of the function $g^{+}$which we described in chapter 3 , has been used in the literature [Ha] $[\mathrm{Sp}]$. Although we have not used this form in our work, in this section we show the relationship between $f_{\eta}$, and this alternative form, which we denote with ' $G$ '. In chapter 3 , equation (3.20) we wrote

$$
g^{+}(x)=\frac{2}{\nu_{Q}} \int_{-\infty}^{\infty} f_{\eta}\left(\frac{2 u}{\nu_{Q}}\right) \delta_{\sigma}(x-u) d u .
$$

To conform with the above mentioned literature, we rescale our frequency units so that $\frac{2}{\nu_{Q}}=1 \mathrm{~Hz}$. $G$ is then defined by

$$
G(x) \equiv \int_{-\infty}^{\infty} f_{\eta}(z) \delta_{\sigma}(x-z) d z
$$

$\delta_{\sigma}$ denotes a general normalized function of width $\sigma$, for example a Lorentzian or Gaussian. We also define

$$
\begin{aligned}
& p \equiv \sqrt{2 \eta}, \\
& q \equiv \sqrt{3-\eta}, \\
& r \equiv \sqrt{3+\eta} .
\end{aligned}
$$


Breaking the integral at the natural domain boundaries of $f_{\eta}$, we write

$$
\begin{aligned}
G(x)= & \int_{\frac{-1-\eta}{2}}^{1} f_{\eta}(z) \delta_{\sigma}(x-z) d z \\
= & \int_{\frac{-1-\eta}{2}}^{\frac{-1+\eta}{2}} f_{\eta}(z) \delta_{\sigma}(x-z) d z+\int_{\frac{-1+\eta}{2}}^{1} f_{\eta}(z) \delta_{\sigma}(x-z) d z \\
= & \frac{2}{\pi p} \int_{\frac{-1-\eta}{2}}^{\frac{-1+\eta}{2}} \delta_{\sigma}(x-z) \frac{1}{\sqrt{2-2 z}} K^{-}\left(\frac{q^{2}(\eta+1+2 z)}{p^{2}(2-2 z)}\right) d z \\
& +\frac{2}{\pi q} \int_{\frac{-1+\eta}{2}}^{1} \delta_{\sigma}(x-z) \frac{1}{\sqrt{\eta+1+2 z}} \Gamma\left(\frac{p^{2}(2-2 z)}{q^{2}(\eta+1+2 z)}\right) d z .
\end{aligned}
$$

Define $I_{1}$ and $I_{2}$ respectively, as the first and second terms in the last equation.

For $I_{1}$ we make the change of variable $t=\sqrt{2-2 z}$, so $d t=\frac{-d z}{\sqrt{2-2 z}}$ and $z=1-\frac{t^{2}}{2}$. Then

$$
\begin{aligned}
I_{1} & =\frac{2}{p \pi} \int_{\sqrt{3+\eta+1}}^{\sqrt{3-\eta+1}} \delta_{\sigma}\left(x-\left[1-\frac{t^{2}}{2}\right]\right) K\left(\frac{q^{2}\left(\eta+1+2-t^{2}\right)}{p^{2}\left(2-\left(\left[2-t^{2}\right]\right)\right.}\right)(-d z) \\
& =\frac{2}{p \pi} \int_{q}^{\tau} \delta_{\sigma}\left(x-\psi_{1}(t)\right) K\left(\frac{q^{2}\left(r^{2}-t^{2}\right)}{p^{2} t^{2}}\right) \\
& =\frac{2}{p \pi} \int_{q}^{r} \delta_{\sigma}\left(x-\psi_{1}(t)\right) K\left(k_{1}^{2}(t)\right)
\end{aligned}
$$

where we have defined $\psi_{1}(t) \equiv 1-\frac{t^{2}}{2}$ and $k_{1}(t)=\frac{q}{p}\left(\frac{r^{2}}{t^{2}}-1\right)$.

For $I_{2}$ we make the substitution $t=\sqrt{\eta+1+2 z}$ so $z=\frac{t^{2}-\eta-1}{2}$ and $d t=\frac{d z}{\sqrt{\eta+1+2 z}}$. This gives

$$
\begin{aligned}
I_{2} & =\frac{2}{q \pi} \int_{\sqrt{2 \eta}}^{\sqrt{\eta+3}} \delta_{\sigma}\left(x-\psi_{2}(t)\right) K^{-}\left(\frac{p^{2}\left(2-t^{2}+\eta+1\right)}{q^{2}\left(\eta+1+z^{2}-\eta-1\right)}\right) d t \\
& =\frac{2}{q \pi} \int_{p}^{r} \delta_{\sigma}\left(x-\psi_{2}(t)\right) K_{2}\left(k_{2}^{2}(t)\right) d t
\end{aligned}
$$


where

$$
\begin{aligned}
\psi_{2}(t) & \equiv \frac{1}{2}\left(t^{2}-\eta-1\right) \\
k_{2}(t) & \equiv \frac{p}{q} \sqrt{\frac{r^{2}}{t^{2}}-1}
\end{aligned}
$$

We summarize the resuits with

$$
G(x)=\frac{2}{p \pi} \int_{\eta}^{r} \delta_{\sigma}\left(x-\psi_{1}(t)\right) H\left(k_{1}^{2}(t)\right) d t+\frac{2}{q \pi} \int_{p}^{r} \delta_{\sigma}\left(x-\psi_{2}(t)\right) H_{1}\left(k_{2}^{2}(t)\right) d t,
$$

where

$$
\begin{aligned}
p & \equiv \sqrt{2 \eta} \\
q & \equiv \sqrt{3-\eta}, \\
r & \equiv \sqrt{3+\eta}, \\
\xi_{1}(t) & \equiv 1-\frac{1}{2} t^{2}, \\
\psi_{2}(t) & \equiv \frac{1}{2}\left(t^{2}-\eta-1\right), \\
k_{1}(t) & \equiv \frac{q}{p} \sqrt{\frac{r^{2}}{t^{2}}-1} \text { and } \\
k_{2}(t) & \equiv \frac{p}{q} \sqrt{\frac{r^{2}}{t^{2}}-1} .
\end{aligned}
$$

Comparing this result with Hamada's [Ha] shows two differences.

One is an overall factor of 2 , which is not important since our application needs only the normalized shape of the function. The second difference is that the functions $k_{1}$ and $k_{2}$ are squared as arguments to $K$, where as Hamada's are not. This results from Hamada's implicit definition of $K^{-}(x)$ which differs 
from the Abramowitz and Stegun [AS] convention which we have used.

$$
\begin{aligned}
K_{\text {Hamada }}(x) & =\int_{0}^{\frac{\pi}{2}} \frac{d \theta}{\sqrt{1-x^{2} \sin \theta}} \\
K_{\text {A.S. }}(x) & =\int_{0}^{\frac{\pi}{2}} \frac{d \theta}{\sqrt{1-x \sin \theta}} .
\end{aligned}
$$

Both conventions are used in the mathematical literature [JE]. 


\section{Appendix D}

\section{Preparation of Target Material}

For the dynamic nuclear polarization process to be effective, it is necessary for the target material to be cooled to approximately $0.5 \mathrm{~K}$, and for paramagnetic centers to be introduced into the material [AG]. In our material, paramagnetic centers were created with an irradiation process described in this appendix. In order to remove heat generated by microwave stimulation and by beam heating, it is necessary for the target material to be broken into pieces ranging in size from 1.5 to $3.0 \mathrm{~mm}$. This allows the cooling fluid to flow between the pieces and efficiently remove heat from the solid. In this appendix, we also describe the method by which gasecus $\mathrm{ND}_{3}$ was frozen and broken into pieces of the required size.

\section{Freezing Gaseous $\mathrm{ND}_{3}$}

Fig. D.1 shows a schematic representation of the apparatus used to freeze 


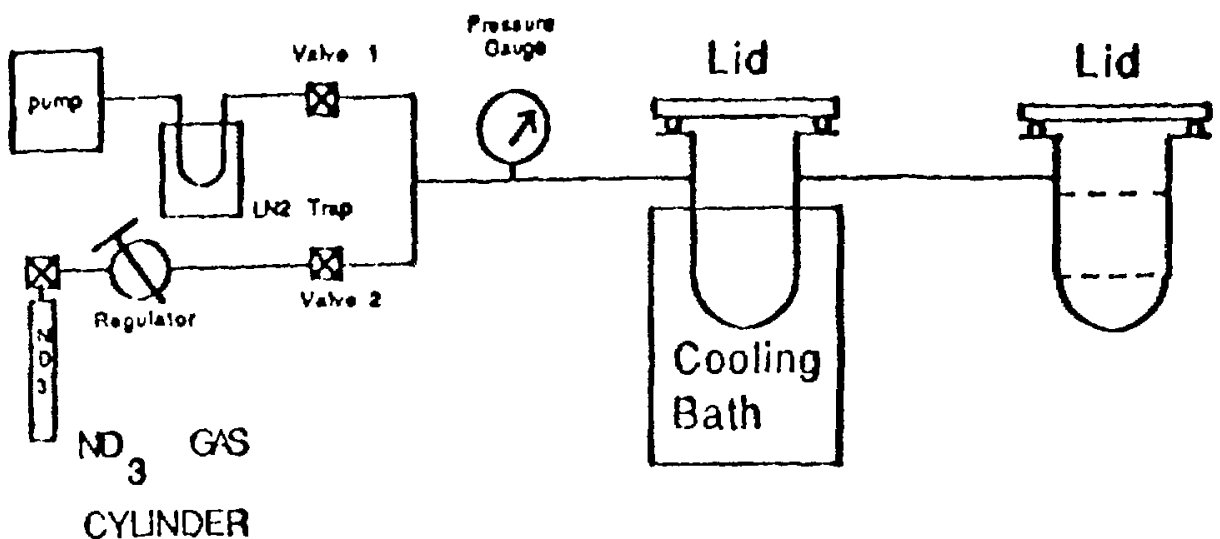

GAS

MANIFOLD
Freezing Chamber

(fc)
Sifling

Chamber

(sc)

Figure D.1: Schematic representation of $\mathrm{ND}_{3}$ freezing apparatus. 
the gaseous $N D_{3}$. Two cylindrical glass chambers each about $16 \mathrm{~cm}$ high and $8 \mathrm{~cm}$ in diameter were constructed and fitted with lids made from plexi-glass with a groove cut to hold an o-ring which sealed against the flat 1upper lips of the glass chambers (sce Fig. D.1). Glass tubing was attached to these chambers in such a fashion that vacuum hoses could be clamped onto them to form vacuum tiglit connections. As indicated in Fig. D.1, the chanter designated the freezing chamber ( $\mathrm{fc}$ ) has two such tubes, and the chamber designated the sifting chamber (sc) has only one. The sc contains a stainless steel sieve whose upper partion consists of a stainless steel plate with many $3 \mathrm{~mm}$ holes in it. Located $4 \mathrm{~cm}$ below this is a stainless steel screen which passes particles smaller than $1.5 \mathrm{~mm}$.

In operation, the vacuum pump is used to remove atmospheric gases from the system. The pump we used reduced the pressure to approximately 200 Torr. After isolating the pump from the system (by closing valve 1), the freezing chamber is immersed in a cooling bath of liquid nitrogen ( $\left.\mathrm{LN}_{2}\right)$, and $\mathrm{ND}_{3}$ is introduced and allowed to solidify in the fc.

The cooling bath was originally conceived as a mixture of ethyl alcohol and powdered dry ice, nixed and placed in a Dewar. The temperature of this combination is about $190 \mathrm{~K}$, and since $\mathrm{ND}_{3}$ contenses at $242 \mathrm{~K}$ we expected $\mathrm{ND}_{3}$ to condense in the fc. Condensation did not occur however and we were forced to use a cooling bath of $\mathrm{LN}_{2}$ which has a temperature of $77 \mathrm{~K}$. The disadvantage of using $\mathrm{LN}_{2}$ is that more atmospheric gases will condense along with $\mathrm{ND}_{3}$, at the colder $\mathrm{LN}_{2}$ temperature. 
For maximal heat conduction within the target material, it is desirable to have the large crystal domains which result form slow freezing. For this reason, we removed the $\mathrm{LN}_{2}$ bath and allowed the fc to warm until all the $\mathrm{ND}_{3}$ had melted. We then immersed the fc in the ethanol-dry ice mixture $(190 \mathrm{~K})$ and allowed it to refreeze slowly. $\left(\mathrm{ND}_{3}\right.$ freezes at $\left.199 \mathrm{~K}\right)$. This refreezing took about one hour, and produced a nearly transparent $\mathrm{ND}_{3}$ ice.

At this point, the chambers were brought to atmospheric pressure, the lids remove, and both $\mathrm{fc}$ and sc filled with $\mathrm{LN}_{2}$. The rapid temperature change in the sc formed cracks in the solid $N D_{3}$. With a stainless steel rod the immersed $\mathrm{ND}_{3}$ ice was broken into large chunks. Using a stainless steel spoon, these chunks were transferred one at a time to the sc, where they were ground against the upper sieve until they broke into pieces which could pass through the upper sieve. Pieces which were too small, passed through the lower sieve to the bottom of the sc. As the space between sieves became too full of $\mathrm{ND}_{3}$, this material was transferred to a storage bottle kept under $\mathrm{LN}_{2}$.

We had originally planned to recover the $\mathrm{ND}_{3}$ which had passed to the bottom of the sc by closing the system, pumping out the $\mathrm{N}_{2}$ while keeping both sc and fe cold, and finally allowing the sc to warm while still maintaining the fc at a cold temperature. The $\mathrm{ND}_{3}$ in the sc would raporize and recondense in the fc. It would then be possible to add a new bottle of $N D_{3}$ to the recycled $N D_{3}$, and repeat the whole procedure. The problem with this is that water frost tends to form on the instruments used to crush the $N D_{3}$ chunks through the sieve, and this frost falls through to the bottom of the sc. To avoid contaminating the subsequent batch of $\mathrm{ND}_{3}$ with water, we 
chose to discard material from the bottom of the sc. The only reason for including the sc as part of the vacuum system was this recycling step which we now consider to be unnecessary.

\section{Irradiation}

Paramagnetic centers are introduced to the target material in two irradiation steps. The first was preformed at liquid argon temperatures $(\approx 84 \mathrm{~K})$ at the Bates electron accelerator. ${ }^{1}$ Using $320 \mathrm{MeV}$ electrons, approximately $10^{18}$ electrons $/ \mathrm{cm}^{2}$ were passed through the target. This caused the previously clear crystals to turn dark purple in color. The second irradiation was done at $1 \mathrm{~K}$ using $800 \mathrm{MeV}$ protons from the LAMPF proton beam. Approximately $0.8 \times 10^{14}$ protons $/ \mathrm{cm}^{2}$ passed through the target material. Though we had hoped to measure the achievable target polarization between the two irradiation steps, so as to monitor the effect of the second, equipment problems made this impossible. Optimal valu es for irradiation have been discussed elsewhere $[\mathrm{BP}][\mathrm{SW}]$.

\footnotetext{
${ }^{1}$ Thanks to D. Crabb and associates for their expertise and use of their cryostat.
} 


\section{Bibliography}

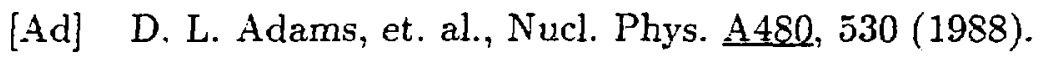

[AG] A. Abragam and M. Goldman, "Nuclear Magnetism: Order and Disorder", (Clarendon Press, Oxford, 1982), chapter 6.

[AS] Milton Abramowitz and Irene A. Stegun, "Handbook of Mathematical Functions with Formulas, Graphs and Mathematical Tables", National Bureau of Standards Applied Mathematics Series 55, (1972).

[AS-1] See [AS], equation 7.3.33 on page 59 .

[Ab] A. Abragam, "The Principles of Nuclear Magnetism", (Oxford University Press, London, 1961), page 75.

[Ab-1] See [Ab], chapter II.

[BP] K. H. Althoff, et. al., "Proceedings of the 4th International Workshop on Polarized Target Materials and Techniques",W. Meyer Ed., (Bad Honnef, 1984), page 30.

[BR] N. Bloembergen and T. J. Rowland, Acta Met. 1, 731 (1953).

[BI] R. J. Blinstoyle and M. A. Grace, Handbook of Physics 42, 558 (1957). 
[CR] M. H. Cohen and F. Reif, Solid State Plyysics 포, (1957), page 321.

[CR-1] See [CR], page 342 .

[CR-2] See [CR], page 333.

[De] W. de Boer, "Dynamic Orientation of Nuclei at Low Temperatures." CERN 74-11 Laboratory I Nuclear Plyssics Divsion, (1974).

[Do] Rainer Dostert, diplomarbeit, Bonn University, Bonn - IR -86-13. (1986).

[Gu] E. Gülmsz, et. al., to be submitted to Phys. Rev. C.

[Go] Maurice Goldman, "Spin Temperature and Nuclear Magnetic Resonance In Solids," (Oxford University Press, London, 1970).

[Gs] Herbert Goldstein, "Classical Mechanics," (Addison-Wesley, 1950).

[Ha] O. Hamada, et al., Nucl. Inst. Meth. 189, 561 (1981).

[Hi] D. Hill and T. Kasprzyk, Argonne Report Series ANL-HEP-PR-84-32. (Argonne National Laboratory, Argonne, Illinois, 1984).

[IM] IMSL Computer Mathematics Library, Houston, (1989).

[JE] Friedrich Lösch,"Tables of Higher Functions," (McGraw-Hill, 1960).

[Kn] Donald E. Knuth, "The Art of Computer Programming", volume 2. (Addison-Wesley, 1981).

[Li] M. J. Lighthill, "Introduction to Fourier Analysis and Generalized Functions," Cambridge Tniversity Press, (Cambridge, 1978).

[Li-1] See [Li], page 43. 
[Mo] J. J. More, "Numerical Analysis," G. A. Watson, Ed., (SpringerVerlag, New York, 1977).

[No] David E. Norton, Microwave Journal, Nov. 1988, page 117.

[Ra] Simon Ramo, "Fields and Waves in Modern Radio," (Wiley, 1953).

[Ra-1] See [Ra], page 41 .

[Rams] N. F. Ramsey, "Nuclear Moments," (Wiley, New York, 1953), page 7-8 and page 23. Also C. H. Townes and A. L. Schawlow, "Microwave Spectroscopy," (Dover, New York, 1975), page 132.

[SW] J. W. T. Spinks and R. J. Woods, "An Introduction to Radiation Chemistry," (John Wiley and Sons Inc., New York, 1990).

[Sl] C. P. Slichter, "Principles of Magnetic Resonance," second edition, (Springer-Verlag, New York, 1980).

[Sl-1] See [Sl], chapter 9.

[Sp] F. Sperisen, Nucl. Inst. Meth. A260, 455 (1987).

[To] Richard C. Tolman, "The Principles of Statistical Mechanics," (Dover Publications, Inc., 1979), chapter IX.

[To-2] See [To], chapter X.

[WW] E. T. Whittaker and G. N. Watson, "A Course in Modern Analysis," (Cambridge University Press, London, 1973), page 76.

[We] W. Th. Wenckenbach, Proceedings of the End Workshop on Polarized Target Materials, G. R. Court et. al. Eds., (Rutherford, 1980), page 10. 
This report has been reproduced directly from the best available copy.

It is available to DOE and DOE contractors from the Office of Scientific and Technical Information,

P.O. Box 62,

Oak Ridge, TN 37831.

Prices are available from

(615) 576-8401, FTS 626-8401.

It is available to the public from the

Nationsl Technical Information Service,

U.S. Department of Commerce,

S285 Port Royal Rd.,

Springfield, VA 22161. 